\title{
TRANSFORMATIONS TERRITORIALES ET MUTATIONS PRODUCTIVES DANS LA REGION METROPOLITAINE DE BUENOS AIRES.
}

\author{
LES CAS DES MICRO-REGIONS \\ DE ENSENADA-BERISSO ET DE \\ ING.ALLAN-EL PATO-EL PELIGRO
}

Horacio Bozzano

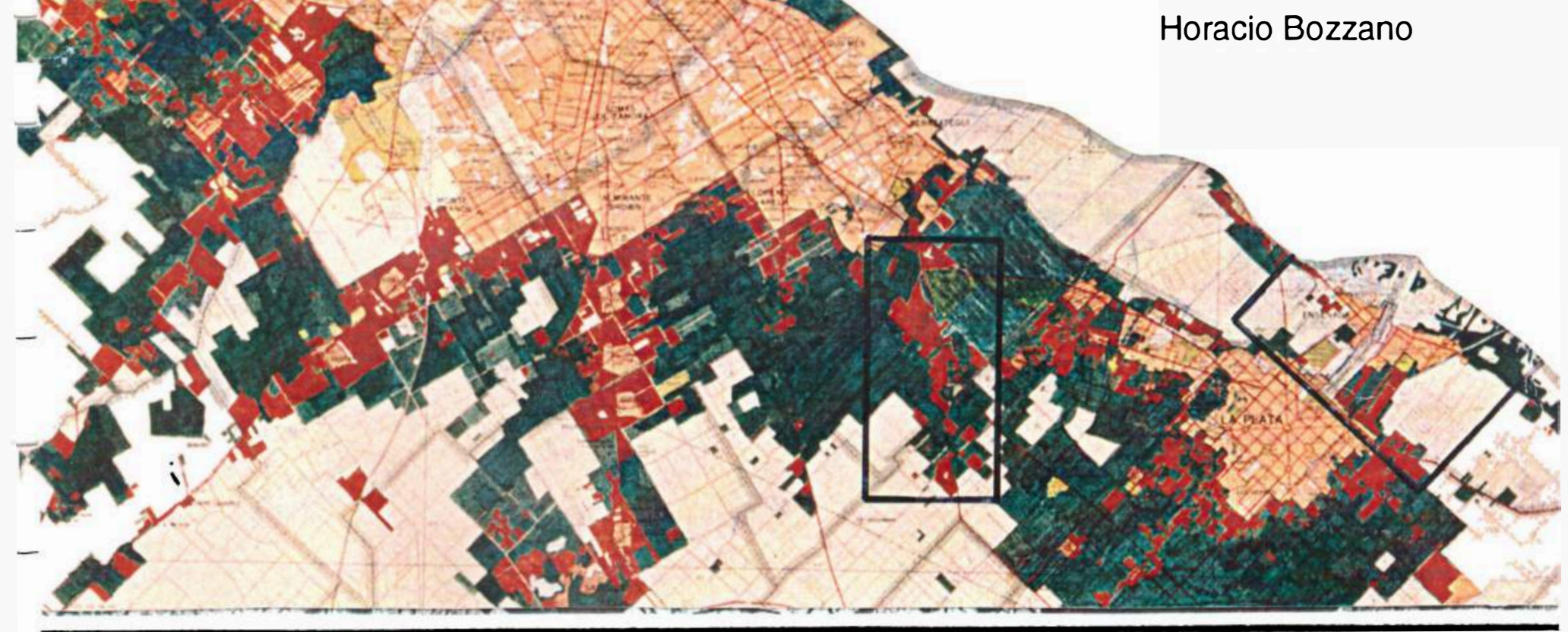

UNIVERSITE DE PARIS III-SORBONNE NOUVELLE

INISTITUT DES HAUTES ETUDES DE L'AMERIQUE LATINE

Paris, Septembre 1992 
DIPLOME D'ETUDES APPROFONDIES

GEOGRAPHIE. AMENAGEMENT. URBANISME

MEMOIRE

\title{
TRANSFORMATIONS TERRITORIALES ET MUTATIONS PRODUCTIVES DANS LA REGION METROPOLITAINE DE BUENOS AIRES
}

\section{LES CAS DES MICRO-REGIONS DE ENSENADA-BERISSO ET DE ING.ALLAN-EL PATO-EL PELIGRO}

\author{
Horacio Bozzano \\ Professeur à l'Université de La Plata \\ Chercheur au CONICET, Argentine \\ Directeur : Dr Jean REVEL-MOUROZ
}

Année 1991-1992 


\section{I - CONCEPTUALISATIONS}

SUR TERRITOIRE ET PRODUCTION

1-Les concepts autour

des transformations territoriales ............................

2-Les concepts autour

des mutations productives

3-Les rapports entre territoire et production.

4-Les transformations territoriales et les mutations productives

\section{II - LA REGION METROPOLITAINE DE BUENOS AIRES........ 15}

1-La petite ville, le port et la production pour le marché local...................... 16

2-La capitale, le pouvoir politique et

la première manufacture de la Pampa.......... 18

3-La ville des immigrants

et les premières grandes industries

4-La ville cosmopolite et la première substitution d'importations........... 25

5-La démarche vers la périphérie et la deuxième substitution d'importations

6-La tertiarisation métropolitaine,

la désindustrialisation

et la reconversion productive

Cartographie

de la Région Métropolitaine. 
Le milieu naturel.............................................................. 54

Transformations territoriales

et mutations productives.

Un essai de périodisation.

1-Les "suertes de estancia" 55

2-Le "Fuerte de Ensenada de Barragán" 56

3-Les "saladeros",

la connexion à Buenos Aires

et la naissance de Berisso....................................... 57

4-Le Port La Plata, l'immigration

européenne et les frigorifiques.......................... 59

5-La Raffineria La Plata de YPF

et la survivance du frigorifique........................... 61

6-La consolidation urbaine

et la diversification industrielle.......................... 63

7-Les Pôles Pétrochimique et Informatique, la Zone Franche du Port La Plata et les "villes-dortoir"
a)Le Pôle Pétrochimique de Ensenada
b)Le Pôle Informatique de Berisso
c)Le Polygone Industriel
d)Le Port La Plata et la Zone Franche
e)Le chantier naval "AFNE"
f)Le Pont Internationale Argentine-Uruguay
g)La déclination des productions intensives
h)La déterioration du milieu

Cartographie et Figures

de la Micro-région. 
Le milieu naturel.................................................... 89

Transformations territoriales

et mutations productives.

Un essai de périodisation

1-Les "Estancias" et les "Tambos" 89

2-L'expansion périurbaine explosive....................... 91

3-L'impact de "Peugeot", l'occupation

des lotissements et l'expansion des

productions intensives.

4-La désindustrialisation,

le sous-emploi et

la mutation productive récente.

a)La décadence de "Peugeot

b)La reconversion de l'industrie automobile

c)Les autres industries dans la micro-région

d)Le sous-emploi et l'appauvrissement des quartiers populaires

e)Les résidences secondaires et l'espace de loisir

f)La crise maraîchère

g)Les mutations aux productions intensives

Cartographie et Figures

de la Micro-Région. 
1 Territoire

et production industrielle................................... 119

2 Territoire

et production agricole intensive.

3 Territoire, production et acteurs. 124

Reflexion finale. 125

BIBLIOGRAPHIE

STASTISTIQUE 


\section{INTRODUCTION}

Les grandes villes sont la scène des transformations territoriales et des mutations productives les plus significatives, par rapport à celles des espaces ruraux, des villes moyennes ou des villages.

La concrétisation des processus sociaux, économiques et politiques divers forme un ensemble d'aspects qui tend à organiser des territoires avec des niveaux d'immutabilité remarquables. Cette "inertie territoriale" se manifeste, dans une première analyse, dans des situations de permanence assez semblables quel que soit l'espace concerné: les métropoles, les villes, les villages ou les espaces agricoles divers.

De la même façon, l'introduction de nouvelles technologies de production dans des périodes variées donne lieu à des mutations peu perceptibles à court terme à l'échelle globale. Les changements à l'intérieur des processus productifs émergent dans la société le plus souvent sous la forme de conflits des travailleurs ou de syndicalistes, assez ponctuels. Les mutations dans la production conduisent aussi à altérer les rapports de pouvoir établis entre des producteurs, des travailleurs et l'Etat.

Comment se produisent des transformations territoriales dans une grande ville ? Où les manifestations les plus visibles ont-elles lieu ? Dans quelle mesure les mutations productives interviennent-elles? Existe-il des relations directes entre ces deux processus, ou est-il possible de définir une articulation avec des médiations d'une autre nature ? Quel degré de généralisation conceptuel pourrait-on atteindre à partir des cas de recherche?

Ce travail s'inscrit dans cette perspective générale. Il essaie de constituer un apport orienté à la réponse à ces questions, en ce qui concerne la région métropolitaine de Buenos Aires, et plus particulièrement $\mathrm{sa}$ périphérie.

La sélection des territoires d'étude a un rapport étroit avec les deux objets de recherche: les transformations territoriales et les mutations productives. Les correspondances et les décalages sont suffisamment larges et hétérogènes pour justifier un sujet propre de 
recherche. Il s'agit de scénarios où se produisent des mutations en systèmes productifs totalement différents. Le but est précisémment de couvrir une gamme de types de production assez ample pour permette de répondre aux hypothèses de travail.

A partir de niveaux d'approximation selon deux échelles, les territoires d'étude sont les suivants (FIGURE 1):

a) Région Métropolitaine de Buenos Aires.(1580-1992) En résumé: Port. Centre commercial. Manufactures. Localités et quartiers.Ceinture maraîchère. Industrialisation. Stations ferroviaires et sous-centres. Suburbanisation. Métropolisation.

b) Micro-région de Ensenada-Berisso. (1618-1992) En résumé: Elevage. Forteresse. Centres commerciaux et résidentiels. Manufactures. Port. Frigorifiques. Horticulture. Raffinerie. Chantiers navals. Sidérurgie. Pôles Pétrochimique et Informatique.

c) Micro-région de Ingeniero Allan-El Pato-El Peligro (1800-1992). En résumé: Elevage. Exploitations laitières. Grands lotissements. Industrie textile. Usine automotrice. Horticulture et floriculture. Petite et moyenne industrie. Résidence secondaire.

Les objectifs généraux de la recherche sont:

a) Analyser les processus d'organisation territoriale des cas d'étude, en mettant l'accent sur les périodes actuelles.

b) Identifier les transformations territoriales et les mutations productives les plus significatives dans la conformation des territoires d'étude.

c) Reconnaître les articulations et les décalages entre ces transformations territoriales et ces mutations productives.

d) Etablir des conceptualisations autour des rapports territoire-production industrielle, territoire-productions intensives et territoire-production-acteurs. 
Dans le dessein de la recherche, les hypothèses se dirigent vers l'explication de relations de caractère global entre des transformations territoriales et des mutations productives.

1 Les mutations industrielles dans la région métropolitaine sont un des processus déterminants des transformations territoriales.

2 Les mutations dans la production agricole sont le facteur principal de différentiation territoriale entre la périphérie métropolitaine et les systèmes agraires traditionnels.

3 Les mutations productives et l'innovation technologique tendent à produire des majeurs décalages sociaux et économiques entre les acteurs directement concernés et ceux qui ne reçoivent pas le changement. 


\section{FIGURE 1}

\section{REGION METROPOLITAINE DE BUENOS AIRES}

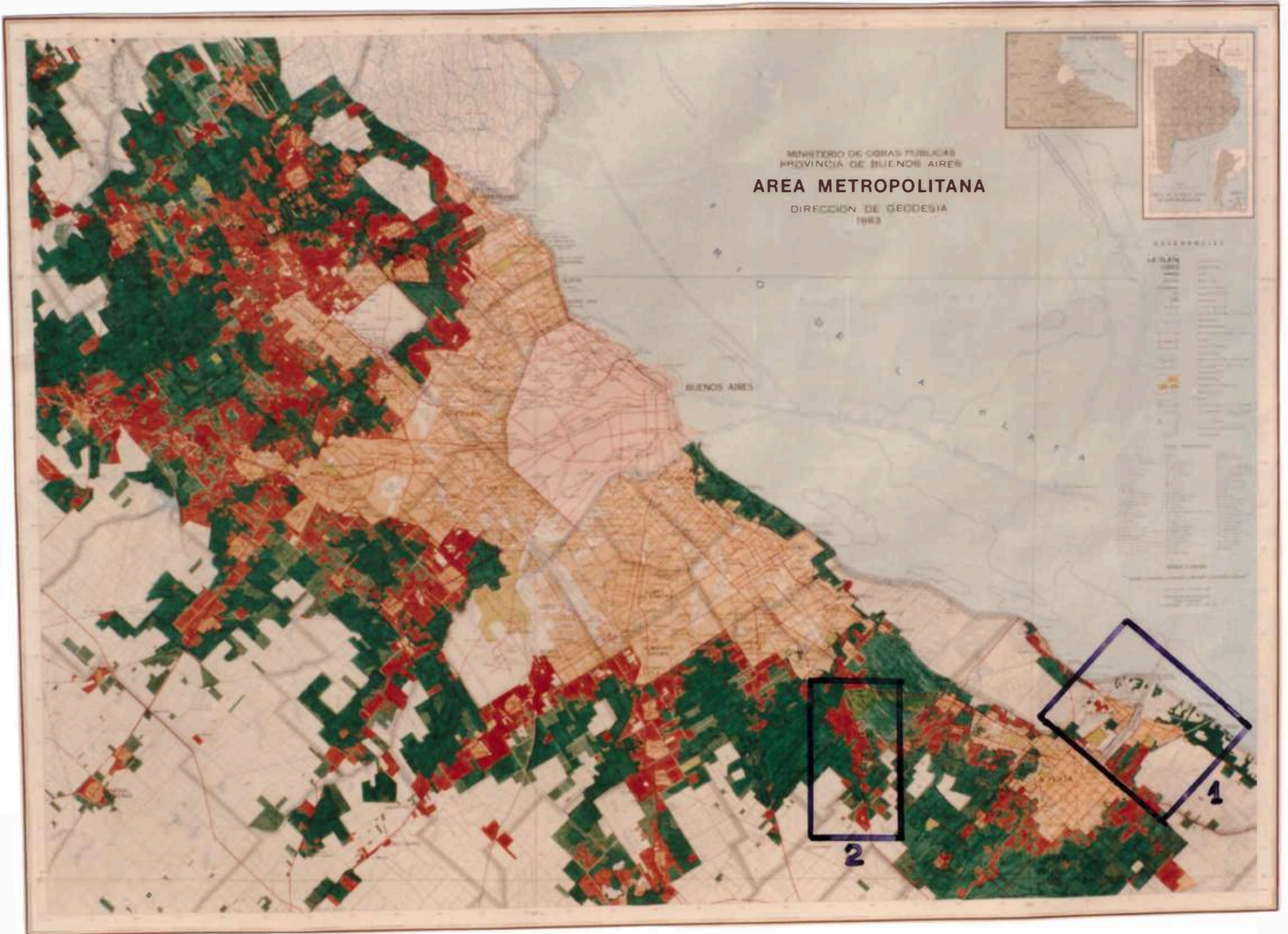

1-MICRO-REGION DE ENSENADA-BERISSO 2-MICRO-REGION DE INGENIERO ALLAN-EL PATO-EL PELIGRO

Les espaces périurbains sont représentées en couleurs rouge et vert, pour indiquer respectivement les parcelles urbaines et rurales. Carte realisée par H.Bozzano, P.Pintos et C.Bevilacqua.

Source: Carte "Area Metropolitana", Dirección de Geodesia, Provincia de Buenos Aires, 1983. Echelle 1:100.000 


\section{I - CONCEPTUALISATIONS \\ SUR TERRITOIRE ET PRODUCTION}

\section{I.1 LES CONCEPTS \\ AUTOUR DES TRANSFORMATIONS TERRITORIALES}

Le concept de "territoire" suscite normalement de nombreuses acceptions qui varient selon la discipline scientifique et les orientations des divers courants théoriques.

En ce qui concerne la Géographie, il existe une affinité conceptuelle assez remarquable entre les notions d'"espace" et de "territoire" Cela ne signifie nullement une similitude parfaite entre ces deux concepts, mais plutôt un rapprochement itératif à plusieurs moments au long de l'évolution de la connaissance géographique.

A partir des années soixante, un développement significatif existe autour de cette question. Avant cette période on remarque des notions de "paysage", comme reflets d'espaces, et d'"espace homogène" de J.Boudeville: un espace continu, dont chaque partie présente des caractéristiques aussi proches que celles de l'ensemble.

L'évolution conceptuelle d'"espace homogène" à celui d'"espace fonctionnel" (Dollfus;1976); même celui d'"espace relatif" à "espace relationnel" (Harvey;1983) sont des efforts analytiques qui contribuent à éclaircir cette question.

"Il n'y a aucune nécessité d'adopter une perspective rigide face au propre concept spatial, soit avec des fins philosophiques, soit pour recherche empirique. Le propre concept peut être considéré comme flexible étant défini dans des contextes déterminés, en récevant divers symbolismes et étant formalisé en divers langages spatiaux." (Harvey; 1983:240)

L'"espace en tant que "...support de la vie et de l'activité, (..qui constitue un) obstacle à la vie de relations (...et qui est la) base de l'activité symbolique" (Raffestin et Turco;1984) permet d'introduire l'analyse des processus naturels et sociaux qui acquièrent une dimension spatiale pour se déployer. 
La procédure pertinente pour une étude rigoureuse, en suivant Coraggio (1987), était de commencer par le rapport de la catégorie "espace" avec des processus naturels, de continuer en introduisant cette catégorie dans le rapport nature-société et d'identifier finalement la relation de cette construction avec l'espace idéal des géométries.

Mais, par rapport à l'"espace", le "territoire" est postérieur. Il est "...produit à partir de l'espace par les réseaux, les circuits et les flux projetés par les groupes sociaux" (Raffestin; op.cit). La plupart de ces réseaux, circuits et flux répondent à des mécanismes qui suivent certaines rationalités sociales. Cependant ils ont d'abord répondu, dans une moindre mesure, à des rationalités propres à l'ordre naturel. Cela ne signifie pas une séparation entre le social et le naturel, mais une "surconstruction" de l'un par rapport à l'autre.

Quel est le degré de permanence d'un territoire ? Par quels aspects est-il possible d'identifier les changements ? Sont-ils perceptibles dans le court terme ? La notion de "transformation territoriale" ne suppose pas un changement total et abrupt des composants de l'espace, mais un ensemble de faits dynamiques qui s'articulent selon des liens complexes, pendant des durées variables, pour produire des modifications significatives à moyen terme.

L'implantation d'une nouvelle branche industrielle, la réalisation d'une grande oeuvre d'infrastructure urbaine, l'établissement d'un "quartier spontané", les effets de l'application d'une régulation des transports ou d'une loi de contrôle d'usage du sol, révèlent bien des situations dynamiques de durée variable qui peuvent survenir dans un même endroit. Malgré leur caractère asynchrone, elles s'enchaînent d'une manière synergique pour occasionner des transformations dans le territoire.

Dans quelle mesure interviennent les mutations productives? Contribuent-elles par elles-mêmes à modifier et à renouveler des territoires ou s'insèrent-elles dans d'autres processus, plus amples ?

\section{I.2 LES CONCEPTS AUTOUR DES MUTATIONS PRODUCTIVES}

Le concept de "production" qui concerne cette recherche provient de l'Economie. Il s'agit d'un de ceux qui permettent, avec la distribution et la consommation, d'étudier les richesses matérielles dans une société. 
En tant qu'objet pour la Géographie et pour l'Aménagement, l'étude de la production acquiert une signification dans la mesure où elle s'inscrit dans les analyses territoriales, quelle que soit l'échelle à laquelle on l'aborde.

L'acception sélectionnée inclut comme objet de traitement les biens créés par l'agriculture et par l'industrie, au sens large. De toutes manières, il faut souligner l'importance d'une tendance à relier la "production de services" -et de connaissance- avec la production proprement dite. Même si la "tertiarisation" intègre de plus en plus de petits cycles de production industrielle dans son processus de complexisation, il est possible de différencier encore, à l'intérieur, les services mêmes de la production.

Que comprend la notion de "mutation productive" ? Exprime -t-elle toujours une mutation technologique? Quelles en sont les manifestations les plus saillantes ? La notion de "mutation productive" représente soit la conversion, soit l'introduction d'une nouvelle activité productive. Cela peut être provoqué par la mutation technologique proprement dite mais pas toujours.

En ce qui concerne l'innovation technologique dans la production, il faut la différencier de son processus de diffusion. Il y a un "...décalage entre découverte et application économique" (Ait-El-Hadj;1985a), qui se manifeste davantage dans le cas des pays non-industrialisés ou de ceux dits d'"industrialisation tardive" A cette situation s'ajoute celle de la confrontation entre "...savoir concret de production et savoir d'étude et de conception".(Marger;1985)

Les trois grandes transformations du "système technique" (Gille; 1978) auront lieu dans des régions relativement réduites. La plupart du monde a été le scénario de réception, de diffusion et de commercialisation des innovations, très souvent avec des décalages temporels assez considérables.

Dans ce cadre, l'étude des mutations productives dans des pays d'"industrialisation tardive", n'incorpore pas cette question comme un déterminant central. Il s'agit de sociétés qui, tout au long de leur histoire, n'auront pas été porteuses d'innovations: il n'est pas impossible qu'elles le soient maintenant ou dans le futur. L'objet de l'étude concerne particulièrement l'introduction ou la conversion des technologies de production appliquées déjà dans d'autres endroits. En ce sens il est possible d'accepter le changement comme "mutation technologique" 
Cette recherche a, entre autres objectifs, celui d'éclairer la question sur les décalages temporels entre l'innovation et leur diffusion, différences qui s'accentuent selon le niveau d'industrialisation de chaque pays.

\section{I.3 LES RAPPORTS ENTRE TERRITOIRE ET PRODUCTION}

Traditionnellement, l'industrie se rattache à la ville et l'agriculture à la campagne, mais une analyse plus fine peut conduire à trouver des exceptions dans ce type de rapport.

Il y a des fabriques implantées en milieu rural, soit du fait de la connexion étroite entre les matières premières et l'industrialisation ultérieure dans un cycle de production, soit du fait de localisations pionnières des grandes usines liées à certains régimes de promotion de l'Etat. Sans prendre systématiquement cette forme, la logique d'installation industrielle est majoritairement urbaine à cause des "effets utiles d'agglomération" (Topalov;1979). Mais dans les métropoles il y a aussi des logiques de localisation différentes liées à plusieurs facteurs, entre autres la disponibilité en terre, la fourniture de services, l'accessibilité intra-urbaine, les conditions environnementales et la date d'installation par rapport au processus d'organisation territoriale urbaine.

En ce qui concerne l'agriculture il est posssible de trouver des espaces maraîchers dans des interstices urbains ou très souvent des couronnes périurbaines, pas toujours continues, avec des exploitations qui pratiquent des activités agricole-intensives diverses, notamment de type agro-industriel. Le maraîchage, la floriculture, la culture en pépinière, l'aviculture et certaines fermes, sont des productions étroitement liées à des marchés de consommation urbaine.

Même si le développement technologique en matière de transport, de réfrigération ou d'autres formes de conservation des produits, tend à relativiser la localisation de ces activités à proximité de la ville, il y a d'autres facteurs très significatifs qui contribuent à maintenir ce genre de rapport territoire-production. Il s'agit de la relation entre la rentabilité des productions agricoles et la variation des rentes différencielles urbaines et agraires dans la zone périurbaine.

Territoire et production: leur analyse peut conduire à identifier des "espaces productifs" dans une ville ou dans une aire rurale, et plus encore, à établir des liaisons avec des "espaces de distribution" ou "de 
consommation" Mais, la réflexion sur les transformations territoriales et les mutations productives suppose une instance de recherche postérieure à cette analyse.

\section{I.4 LES TRANSFORMATIONS TERRITORIALES ET LES MUTATIONS PRODUCTIVES}

Si à travers l'étude des rapports "territoire-production" il est possible d'identifier des manifestations, des émergences d'un certain processus de formation d'un espace, aborder les relations entre des transformations territoriales et des mutations productives conduit à reconnaître ses déterminants, l'ensemble des causes qui donnent lieu à la génèse du même territoire.

Pourquoi interviennent-ils comme déterminants ? Parce que quel que soient la nature et le degré des transformations et des mutations, ils représentent les moteurs du changement. Cela ne signifie pas une exclusivité dans ce rapport. Il y a aussi d'autres déterminants "nonproductifs": il s'agit des processus sociaux particuliers, des facteurs politiques ou même des explications de certaines pratiques culturelles.

La localisation d'un grand établissement industriel attire un nombre considérable d'employés et souvent de petites industries dépendantes de son cycle productif. Cette situation déchaîne la croissance des activités commerciales et de construction. La mobilisation de composants hétérogènes de la ville, brièvement décrite à partir de l'industrie n'est pas la caractéristique dominante actuelle.

La reconversion industrielle récente signifie généralement un changement à l'intérieur du processus de production en termes de flexibilité et d'automatisation. La "productique" entraîne de nombreuses conséquences: en ce qui concerne cet objet, il s'agit particulièrement de la spécialisation et la réduction d'emploi et, dans certains cas, de la refonctionnalisation des espaces industriels. Les effets de cette situation sont assez différents selon qu'il s'agisse de sociétés économiquement développées ou de sociétés non-développées.

L'épanouissement de nouvelles exploitations maraîchères est en relation avec la croissance globale du marché de consommation urbaine. Cette situation ne déchaîne pas la prolifération de nombreuses activités connexes, sauf le déployement de circuits économiques propres: la 
consolidation de certains réseaux de distribution et de commercialisation urbaines. L'influence de cette activité comme productrice d'emplois n'a pas la même importance que dans l'industrie.

Les changements à l'intérieur des exploitations agricoles propres de la périphérie métropolitaine ne charrient pas une innovation technologique déterminante pour sa mutation productive. Celle-ci est principalement dûe à d'autres facteurs liés à l'insertion dans des circuits économiques, à la taille des exploitations et à l'introduction d'"agro-chimiques"

L'ampleur des mutations productives est donnée, non seulement par la diversité d'activités agricoles et industrielles, mais par l'hétérogénéité des transformations techniques dans de nombreuses branches de biens, et même, en certain cas dans chaque produit.

En ce qui concerne le rapport "transformations territoriales-mutations productives", l'analyse des changements à l'intérieur des processus de production en eux-mêmes ne constituent pas les déchaînements directs des transformations du territoire. L'objectif est précisément d'identifier dans quelle mesure et à travers quelles médiations les unes peuvent contribuer à expliquer les autres. 


\section{II - LA REGION METROPOLITAINE DE BUENOS AIRES}

L'origine de Buenos Aires n'est pas liée à la production, mais à des facteurs stratégiques et même à des fonctions commerciales et portuaires. La position périphérique par rapport à des schémas territoriaux et à l'exploitation de ressources par la Couronne d'Espagne est le principal facteur qui explique cette situation.

La subsistance du village portuaire pendant presque deux siècles est relié directement à des activités commerciales et de contrebande. Son isolement et son éloignement constituent les principaux déterminants du développement local des activités productives primaires orientées vers la consommation locale.

La période de consolidation et de centralisation du pouvoir à partir de la création de la Vice-Royauté du Río de la Plata est aussi celui du développement d'activités productives particulières.

Cela signifie le début de la manufacture. Un début lié à la valorisation du territoire de la "pampa": il s'agit de l'élevage du gros bétail. Le "saladero", établissement destiné à la salaison des viandes et des cuirs, se situe aux environs de Buenos Aires.

Les mutations productives postérieures concernent un changement considérable dans la manufacture de la viande. Il s'agit de l'introduction de la technologie de conservation par le froid: le "frigorifique" Au-delà de ce processus, il y a des transformations territoriales importantes dans la structure urbaine mais aussi à l'échelle locale.

La diversification ultérieure des activités productives urbaines complexifie notamment l'organisation métropolitaine. A partir de cette période, l'explosion de la croissance démographique et économique ne permet pas d'entrevoir facilement les rapports spécifiques entre des transformations territoriales et des mutations productives, et moins encore des déterminantes particulières. 
Les dernières années enregistrent des mutations productives vers la desindustrialisation et récemment, mais à moindre échelle, vers la reconversion technologique dans l'industrie. Les effets de ce processus dans le territoire sont encore difficiles à évaluer.

Le rapport analysé est le point de départ théorique pour la réalisation d'une périodisation suivant les mutations productives les plus saillantes. Il est important de remarquer que celles-ci n'interviennent pas toujours comme des déterminants des transformations territoriales. Pendant une longue période, la production joue un rôle accessoire dans la formation de l'espace urbain.

\section{La petite ville, le port et la production pour le marché local.}

L'origine même de la ville suppose un aménagement des espaces residentiels et des espaces productifs qui comporte une rationnalité tout à fait intéressante, à tel point qu'aujourd'hui il est possible de vérifier, malgré la profondeur des changements, les vestiges de la première assignation de terres faite en 1580 par Don Juan de Garay, le fondateur de Buenos Aires.(FIGURE 2-page 36 )

Même si la lente conversion de village en ville se traduit en changements territoriaux et en une tendance de diversification productive assez faible pendant presque deux siècles, les lignes du développement sont semblables et permanentes. Jusqu'au contexte où se produisent les conditions générales pour la création de la Vice-Royauté en 1778, la logique de fonctionnement est essentiellement celle d'un port stratégique et de contrebande et d'une ville commerciale, surtout fermée sur elle-même.

En quoi consiste le territoire aménagé ? Le plan de Garay est un tissu en damier en face du Río de la Plata, comprenant 135 ou 144 îlots, dits "manzanas", avec des grands lots, généralement de $3600 \mathrm{~m} 2$. Vers la rivière, il y a trois zones contiguës étroites, dont deux sont destinées à l'horticulture et une au port, mais qui ne sont pas utilisées à un tel but.

Le reste de l'organisation du territoire suit une disposition parallèle à l'axe du Paraná-Plata. (FIGURE 2) Au sud-est il s'agit d'un axe continu de $100 \mathrm{~km}$ de long et $8 \mathrm{~km}$ de large constitué de 29 donations de terre pour l'exploitation d'élevage appelées "suertes de estancia" Vers le nord-ouest, se conformant à la meilleure aptitude naturelle, Garay assigne une frange de $21 \mathrm{~km}$ sur $5 \mathrm{~km}$ pour la production agricole, en incluant 65 exploitations dites "chacras" nommées comme les "tierras de pan llevar" Plus loin il octroie d'autres "suertes de estancia", mais sa 
répartition n'est pas du tout connue; il s'agit d'à peu près 36 donations partagées en trois aires d'élevage. (Figueira; 1983a)

Quel rapport "territoire-production" existe-t-il au début de Buenos Aires ? Il est fondamentalement très étroit à cause du développement difficile et solitaire dans les premières décennies. Il faut ajouter que plusieurs voisins proposent de "re-situer" le village. Dans ce cadre d'isolement presque funeste, la nécessité de subsistance contribue à déployer une gamme d'activités productives parmi tous les habitants.

La plupart des voisins ont des arbres fruitiers, des basse-cours et un terrain maraîcher dans leur propre maison, et plus encore, quelques-uns dans les îlots périphériques inoccupés du plan de fondation. On estime à cette époque-là une superficie cultivée de 500 hectares. Contrairement à une idée assez répandue, la rare population indienne de la "pampa" n'a aucune attitude déprédatoire en vers les cultures et encore moins, belliqueuse. Cependant, les habitants de Buenos Aires doivent être attentifs aux bétails, et aux incursions sporadiques de corsaires par le Río de la Plata.

Les autres terres assignées à l'agriculture et á l'élevage sont l'objet d'une incorporation plus tardive à la production. Le début des exploitations très extensives date d'environ un demi-siècle après la fondation de Buenos Aires, en profitant des vaches et des chevaux redevenus sauvages depuis leur introduction par Pedro de Mendoza en 1536.

La plupart des produits manufacturés, étrangers pendant une longue période, souvent sont l'objet d'un échange illicite par l'argent de Potosí. Les premières périodes de production artisanale proprement dite sont des temps de négociation et de confrontation avec le pouvoir local pour avoir un peu plus de protection. La concurrence inégale des produits manufacturés de l'Europe et des économies régionales de Cuyo, Córdoba et Tucumán, témoigne de la dureté des premières époques à Buenos Aires, ainsi qu'un jeu différent à l'échelle locale des productions agricoles et manufacturées par rapport au territoire.

Le nombre réduit d'habitants est aussi l'autre facteur qui contribue à limiter les possibilités de développement industriel. Pendant plus d'un siècle la taille de Buenos Aires n'est pas suffisante pour promouvoir une expansion manufacturière propre. En bref, l'évolution de la population est la suivante: 300 habitants en 1580,500 en 1602,2070 en 1639, 3360 en 1658,5100 en 1680,8900 en 1720 et 11200 en 1744. 
Si la ville a besoin de la production agricole pour sa subsistence, son contexte ne permet pas le développement des productions artisanales et des manufactures. Soit à cause du territoire même, soit à cause de l'isolement, Buenos Aires est attachée dans presque la moitié de sa vie plutôt à la production primaire qu'à la production secondaire.

\section{La capitale, le pouvoir politique \\ et la première manufacture de la Pampa.}

A partir du dernier tiers du XVIIIème siècle Buenos Aires est le scénario de changements variés concernant la composition de la population et le rythme de croissance, la pression pour la libéralisation de l'activité commerciale, la consolidation de l'exploitation de l'élevage, la naissance de la première industrie de manufacture, et finalement, la constitution d'un groupe très fort de pouvoir politique.

L'origine de la Vice-Royauté du Rio de la Plata en 1776 représente le début d'un processus de concentration du pouvoir politique, économique et culturel à Buenos Aires qui n'a pas cessé jusqu'aujourd'hui. Mais, comment était le territoire à cette période-là ? Les bouleversements politiques et institutionnels étaient-ils reliés aux mutations productives ? L'introduction de nouvelles formes de production: provoquait-elle des transformations territoriales?

Pendant la décennie de 1760 la ville dépasse les 20.000 personnes, la plupart habitant au centre du plan originel de la fondation. Trois places commerciales périphériques au noyau historique, vers le sud, l'ouest et le nord sont créées. Elles constitueront plus tard, les trois centres principaux de communication de la métropole. Il s'agit respectivement des actuelles Plaza Constitución, Miserere et Retiro.(FIGURE 3-page 37)

Dans les trois dernières décennies du XVIIIème siècle on définit un réseau territorial avec d'autres petits centres et une diversité significative d'activités dans des espaces intermédiaires. Il s'agit de productions agricoles, d'espaces pour le loisir et la récréation et peu après, d'une production de manufacture naissante.(FIGURE 3)

Les trois axes de communication principaux vers l'intérieur relient à travers des chemins de charrettes, dits "caminos de carretas", une série de petits hameaux et de postes de chevaux dits "postas" Il y a une relation avec la production, soit de service et d'approvissionnement par le transport de marchandises depuis l'ouest jusqu'au nord lointain, soit de petites productions locales pour le marché de consommation en croissance de Buenos Aires. 
Malgré l'importance traditionnelle des routes coloniales vers le HautPérou et le Chili, le développement urbain dans l'organisation de l'espace de Buenos Aires proprement dit suit d'abord une autre orientation.

Le manque d'un port pendant trois siècles sur le Río de la Plata, les conditions d'amarrage naturel dans le Riachuelo et la valorisation du territoire pour l'élevage au sud-est de la "pampa", contribuent à des échelles différentes à faire naître lentement les premiers espaces urbains et industriels vers le sud. (FIGURE 3)

La traversée du Riachuelo, qui se faisait en canots à partir de 1653, necessitera la construction d'un pont. Les essais, comencés en 1750, finissent avec l'inauguration du "Puente de Galvez", 41 ans après. A cette époque-là, il existait le hameau de Barracas, une baraque née en 1731 à cause du déplacement d'un contingent d'esclaves de la "Banda Oriental del Uruguay" (Figueira; 1983a) Sa croissance est également liée aux fours à briques et à tuiles et à de vieux moulins à blé, dits "tahonas"

Le chemin entre Buenos Aires et Barracas, parmi d'autres vers le nord et l'ouest, est jalonné de "chacras" des exploitations fruitières, maraîchères et céréalières de moins de 25 hectares, aussi bien que quelques proprietés priveés pour le loisir. (FIGURE 3)

La fin du XVIIIème siècle marque le début de San José de Flores, actuel quartier de l'ouest de la ville. En s'opposant à Barracas, il est aménagé en 1804: une place, une chapelle, 80 îlots et des terrains destiné à l'abattoir. La première moitié du XIXème est celui du développement des maisons d'été dit "quintas de veraneo" entre Buenos Aires et Flores.

L'origine de Belgrano comme village aménagé, vers le nord, date de 1855, mais cet endroit était un poste de charrettes il y a presque deux siècles. Depuis 1726 fonctionnait "La Calera", gisement de sable et de coquillier avec un four à chaux (Figueira; 1983a)

Plus éloignées, en toutes directions, d'autres villages complétent le réseau territorial mais avec un moindre degré de connexion. il s'agit de San Isidro, San Fernando et le petit port de cabotage de Las Conchas vers le nord; San Martín, Morón, Merlo y Moreno, vers l'ouest; et Quilmes, La Paz (Lomas de Zamora), San Vicente et le Port de la Ensenada de Barragán vers le sud.

C'est précisemment la Ensenada de Barragán l'endroit où s'établit en 1798 le premier "saladero" constitué d'un abattoir et d'installations assez 
rudimentaires pour l'extraction du suif, pour saler et sécher la viande en bandes dit "charqui" ou "tasajo", et pour préparer le cuir à l'exportation. Une vingtaine d'établissements jusqu'en 1820, situés pour la plupart à Barracas ou ses environs, suivront celui-là.

Entre 1770 et 1820 ont lieu, donc, plusieurs transformations territoriales et des mutations productives significatives. On définit une configuration spatiale particulière: un centre consolidé avec trois places commerciales périphériques, une couronne adjacente de "chacras" d'usage agricole intensif, trois villages et des postes de charrettes. Pendant cette cinquantaine d'années le rythme de croissance de la population est supérieur à ceux de Lima, de La Havane ou de México, passant de 22.000 habitants en 1770 à 32.000 en $1790,39.000$ en 1800 , 45.000 en 1810 et 55.400 en 1822 .

La valorisation des ressources provenant de l'élevage et des activités propres à la"pampa" que son développement suscite, représentent une mutation productive de caractère décisif dans la consolidation du pouvoir politique. A partir de la "Revolución de Mayo" de 1810, les propriétaires des grandes exploitations d'élevage dits "estancieros" deviennent pour la plupart des politiques, des militaires et des bureaucrates détenant le pouvoir.

Buenos Aires et ses environs concentrent les établissements manufacturiers nécessaires à l'exportation croissante de viande salée, de cuir, et plus tard, de laine de mouton. Dans la première moitié du XIXéme siècle, 70 à $85 \%$ des exportations argentines provenaient des produits semi-manufacturés (presque primaires) de l'élevage de la "pampa" Dans ce cadre les "saladeros" sont les plus importants, situation renforcée par la protection de l'Etat: cette industrie contribuait à moins de $1 \%$ de la rentrée d'impôts.

Barracas et Barracas-al-Sud, après Avellaneda, forment l'origine du premier territoire urbain-industriel de l'agglomération. Le scénario est un espace à vocation portuaire depuis le début de la ville de Garay, renforcé par l'établissement de baraques d'esclaves et par d'autres activités: les fours à briques et une "tahona", vieux moulin à blé. Entre 1810 et 1855 les "saladeros" sont de plus en plus importants. Les "travailleurs devenus urbains" dans cette activité reproduisent des formes de vie rurale. Les logements avec des toits de joncs et cannes, les "pulperías" et les "boliches" sortes de bazar et des débit de boissons, sont l'habitat du journalier dit "peón de campo" et même des vagabonds connus comme "vagos y mal entretenidos" Vers 1850 on estime à plus de 25.000 les "peones", les tueurs d'animaux dits "matarifes", les bouchers, les muletiers de bétails dits "arrieros" et les "vagos", installés 
pour la plupart aux environs des "saladeros" de Barracas. La ville qui se développe est une sorte de prolongation de la campagne à Buenos Aires.

Mais la première activité de manufacture importante de la "pampa" développée tout au long du XIXème siècle ne signifie pas une mutation technologique proprement dite. Le principal instrument de travail du "saladero" était le couteau. D'après une perspective industrieuse et assez partielle, le consul anglais informait en 1850 qui "...un moulin à vapeur pour moudre le blé et une douzaine de baquets à vapeur pour extraire la graisse des ossements sont les seules pièces de machinerie (à Buenos Aires), et elles ont toutes été fabriquées en Grande-Bretagne." (cité dans: Lynch;1983)

Malgré le retard technologique de l'industrie manufacturière, le développement de l'industrie artisanale est significatif. Dans la décennie de 1830, Buenos Aires compte des établissements de tannerie, de charpenterie, de forge et d'argenterie; et plusieurs autres activités. Une proportion considérable d'entre eux est attachée aux activités belliqueuses et de défense de la période de Rosas.

La seconde moitié du XIXème siècle constitue le scénario des fortes transformations territoriales plus en rapport avec l'introduction de technologies, assez récentes en matière de transport et d'autres services urbains, qu'à l'introduction de nouvelles technologies de production. Cette sorte de décalage entre "territoire-technologie de services" et "territoire-technologie de production" sera central dans la formation de Buenos Aires comme région métropolitaine proprement dite.

Entre 1850 et 1880 il y a un développement solide de divers services. Il s'agit des moyens de transport, de l'éclairage public, de l'eau courante, des égouts, des hôpitaux, des cimetières et des promenades de loisir.

En ce qui concerne le transport, on passe du cheval et du char à l'omnibus à cheval en 1853 et au tramway à cheval en 1865. Le réseau ferroviaire introduit en 1857, atteint $149 \mathrm{~km}$ vers 1890 , desservant un vaste territoire. Entre 1853 et 1878 on crée quatre entreprises d'éclairage appellées "Compañías de Gas" qui installent environ 8000 lampions. Parmi les nombreux espaces de loisir, on peut remarquer les places, les clubs et les théâtres du centre-ville et des promenades diverses vers les quartiers du nord (Sarrailh;1983)

Les épidémies de choléra de 1867 et de fièvre jaune de 1871 contribuent à accélérer les investissements en services de santé et de salubrité commencés en 1859 avec le projet intégral des eaux et des 
égoûts. Vers 1880 le réseau d'eau courante approvisionnait un quart de la population. Le service de ramassage d'ordures date de 1875. Les oeuvres des égoûts s'intensifient entre 1873 et 1887. (Sarrailh;1983)

Entre 1842 et 1880 on installe douze grandes hôpitaux. En 1871 est mise en oeuvre la première section du plus grand cimetière de Buenos Aires: la Chacarita. (Sarrailh; 1983) Les deux dernières épidémies avaient occasionné environ 30.000 décès: une proportion très haute pour une population qui de 76.000 habitants en 1852 avait dépassé les 150.000 en 1869.

Les problèmes croissants de salubrité des "saladeros" qui seront incorporés à la ville par son expansion, donnent lieu à une importante relocalisation intensifiée depuis 1861. Dix ans après seulement restaient trois établissements dans son endroit initial.

L'éloignement des "saladeros" de Buenos Aires et leur dispersion vers la rivière du Plata dans un trajet de plus de $130 \mathrm{~km}$ entre Atalaya (Magdalena) au sud et San Fernando au nord, répresente une transformation territoriale importante.

C'est le début d'une ville avec une claire vocation urbaine: le développement des technologies de services et la rélocalisation d'une industrie avec une culture rurale derrière, en sont les deux témoins principaux. C'est aussi l'époque de définition d'une configuration territoriale pas encore métropolitaine, mais d'un réseau avec un centreville plus "urbanisé" et une périphérie plus productive, concernant des sous-centres avec des vocations industrielles qui émergent et des grands espaces intermédiaires avec des terrains pour le loisir et les exploitations maraîchères.

Un habitant de Buenos Aires de 1850 aurait-il pu concevoir le scénario où il devait vivre trente ans plus tard ? Si les transformations territoriales produites par des innovations technologiques en services et par la consolidation productive des espaces périphériques sont rémarquables, personne n'aurait pu imaginer en 1880 les changements qui devaient avoir lieu vingt ou trente ans plus tard. On assistera, en effet, à une véritable révolution urbaine en ce qui concerne le peuplement et l'industrialisation.

\section{La ville des immigrants} et les premières grandes industries 
A partir de la décennie de 1880 l'Argentine se caractérise par l'intensification des courants migratoires européens, par la consolidation dans la "pampa" d'un modèle "agro-exportateur" puissant et par une mutation productive considérable signée par l'introduction des premières grandes usines industrielles. Ce processus continuera avec certaines variations pendant un demi-siècle.

Malgré les riches et vastes territoires argentins dépeuplés, Buenos Aires c'est le scénario d'une forte concentration démographique, d'une accumulation économique remarquable et d'un renforcement accéléré du pouvoir politique. Mais devant le "bouleversement urbain" généralisé que ces processus contribuent à développer, quel territoire occupe-t-on pour se déployer ? Les mutations industrielles sont-elles des déterminants directs des changements dans l'organisation urbaine ? Quelles sont les transformations territoriales les plus saillantes?

Le territoire de Buenos Aires vers 1880 a la configuration spatiale d'une ville proprement dite très valorisée par l'introduction de services, avec des sous-centres en processus de spécialisation fonctionnelle et un espace agricole à usage intensif de plus en plus differencié des structures agraires traditionnelles de la "pampa" (FIGURE 4-p.38)

Buenos Aires constitue en cette année-là un espace assez compact avec une superficie de $24 \mathrm{~km} 2$. L'habitat contigu atteint Barracas vers le sud et Plaza de Miserere vers l'ouest. Les moyens de transport contribuent à relier de vieux axes de croissance: Flores depuis 1890 et Belgrano depuis 1900. En dehors de la Capitale Fédérale les sous-centres les plus importants dans la dernière décennie du XIXème siècle sont Quilmes, Lomas de Zamora et Morón. En 1895 le tissu urbain couvre $60 \mathrm{~km} 2$, chiffre qui dépasse $85 \mathrm{~km} 2$ si l'on ajoute la totalité des sous-centres périphériques connectés par le tramway et le chemin de fer. Les exploitations maraîchères continuent à être significatives et les fours à briques prennent aussi de l'importance Cette couronne, presque continue, se déplace au fur et à mesure du progrès des lotissements urbains. (FIGURE 4)

Les caractéristiques du milieu jouent un rôle central dans la différentiation spatiale. Les coteaux légers dits "lomas" sont choisis pour l'établissement des quartiers les plus aérés. Les trajets ferroviaires renforcent cette tendance. La petite vallée du Maldonado est un obstacle à l'expansion continue vers le nord. La zone la plus inondable avec le plus de risques sanitaires est choisie comme aire industrielle presque exclusive dans cette période. Il s'agit de la vallée du Riachuelo. (FIGURE 4 ) 
Quels sont les chiffres les plus répresentatifs du changement ? Sans doute s'agit-il du peuplement de la ville et de l'accroissement du nombre d'employés industriels. La population double en quinze ans, passant de 330.000 habitants en 1880 à 660.000 en 1895. (FIGURE 5 -p.39) Vers 1914 , il y a 1.500 .000 habitants dans la Capitale Fédérale, chiffre qui atteint les deux millions si on considère les municipes voisins. Le nombre d'employés industriels passe de 42.000 en 1887 à 130.000 au début du XXème siècle, pour atteindre environ 200.000 en 1914, dont les trois quarts se trouvent à la Capital Federal.

Quelles sont les déterminants principaux des mutations industrielles à Buenos Aires ? Il y a deux groupes de facteurs. L'un lié au modèle "agro-exportateur"; l'autre, à l'accroissement vertigineux du marché de consommation urbaine.

La décennie de 1880 représente la première période de radication des frigorifiques sur le front fluvial du Paraná Inférieur. Les grands établissements s'installent entre 1902 et 1905 et, aussi, durant la décennie de 1910, en profitant du réseau ferroviaire et des infrastructures portuaires: Avellaneda, La Plata et Zárate, représentent trois des quatre centres frigorifiques les plus importants d'Argentine.(Ortiz; 1978:378)

Parmi les six groupes d'industries, l'industrie frigorifique enregistre la majeure concentration, en ce qui concerne la taille de l'établissement; et aussi la supérieure croissance. Entre 1908 et 1935, la force motrice croît de $410 \%$, la valeur de la production, de $440 \%$, le capital investi, de $530 \%$ et le nombre d'ouvriers, de $1000 \%$, en passant de 5.700 à 56.700 pour toute l'Argentine. (Dorfman; 1982:296) C'est la période des fortes exportations de viande.

L'explosion démographique durant cette période comporte l'augmentation des volumes de la consommation totale. Entre 1895 et 1913 le marché intérieur croît plus rapidement que l'industrie nationale: les importations se multiplient par cinq pendant ces années.(Dorfman; 1982:291)

Les premiers grands établissements s'installent dans les mêmes quartiers du "saladero" et du "frigorifique": Barracas et Barracas au Sud. Entre 1880 et 1920 on compte dans la région 23 industries d'importance; dont, 15 se trouvent aux abords du Riachuelo. Signalons l'usine textile "Alpargatas" en 1885; les établissements alimentaires "Bagley" en 1892, "Molinos" en 1900, "Canale" en 1910 et "Terrabusi" en 1919; et les usines métallurgiques "Tamet" en 1902 et "Gurmendi" en 1919. (Schvarzer; 1980:225) (FIGURE 5-p.39) 
Dans presque tous les cas la radication des premières grandes industries signifie l'introduction de nouvelles technologies par rapport à celles des petits et moyens établissements existants.

Les mutations industrielles ne sont pas déterminantes des fortes transformations territoriales de Buenos Aires et de ses environs. L'influence directe concerne la définition d'une vocation urbaineindustrielle dans deux espaces régionaux: Barracas-Avellaneda et Ensenada-Berisso.

Dans les deux cas les ports et la connexion ferroviaire avec la pampa contribuent à définir la dite vocation. Un habitat précaire très particulier se développe aux alentours: les logements en tôle de zinc avec un haut niveau d'entassement et très peu de services. La pollution et la dégradation générale du milieu acquièrent une grande ampleur. (FIGURE 5-p. 39 )

En ce qui concerne la production primaire intensive, elle a un grand développement dû principalement à deux raisons: l'accroissement du marché de consommation urbaine et la lente incorporation des agriculteurs étrangers, surtout italiens du sud, à la production maraîchère. Il n'y a pas d'interventions publiques planifiées, sauf l'"ejido de chacras y quintas" de La Plata en 1882. La subdivision des grandes exploitations d'élevage les plus proches de la ville ne se fait pas seulement en lotissements urbains, mais aussi en petites parcelles, généralement entre 5 et 20 ha. Dans la plupart des cas, ces espaces productifs allaient être très vite déplacés à cause de l'expansion urbaine: ce qui ne constitue pas une reconversion productive. Par contre la tendance est à la continuité avec les générations successives d'agriculteurs de modes de production traditionnels durant plus d'un demi-siècle. La "ceinture maraîchère", même discontinue subit des déplacements lents mais continus entre 1890 et 1960 . La relation "transformation territoriale-mutation productive" est subordonnée totalement au déploiement des autres processus dérivés de l'expansion urbaine; principalement la consolidation de Buenos Aires comme centre politique, administratif, commercial, portuaire et industriel.

4 La ville cosmopolite

et la première substitution d'importations 
Depuis la décennie de 1920, Buenos Aires est la première ville latinoaméricaine en ce qui concerne la radication de grandes enterprises étrangères, et leur importance comme centre culturel et commercial.

La concentration de richesses, produit du modèle "agro-exportateur" à Buenos Aires, se vérifie dans la qualité et la couverture de services urbains. Au capital investi en infrastructures et équipements, s'ajoute la capacité d'épargne d'un nombre significatif d'immigrants européens.

Comment pourrait-on expliquer l'installation de "Ford", de "General Motors", de "General Electric" et de "Colgate Palmolive" vers 1920 dans un pays totalement périphérique au marché de ce moment-là, si ce n'est de cette façon?

Cette mutation industrielle continue de manière graduelle, mais permanente, durant les deux décennies suivantes. Il faut souligner la radication d'autres grandes entreprises dans des quartiers et localités plus éloignées des aires urbaines consolidées, même des aires industrielles traditionnelles: "Firestone" (1931) et "Ducilo" (1935) et "Astilleros Rio Santiago" (1936) au sud de la région; "Grafa" (1931), "Atanor" (1939) et "Philips" (1945) au nord; et "Good Year" (1930) et "Chrysler" (1946) à l'ouest.(Schvarzer; 1980:227) Par contre, les nombreux ateliers et petites entreprises prolifèrent particulièrement dans la ville de Buenos Aires proprement dite. (FIGURE 6-p.40)

En 1941 se maintient encore dans la région la proportion d'employés industriels de 1913: les trois quarts des 410.000 ouvriers sont dans la Capitale Fédérale. Ici, l'expansion industrielle atteint son maximum en 1946: presque 400.000 employés. C'est le temps de la démarche industrielle vers la périphérie à la recherche de parcelles grandes et moyennes.

En ce qui concerne les transformations territoriales, elles sont très importantes: Buenos Aires enregistre un accroissement démographique explosif, mais ce qui est encore plus étonnant c'est l'expansion territoriale dûe au processus de subdivision de la terre. (FIGURE 6)

Buenos Aires est, en 1934, la septième ville du monde avec 3 millions d'habitants. La superficie urbanisée dans la région passe de $85 \mathrm{~km} 2$ vers 1895 à $680 \mathrm{~km} 2$ en 1940. Tandis la population et l'expansion urbaine se multiplient respectivement par cinq et par huit. (FIGURE 6)

Ce processus de réduction de la densification et les caractéristiques du système de transport public, seront significatifs dans la définition d'un 

productive".

Cette période marque la consolidation industrielle de BarracasAvellaneda et d'Ensenada-Berisso, les deux aires les plus proches des principaux ports de la région. Mais, elle représente aussi la formation d'un tissu mixte, où le résidentiel côtoie avec des ateliers et de petites industries dans une proportion importante dans la Capitale Fédérale. L'installation des grandes entreprises périphériques représente le début d'une tendance qui sera consolidée pendant les années cinquante.

En 1935 la Capitale Fédérale apportait $47 \%$ du total de l'industrie manufacturière nationale, contre $46 \%$ en 1946 et $39 \%$ en 1954 . En ce qui concerne les Partidos du Gran Buenos Aires, ils contribuaient avec 17\%, $24 \%$ et $27 \%$ pour les années mentionnées. Vers 1935, la moitié de l'industrie "porteña" était distribuée dans cinq des ses vingt quartiers. Tandis que $60 \%$ de l'industrie périphérique se situait à AvellanedaLanús. La population passe de 19.000 habitants en 1895 à 145.000 en 1914 et 500.000 en 1947.

Durant les décennies de 1930 et 1940, la "spécialisation territoriale" est assez forte: la localisation prochaine "industrie-résidence ouvrière" est révélatrice, d'une part, d'un réseau de transport public déficient, et d'autre part, de la disponibilité de terres vacantes près des établissements.(FIGURE 6)

En ce qui concerne la production agricole intensive, de nombreuses petites exploitations alternent avec des localités et des stations ferroviaires. Vers 1937, les "Partidos" avec les plus nombreuses exploitations intensives de moins de 25 ha, les dites "huertas", "quintas" et "chacras" configurent une ceinture discontinue, où se détachent Merlo, General Sarmiento et La Matanza avec 239, 169 et 146 exploitations, soit $63 \%$, $44 \%$ et $51 \%$ de leur total respectif. (FIGURES 10b et 12b) L'information des recensements de 1937 et 1947 (R.ArgentinaCNA;1937) Il faut citer également Luján, Exaltación de la Cruz, Cañuelas et La Plata plus éloignées avec respectivement 287, 238, 137 et 133 exploitations. (FIGURE 10b)

La caractéristique de l'activité durant la période de l'entre-deux guerres est la constitution de groupes d'agriculteurs étrangers, particulièrement des Italiens et des Espagnols qui accèdent à la propriété avec des exploitations de petites dimensions. L'arrivée des premières familles d'agriculteurs du nord argentin se produit vers la fin de la décennie de 1940 elles s'incorporent en tant que travailleurs 
temporaires. Les autres activités -floriculture, aviculture- ne se développent pas encore pendant cette période.

Quelles sont les mutations les plus significatives en ce qui concerne la réconversion des processus productifs entre 1920 et la fin des années 1940 ?

La production agricole continue avec les mêmes techniques traditionnelles, fondée sur la main d'oeuvre intensive et la faible incorporation de technologies.

Les changements de l'industrie supposent l'introduction de technologies deja répandues en Europe et en Amérique du Nord. Pour Buenos Aires, cette mutation représente un fort impact économique et social: il s'agit de la prolifération de grands établissements avec des processus productifs "tayloriens" où le trait dominant est l'incorporation de nombreux ouvriers peu qualifiés.

\section{La démarche vers la périphérie et la deuxième substitution d'importations}

Entre 1945 et 1975 Buenos Aires est la principale ville d'attraction du Cone-Sud Américain. Le processus migratoire européen est remplacé par un autre, provenant de l'intérieur argentin et des pays limitrophes: Paraguay, Bolivie, Uruguay et Chili. Le Grand Buenos Aires passe de 4,7 millions d'habitants en 1947 à 6,8 en 1960 et 8,5 en 1970 .

L'industrialisation sustitutive s'accentue durant de brèves périodes, particulièrement en 1943-1950 et 1957-1964. C'est le temps du transport public collectif et de la prolifération de lotissements sans services. Entre 1940 et 1962 la région métropolitaine passe de 680 à $1450 \mathrm{~km} 2$. Cette situation signifie que la superficie lotissée des Partidos du Grand Buenos Aires se multiplie presque trois. De l'expansion territoriale résulte un espace caractérisé par de nombreux quartiers éloignés et mal reliés au centre-ville. (FIGURE 7)

Dans quelle mesure les mutations productives produisent des transformations territoriales ? Il s'agit de la période connaissant les changements les plus forts à cause de l'expansion industrielle et agricole intensive.

Parmi les grands établissements, se détachent "BGH" (1954), "Fate" (1957), "Ford" (1959) et "Dálmine Siderca" (1960) au nord; "Mercedes Benz" (1952), "Schcolnik" (1952) et "Borgward" (1954) à l'ouest; 
"Sasetru" (1950), "Alpargatas" (1953), "Peugeot" (1963) et "Petroquímica Grl.Mosconi" (1974) au sud. (Schvarzer; 1978:227).

Le processus d'industrialisation est aussi important en ce qui concerne la prolifération de petits et moyens établissements ce qui se traduit par la création de la "première couronne industrielle", ainsi que six "axes industriels" Vers 1954 la configuration était celle d'une ceinture tout le long des Partidos limitrophes à la Capitale Fédérale et des lignes radiales qui suivent six voies d'accès à Buenos Aires (Chiozza;1977:257). (FIGURE 7-P.41) En ce qui concerne la Capitale Fédérale, un développement industriel se produit vers les quartiers ouest situation qui signifie la diminution du pouvoir traditionnel des quartiers sud.

La radication industrielle dans la Capitale Fédérale commence à être limitée par le "Code de Bâtiment" de 1944, et par des lois sucessives d'interdiction d'installation d'industries à partir de 1972 (Loi 20.560 et décrets postérieurs). Ce qui ne signifie pas pour autant une démarche vers l'intérieur de l'Argentine. Durant cette periode l'industrialisation suburbaine est très forte: 215.000 employés en 1946, 293.000 en 1954 et 500.000 en 1974; ce chiffre atteint 625.000 employés si on considère les 34 Partidos de la région.

Les établissements étaient encore principalement des industries de main d'oeuvre intensive. La fin des années cinquante est celle de l'importation de biens de capital. La motorisation et la mécanisation industrielles s'accélerent après la Seconde Guerre Mondiale; particulièrement en ce qui concerne des branches assez nouvelles: métaux, automotrice et machines. Cette situation ne change pas la tendance générale de forte occupation industrielle; il existe également un ralentissement du rythme des emplois secondaires.

Dans ce cadre, le rapport "transformations territoriales-mutations productives" se consolide avec la diffusion du transport public collectif dans le Grand Buenos Aires. Il s'agit de la période où la résidence ouvrière s'éloigne de l'industrie: les voyages foyer-travail ont, très souvent, des durées de 1 à 2 heures. Vers la fin de la période, Buenos Aires est la métropole qui posséde la plus grande quantité de lots inoccupés du monde: entre 4 et 5 millions.

Le début de la décennie 1970 est celle du plus fort développement industriel. La Capitale Fédérale, avec les établissements à plus haute valeur ajoutée et les moins polluantes. Les Partidos de la "première couronne" avec des établissements de tailles variées et certaines spécialisations: textile á San Martín et Vicente López, materiel de transport à Tres de Febrero, Lanús et La Matanza, alimentaire et dérivés 
du pétrole à Avellaneda. Les Partidos les plus periphériques avec de grands établissements de production diverse: automotrice, petrochimique,chantiers navals, métallurgique, verrerie et alimentaire.

La décennie de 1960 est le début d'une tendance de grand développement des activités agricoles intensives, au diapason avec l'augmentation de la taille du marché intérieur. Il s'agit de la période d'expansion la plus importante de la production horticole et de l'introduction de nouvelles techniques de production intensives pour la région métropolitaine: la floriculture depuis 1958-1960 et l'aviculture commerciale depuis 1966-1968. "En 1960, 71\% des terres rurales de 1947 étaient déjà urbanisées; il restait moins de $15 \%$ de la superficie de ces Partidos utilisée pour des exploitations agraires"(Gutman et al;1987:46)

L'horticulture est encore l'activité la plus répandue: entre 18.000 et 20.000 ha vers 1969. Le sud-est produit plus de 50\% de la région: La Plata, F.Varela et Berazategui. D'autres zones importantes se trouvent vers le nord-ouest: Escobar, Pilar, Marcos Paz, General Rodríguez et Luján. (FIGURES 11a, 13a, 14b et 15b) La consolidation de grands producteurs, généralement italiens, représente le début d'une différentiation à l'intérieur du circuit économique, en ce qui concerne le contrôle de la commercialisation. La diffusion de produits agrochimiques et de pesticides est postérieur.

L'essai avorté de concrètion du Marché Central de Buenos Aires depuis 1967 et l'ouverture du Marché Régional de La Plata vers la fin de cette période sont deux cas qui témoignent de la volonté d'amélioration de la gestion commerciale des fruits et des légumes pour un marché de dix millions d'habitants. Un des problèmes principaux à résoudre est celui du secteur des transporteurs intermédiaires, les "consignatarios"

La diffusion de la floriculture à partir de la moitié des années cinquante est une réponse à la diffusion généralisée de certains modes de consommation dans la société argentine; mais aussi à l'impulsion données par des agriculteurs portugais et, un peu plus tard, japonais. L'introduction de couvertures plastiques vers les années soixante-dix contribue à changer le paysage péri-urbain en peu temps. Les zones les plus importantes sont: Escobar-Pilar et La Plata-F.Varela.

Le début de l'aviculture commerciale à moyenne et grande échelles date des années soixante. Elle concerne les aires les plus periphériques de la région metropolitaine: particulièrement Exaltación de la Cruz et Cañuelas. Les établissements moyens se développent de manière explosive vers les années soixante-dix. La spécialisation et la 
diversification croissante en matière de produits de ferme est postérieure à cette periode.

Quelle rapport "transformation

territoriale-mutation productive" peut-on définir à partir de ces changements ? Il existe un processus de subdivision de la terre très important, et peu connu, en petites exploitations rurales. Il s'agit de nombreuses parcelles fractionnées soit pour une future spéculation foncière urbaine, soit pour l'usage productif, soit pour le loisir et la récreation. La tendance observée semble indiquer un décalage assez fort entre la "production" de terres rurales périurbaines et leur utilisation à des fins productives.

Le développement de productions intensives pendant cette période déterminent les transformations territoriales périurbaines. Mais le résultat -la subdivision sans discrimination- dépasse les expectatives et les demandes de terre pour des producteurs et pour des propriétaires de résidences secondaires. La spéculation foncière est donc le principal déterminant des transformations territoriales durant cette période.

\section{LA TERTIARISATION METROPOLITAINE, LA DESINDUSTRIALISATION ET LA RECONVERSION PRODUCTIVE}

Depuis 1975 la région métropolitaine traverse une période de crise qui atteint la majeure partie de la population. La dette externe, la "financiérisation" de l'économie et la difficulté d'accès à des marchés internationaux de plus en plus protectionnistes, contribuent à une réduction de taille du marché de production et de la consommation locale.

A Buenos Aires, les manifestations les plus remarquables de ce processus sont la réduction des niveaux d'investissements en infrastructures et équipements, la désindustrialisation, la tertiarisation des petits commerces, une administration publique hypertrophiée, et la prolifération d'emplois "informels"

La nouvelle politique d'ajustement, particulièrement la privatisation des entreprises publiques et les termes de la flexibilisation de l'emploi ont contribué à accentuer les différences sociales. Actuellement il existe des indices d'une reconversion économique assez forte. L'introduction de nouvelles technologies productives dans certaines branches produit un raccourcissement des distances qui séparaient les "mutations innovatrices" des pays centraux, des "mutations résiduelles" des pays périphériques. 
Quelle est le rapport "transformation territoriale-mutation productive" depuis 1975 ? La subdivision de la terre est presque arrêtée, le rythme de croissance démographique de la région est faible et la crise de l'industrie et des productions intensives s'accentue. Buenos Aires n'est plus le centre d'attraction des années cinquante et soixante. Le scénario est celui d'une ample aire urbaine de $145 \mathrm{~km}$ de longueur entre La Plata et Zárate où se trouvent de nombreuses friches industrielles et agricoles.

Au rétrécissement du marché foncier s'ajoutent en 1978 les effets de la Loi Provinciale 8912 d'Usage du Sol. La Région Métropolitaine de Buenos Aires enregistre dans les années quatre-vingts, plus de dix millions de lots urbains (McAuslan; 1987) et le tiers de toutes les exploitations de moins de 25 ha de la Province. Il faut souligner que cette proportion serait plus forte si on pouvait y inclure la totalité des proprietés rurales, compte tenu du nombre important de petites parcelles non utilisées.

La population de toute la région passe de 9,2 millions en 1970 , à 10,7 en 1980 et 12,3 en 1991. Les Partidos de plus grande croissance sont les plus périphériques: en vingt ans Pilar et Escobar multiplient par quatre leur nombre d'habitants, Moreno, Florencio Varela et Marcos Paz presque par trois. Par contre, la Capitale Fédérale et les Partidos de la première couronne enregistrent un taux presque nul: de 4,7 millions à 4,8 entre 1970 et 1991 .

La superficie des 35 municipes qui appartiennent à la région métropolitaine est de $12961 \mathrm{~km} 2 ; 2168 \mathrm{~km} 2$ sont des espaces urbains, $2238 \mathrm{~km} 2$ sont périurbains. Une analyse intra-régionale montre la différentiation selon les couronnes et l'agglomération de La Plata. Tandis que les 19 Partidos traditionnels du Grand Buenos Aires ont $1537 \mathrm{~km} 2$ urbains et $956 \mathrm{~km} 2$ periurbains, les Partidos de la troisième couronne enregistrent $363 \mathrm{~km} 2$ urbains et $988 \mathrm{~km} 2$ périurbains. Le Grand La Plata a $268 \mathrm{~km} 2$ urbains et $294 \mathrm{~km} 2$ périurbains. (FIGURE 1-page 8 )

La grande extension de la région métropolitaine croît plus encore si on considère l'augmentation relative $d u$ coût $d u$ transport public et l'éloignement des lieux de travail. Il y a une tendance au rétrécissement du marché de l'emploi dans l'espace suburbain et une offre de travail informel dans la ville de Buenos Aires.

La désindustrialisation est presque commune à toute la région. Les établissements se réduisent de 56992 à 47640 entre 1974 et 1985 . Le nombre d'employés passe de 964.200 à 764.600 pour la même période. (FIGURES 8 et 9-pp.42-43) 
La tendance à la spécialisation dans la fonction résidentiel et le secteur tertiaire de la Capitale continue: des 40.000 établissements industriels des années soixante, en subsistent 16100 en 1985, tandis que la ville perd 100.000 emplois secondaires en 10 ans.

La zone industrielle traditionnelle d'Avellaneda-Lanús perd 21.000 postes; le nord de la première couronne, en perd 30.500 et l'ouest, 10.500. Par contre, certains Partidos de la deuxième et la troisième couronnes enregistrent des tendances d'industrialisation significatives, particulièrement vers le nord. Il s'agit de Campana, Pilar et Escobar avec 7100 nouveaux emplois. Les Partidos du sud de la région perdent 20.200 postes. (FIGURES 8 et 9)

La récente intégration de l'Argentine au Mercosur signifie aussi les changements de certains termes de production à cause de l'agrandissement du marché et des différents niveaux de concurrence selon les branches industrielles dans chaque pays. C'est le début des stratégies d'entreprises régionales, particulièrement en Argentine et au Brésil.

Les trois dernières années constituent le début d'une tendance de mutation industrielle partielle et résiduelle. Partielle, parce qu'elle comporte un petit nombre de branches: l'informatique, les télécommunications, la pétrochimie et l'industrie automobile, en sont les plus remarquables. Ces dernières sont inclues dans les études de cas de cette recherche.

Mutation résiduelle parce qu'elle représente l'incorporation de technologies de production déjà introduites dans les pays les plus développés; même s'il y a eu une réduction du temps entre l'innovation et la diffusion. En tout cas, c'est la manifestation d'une tendance d'accélération globale des mutations productives. La "transnationalisation" et la "déterritorialisation" du capital, ne peuvent être observés en termes équivalents en ce qui concerne les nouvelles technologies.

Les grandes entreprises: introduisent-elles les mêmes technologies dans leurs filiales des pays développés et des pays en voie de développement ? L'analyse des cas peut conduire à répondre à certaines questions.

Les activités agricoles intensives traditionnelles connaissent une forte réduction des niveaux de production sauf l'aviculture qui 
reste presque stable. L'horticulture subit une réduction de l'aire cultivée: 15.000 ha vers le début des années quatre-vingts et 12.000 ha actuellement. La floriculture traverse une crise depuis 1972-1975. (FIGURES 11b, 13b et 16)

Depuis 1975 il y a une diffusion des sémences hybrides, pour la plupart importées, et une utilisation croissante des herbicides, des insecticides et des fertilisants. (Gutman; 1987:80). L'introduction de la production sous couverture plastique est assez récente pour l'horticulture; elle permet un meilleur contrôle climatique et sanitaire, au détriment d'une incorporation importante de capital. Maintenant l'exploitation à ciel ouvert est entre six à dix fois moins chère que la production sous couverture. Les différences de rendements concernent non seulement la productivité, mais aussi la qualité et la production toutes saisons.

La fin des années quatre-vingts sont le début d'une mutation productive ponctuelle mais certaine. Il s'agit d'une mutation dejà faite dans d'autres pays. Les dernières quatre années marquent l'introduction de productions intensives non-traditionnelles de la pampa: élevage de lapins, de loutres, de grenouilles et de certaines espèces fruti-horticoles nouvelles pour l'Argentine. A cela s'ajoute une diffusion plus large de l'apiculture, mais dans toute la pampa, à partir les années soixante-dix.

Malgré toutes ces mutations dans les productions intensives traditionnelles et non-traditionnelles, le principal déterminant des activités concerne le mode de travail de la terre et la commercialisation. La grande diffusion des contrats pour partager la production entre propriétaire et travailleur, connue comme "mediería", suppose une altération des relations de travail: la différentiation entre grands et petits producteurs, et en conséquence, des conditions d'exploitations de la force de travail, des familles d'agriculteurs du nord argentin, du Paraguay et de la Bolivie.

L'essai d'amélioration du système de commercialisation, materialisé en 1984 par l'ouverture du Marché Central de Buenos Aires à La Matanza, ne résoud pas la question. Le changement des canaux de commercialisation concerne théoriquement la substitution du "fréteur commerçant" par le "mandataire d'achats" (Iglesias; 1986:109). Mais, la figure antérieure persiste avec la prolifération des marchés clandestins dans toute la Région.

Friches industrielles et reconversion technologique partielle, d'une part; ralentissement de la production agricole intensive et 
début dans l'introduction de produits non-traditionnels, d'autre part; ces deux groupes de tendances démontrent l'action de la réduction du marché de consommation locale comme facteur des transformations territoriales. Les mutations productives par contre, sont assez récentes et peu généralisées pour définir des impacts territoriaux à échelle métropolitaine.

Les transformations territoriales conséquence des mutations productives sont ponctuelles. Les études de cas qui suivent sont les exceptions rares de ce rapport. Ici, la reconversion productive locale est très forte, il est donc pertinent d'étudier, malgré leur caractère nouveau, l'impact territorial par l'introduction de nouvelles technologies de production. 
FIGURE 2

SCHEMA IDEAL DE L'ORGANISATION

GENERALE DE L'ESPACE REALISE PAR GARAY

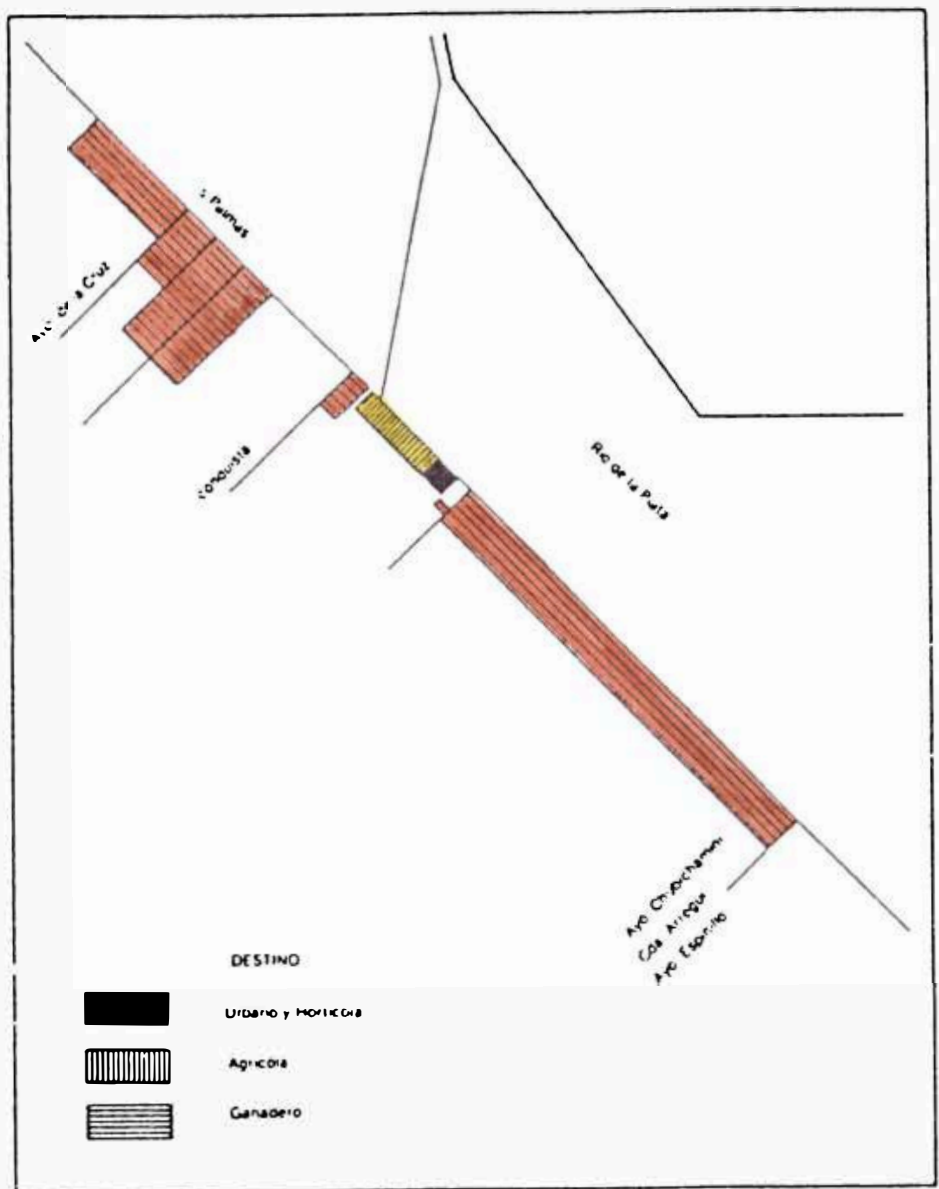

DESTINATION DES TERRES

URBAIN ET HORTICOLE

AGRICOLE

ELEVAGE

Source: Figueira, Ricardo (1983) "Del barro al ladrillo" In: Buenos Aires Historia de Cuatro Siglos. Ed. Abril, Buenos Aires. 
FIGURE 3

CARTE TOPOGRAPHIQUE DES ENVIRONS DE BUENOS AIRES

(PREMIERE MOITIE DU XIXème SIECLE)

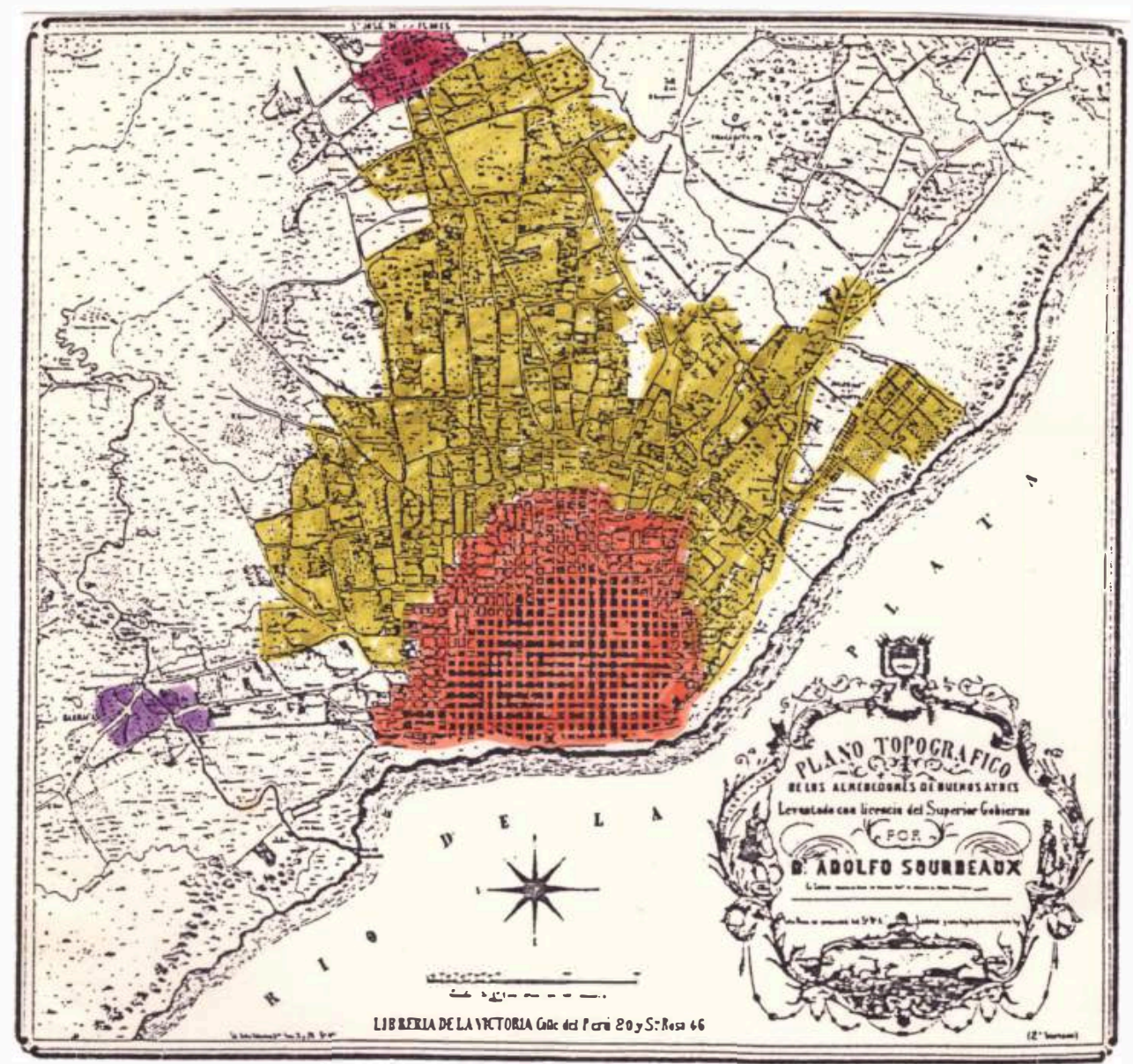

\section{BUENOS AIRES}

SAN JOSE DE FLORES

BARRACAS

AIRE MARAICHERE

Source: "Plano Topográfico de los Alrededores de Buenos Aires levantado con licencia del Superior Gobierno por A.Sourdeaux". In: Sarrailh, Eduardo (1983) "Lámparas y adoquines" Buenos Aires Historia de Cuatro Siglos. Ed. Abril, Buenos Aires. 
FIGURE 4

REGION METROPOLITAINE DE BUENOS AIRES

TRANSFORMATIONS TERRITORIALES

ET MUTATIONS PRODUCTIVES

$1870-1895$

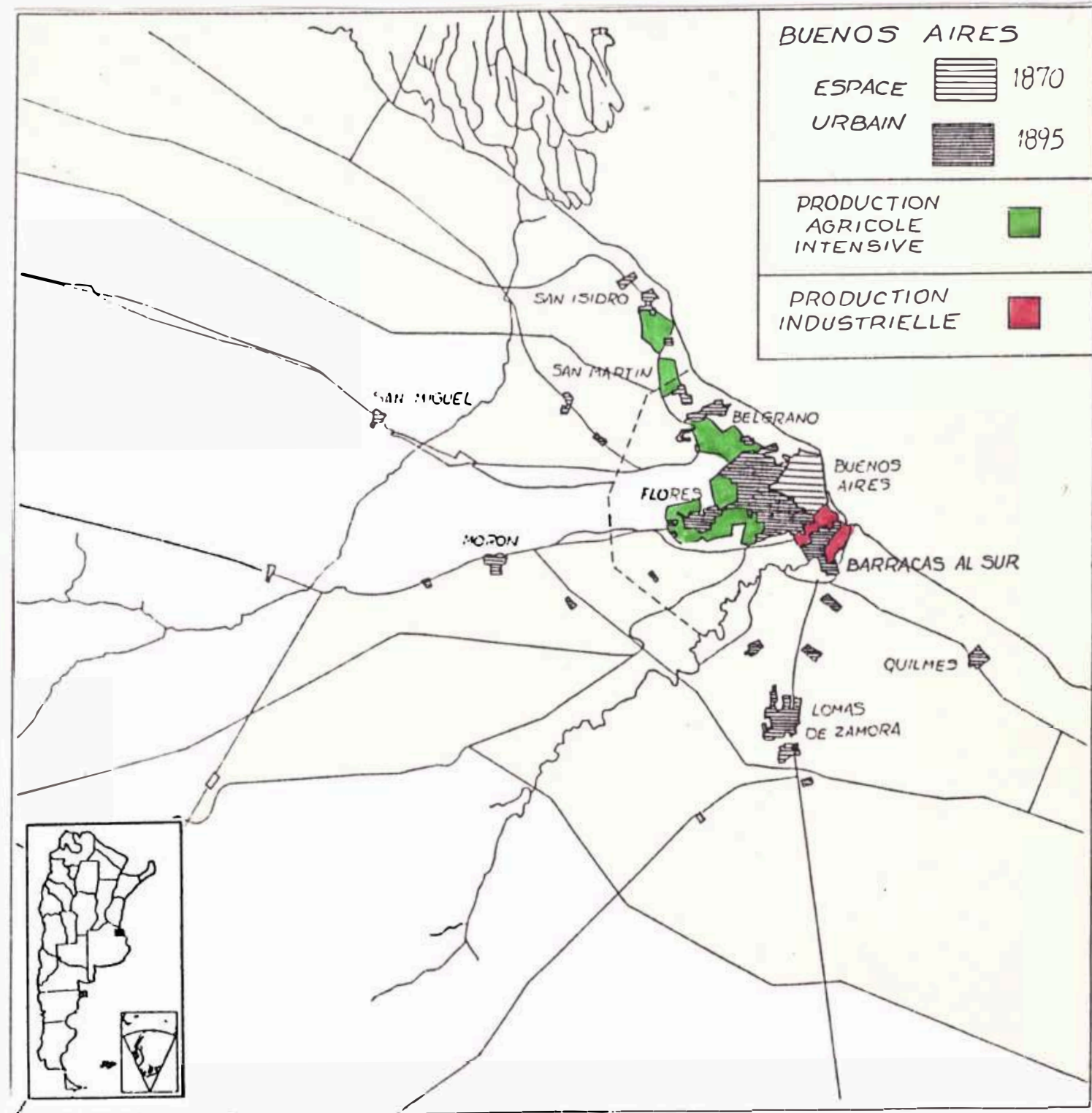

Sources: Espace urbain: Torres, Horacio (1978) Evolución de los procesos de estructuración espacial urbana. In: "Desarrollo Económico", Buenos Aires. Production industrielle et agricole: Elaboration personnelle à partir de bibliographies diverses. 
FIGURE 5

REGION METROPOLITAINE DE BUENOS AIRES

TRANSFORMATIONS TERRITORIALES

ET MUTATIONS PRODUCTIVES

\section{$1895-1915$}

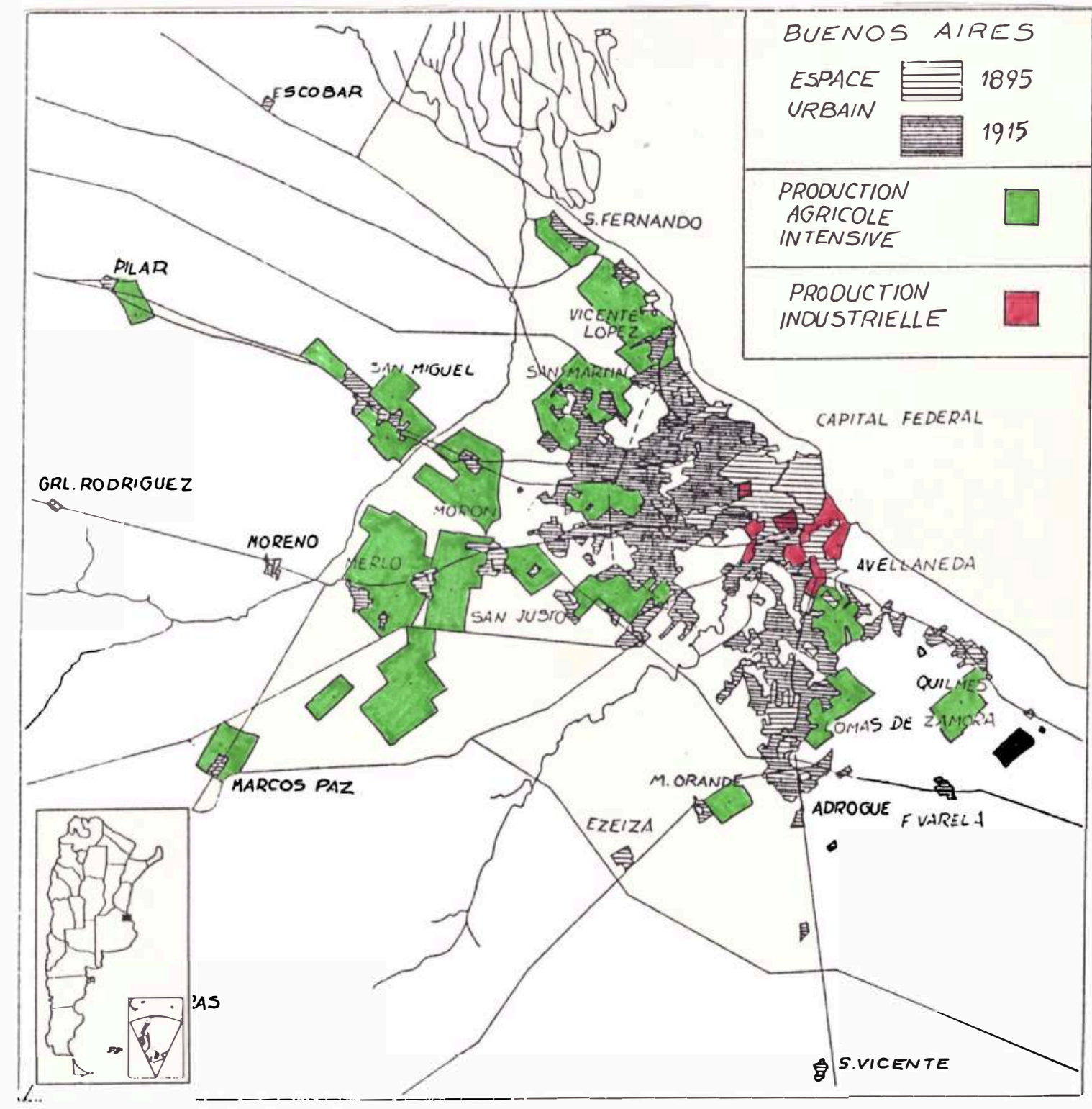

Sources: Espace urbain: Torres, Horacio (1978) op.cit. Production industrielle et agricele: Elaboration personnelle à partir de a) Schvarzer,J.(1983) La implantación industrial. In Buenos Aires Historia de Cuatro Siglos. b)Plano de los alrrededores de Buenos Aires de A.Vital et E.Roux. c) Randle, P. et N.Gurevitz (1979) Atlas Histórico de la Pampa Anterior. d) Censo Nacional Agropecuario-1914. e) Chiozza, E. El País de los Argentinos. Volume Région Métropolitaine. 
FIGURE 6

REGION METROPOLITAINE DE BUENOS AIRES

TRANSFORMATIONS TERRITORIALES

ET MUTATIONS PRODUCTIVES

\section{5 - 1939}

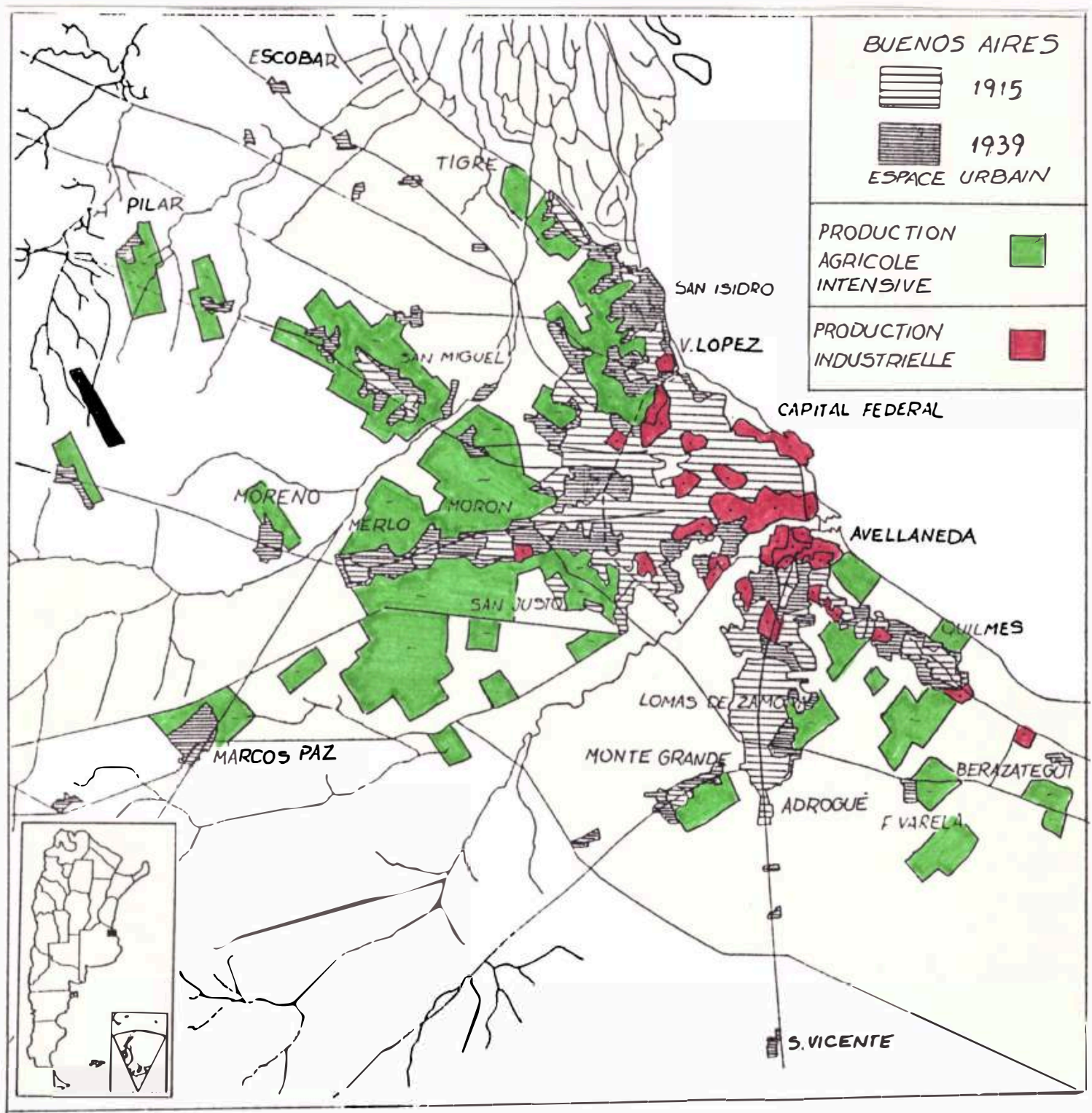

Sources: Espace urbain: Torres, H. (1978) op.cit. Productions industrielle et agricele: a) Schvarzer, J. (1983) op.cit. b) Censo Nacional Agropecuario 1914. c) Censo Nacional Agropecuario 1937. d) Chiozza, E. La integración del Gran Buenos Aires. In: Buenos Aires Historia de Cuatro Siglos. e) Chiozza, E. El País de los Argentinos. Volume Région Métropolitaine. f) Randle, P. et N.Gurevitz (1979) Atlas Histórico de la Pampa Anterior. 


\section{FIGURE 7}

REGION METROPOLITAINE DE BUENOS AIRES

TRANSFORMATIONS TERRITORIALES

\section{ET MUTATIONS PRODUCTIVES}

\section{$1947-1964$}

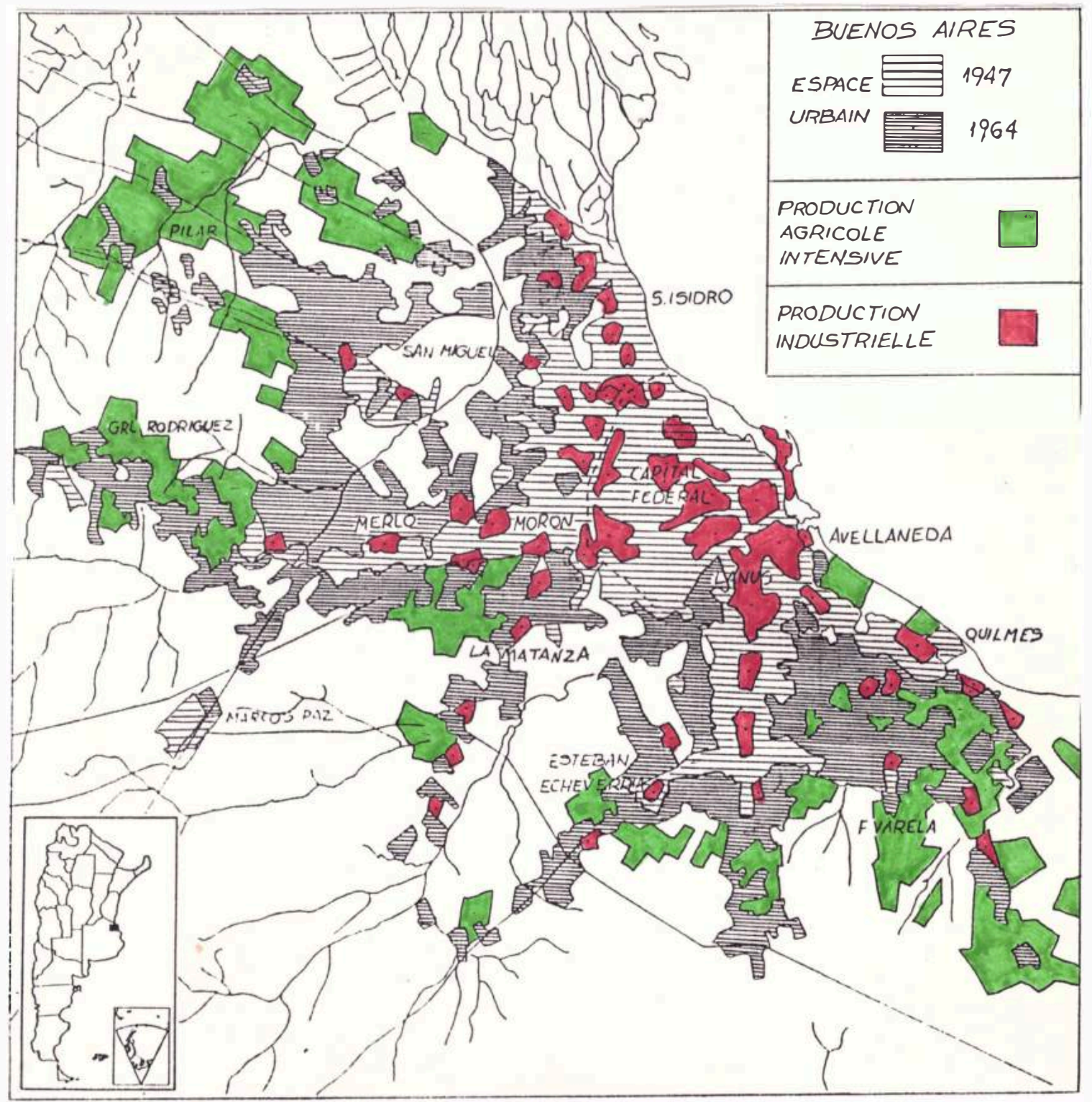

Sources: Espace urbain: Torres, H. (1978) op.cit. Productions industrielle et agricole: a) Carta Parcelaria Rural de la Provincia de Buenos Aires (1962) Dirección de Geodesia. b)Schvarzer, J. (1983) op.cit. c)Randle, P. et al (1979) op.cit. d) Chiozza, E. El País de los Argentinos...op.cit. 
FIGURE 8

REGION METROPOLITAINE DE BUENOS AIRES

EMPLOI INDUSTRIEL 1974

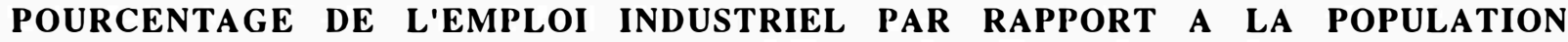
TOTALE

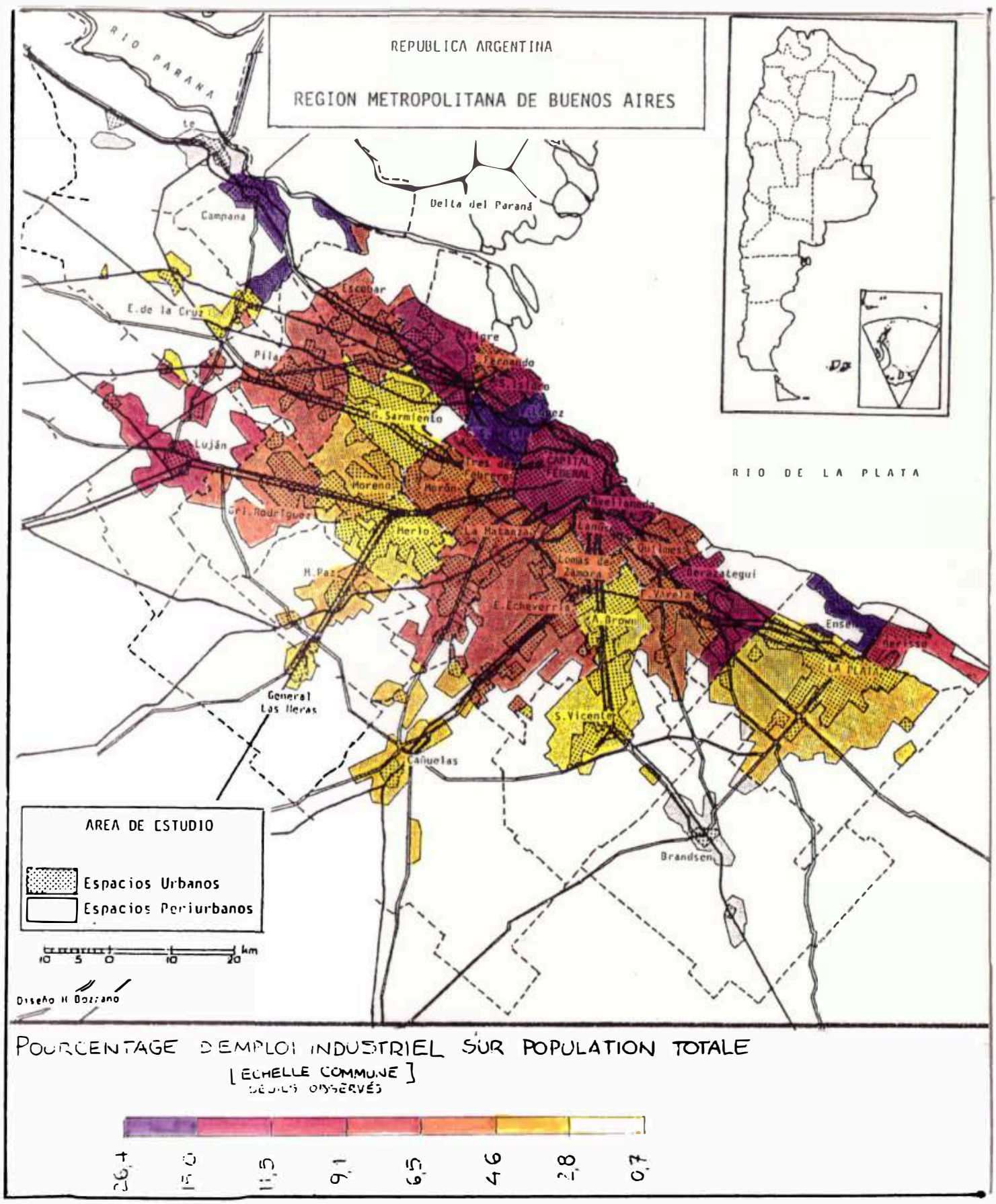

Source: Censo Nacional Económico 1974. Section Industrie. INDEC, Buenos Aires. 
FIGURE 9

REGION METROPOLITAINE DE BUENOS AIRES

EMPLOI INDUSTRIEL 1985

POURCENTAGE DE L'EMPloi INDUSTRIEl PAR RAPPORT A LA POPUlation TOTALE

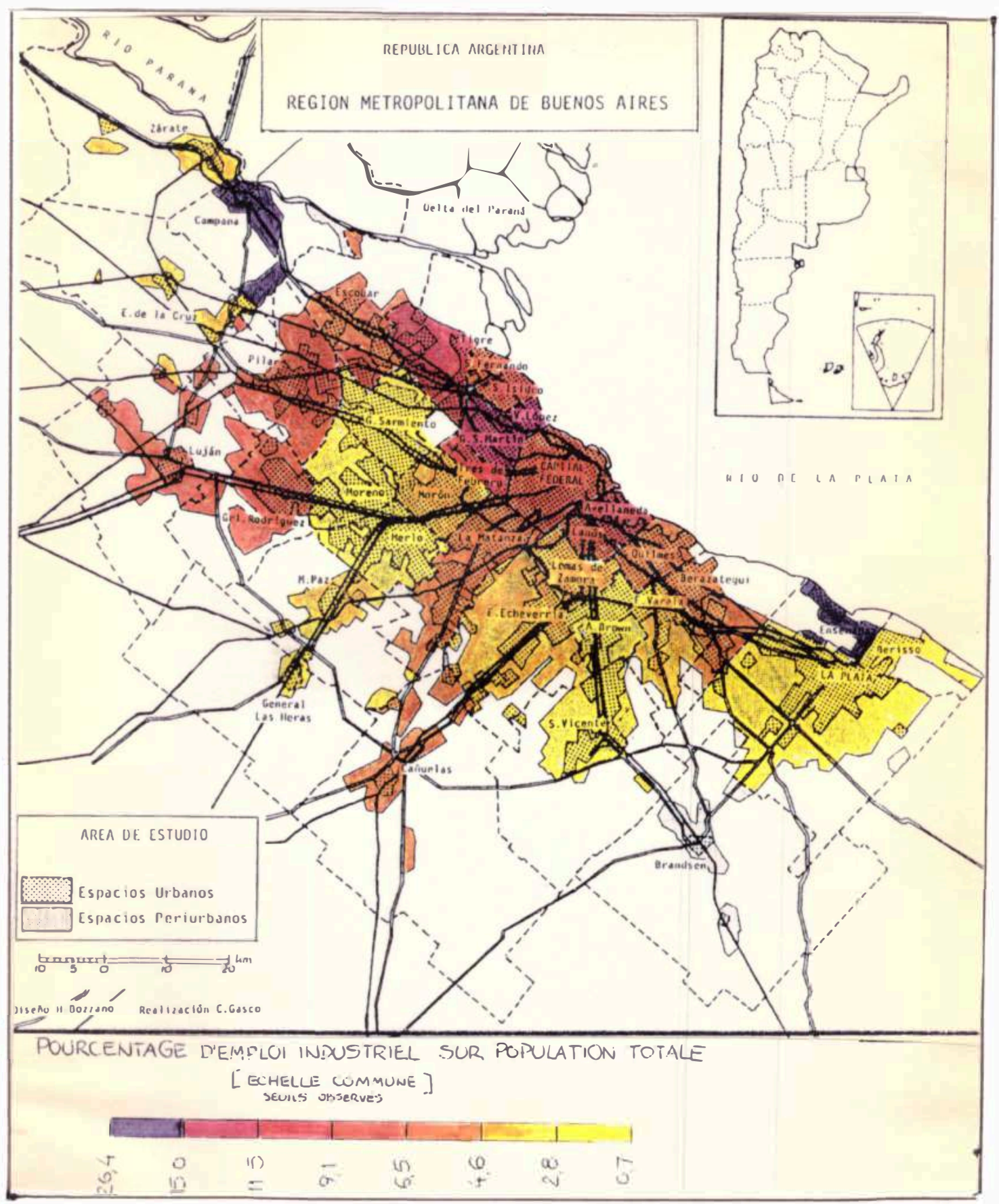

Source: Censo Nacional Económico-1985. Section Industrie. INDEC, Buenos Aires. 
FIGURE 10a

REGION METROPOLITAINE DE BUENOS AIRES

NOMBRE D'EXPLOITATIONS DE MOINS 25 HA (1914)
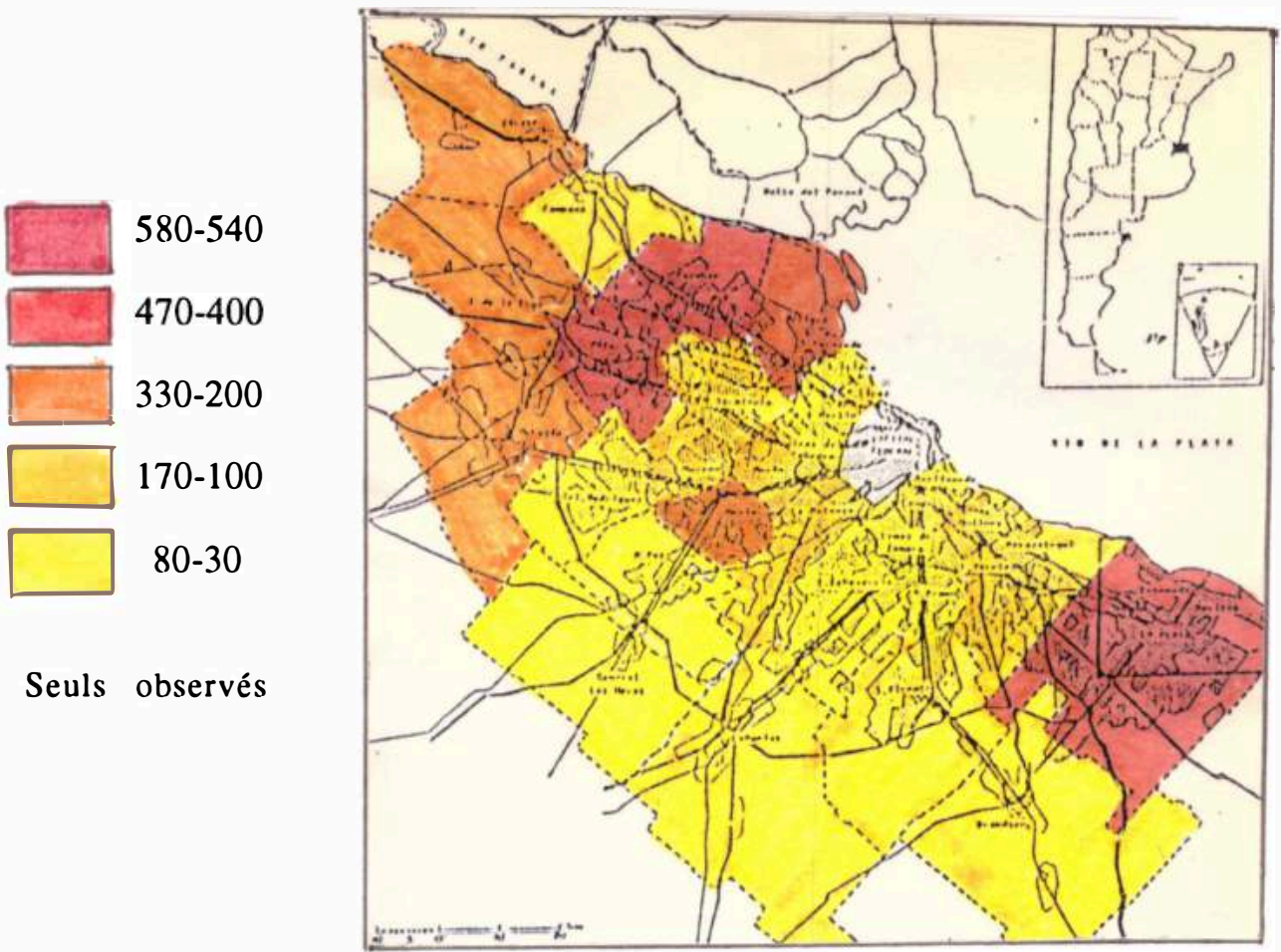

FIGURE 10b

REGION METROPOLITAINE DE BUENOS AIRES

NOMBRE D'EXPLOITATIONS DE MOINS 25 HA (1937)
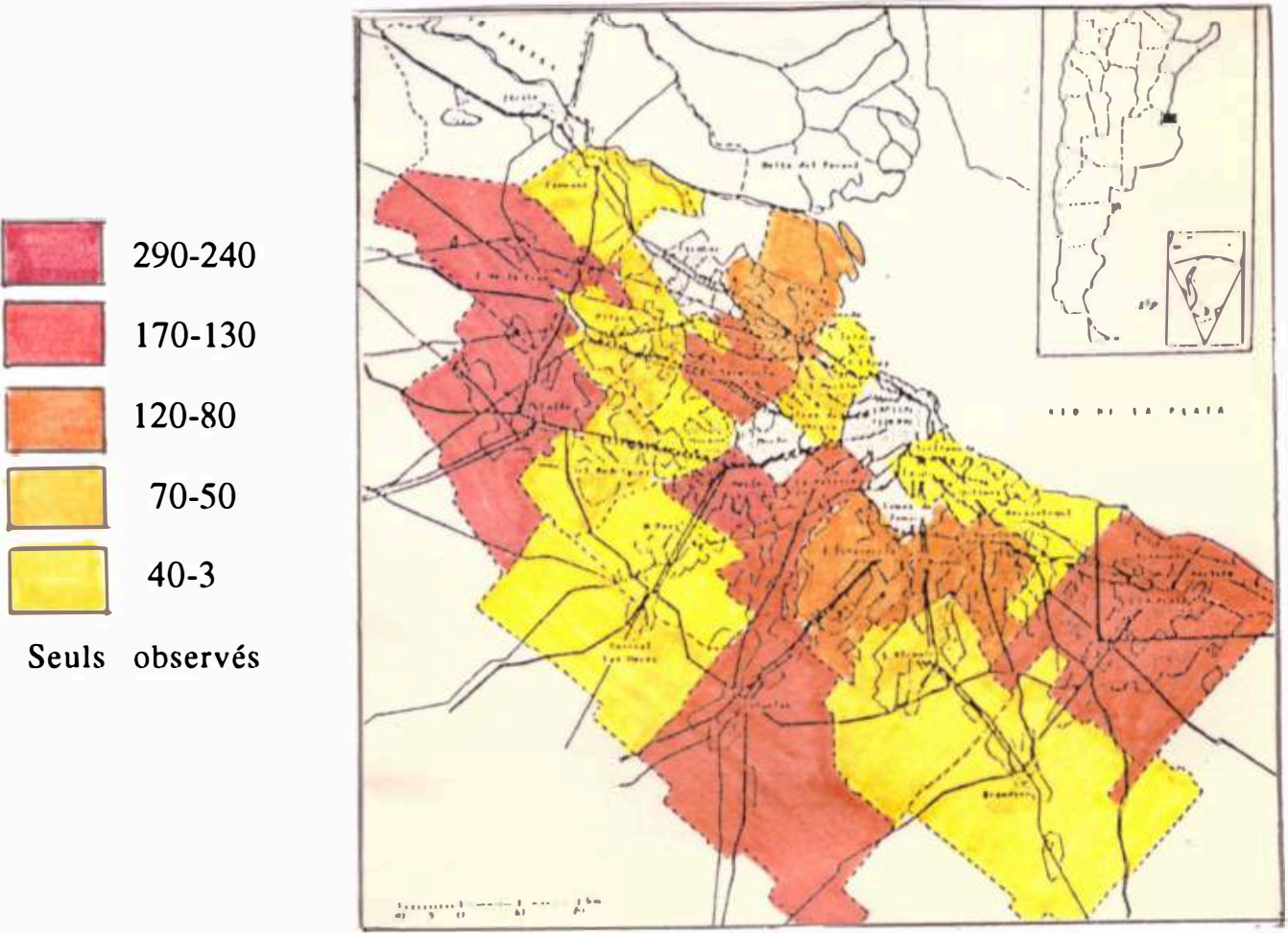

Sources:_Censos Nacionales Agropecuarios 1914 et 1937 
FIGURE 11a

REGION METROPOLITAINE DE BUENOS AIRES

NOMBRE D'EXPLOITATIONS DE MOINS 25 HA (1969)
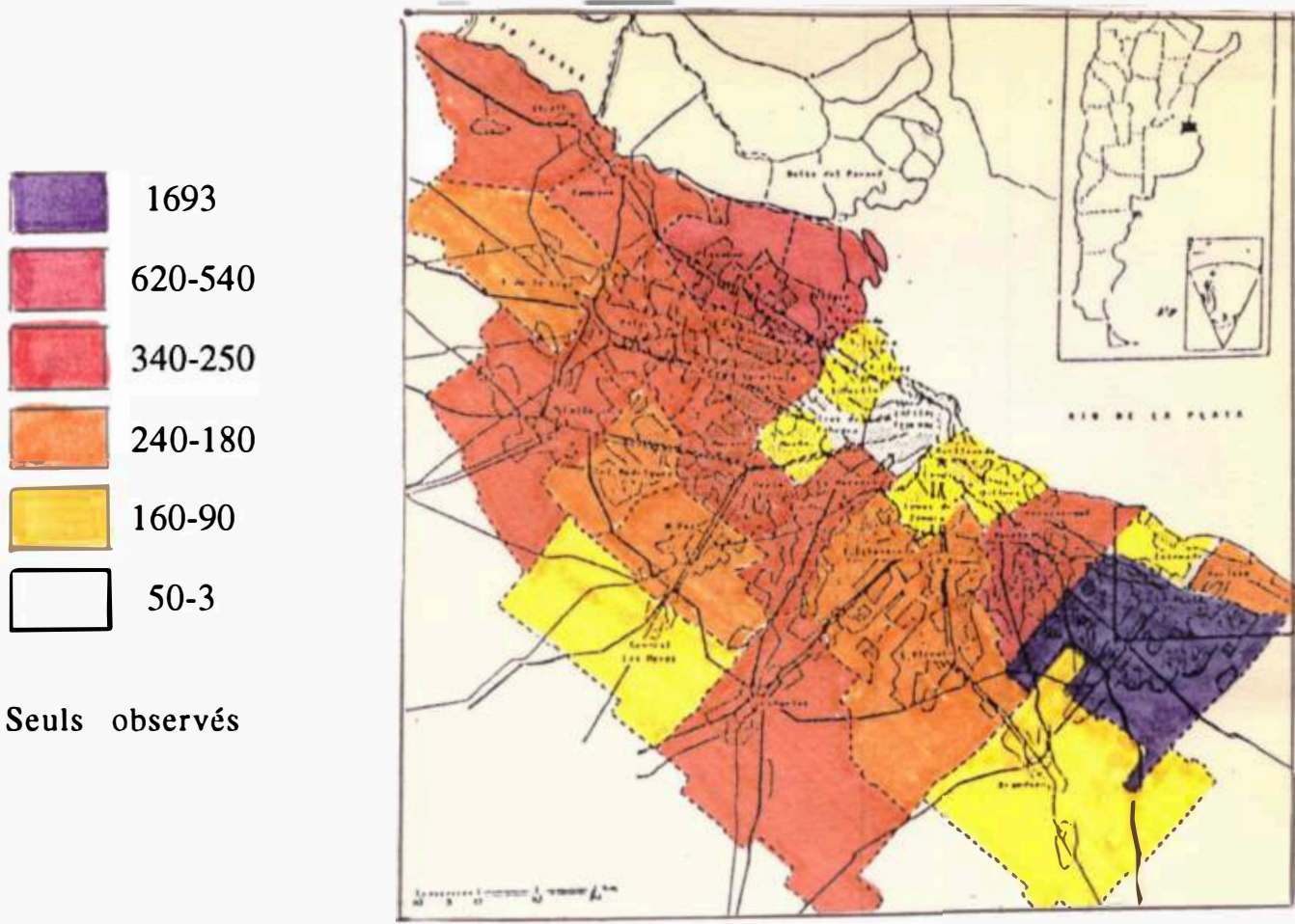

Seuls observés

FIGURE 11b

REGION METROPOLITAINE DE BUENOS AIRES

NOMBRE D'EXPLOITATIONS DE MOINS 25 HA (1988)

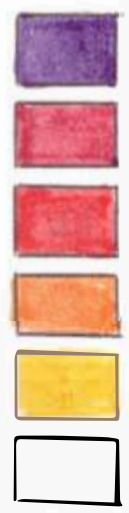

992

$530-370$

290

$180-140$

$120-60$

60-3

Seuls observés

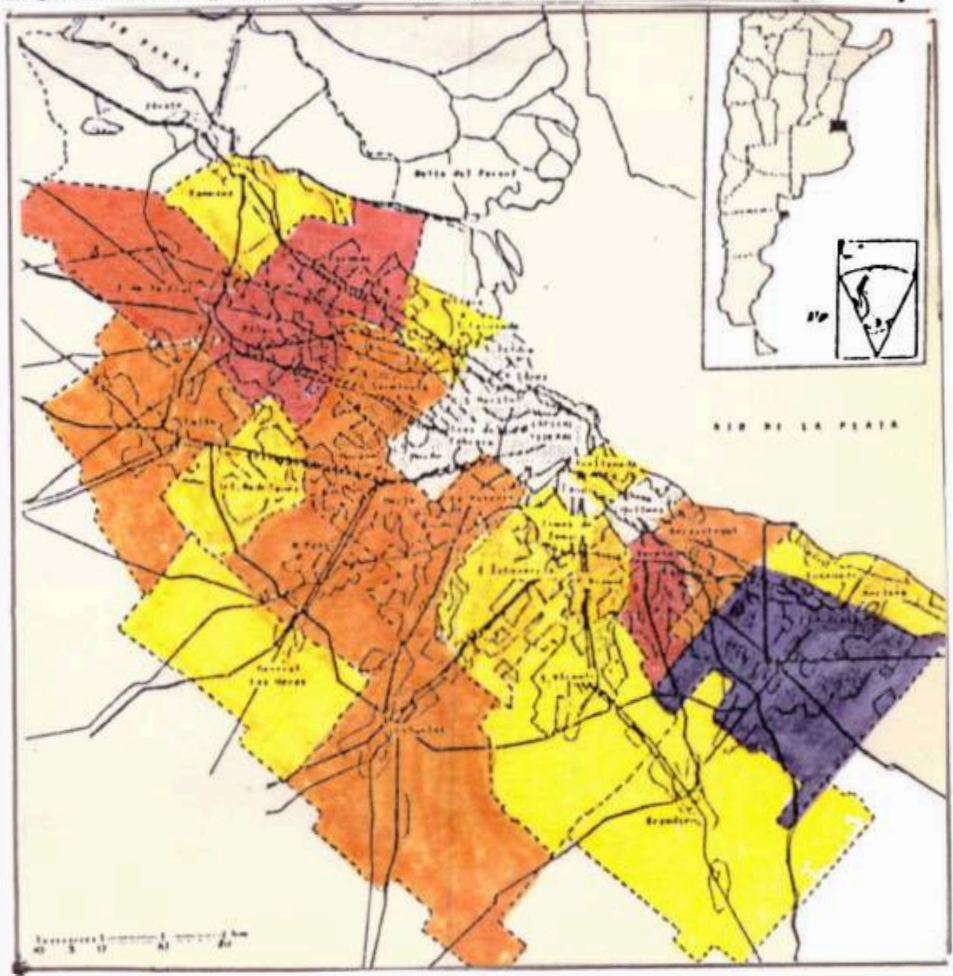

Sources: Censos Nacionales Agropecuarios 1969 et 1988 
FIGURE 12a

REGION METROPOLITAINE DE BUENOS AIRES

POURCENTAGE D'EXPLOITATIONS DE MOINS 25 HA

PAR RAPPORT AU TOTAL (1914)

$100-81 \%$

80-61\%

$60-41 \%$

$40-21 \%$

20-0\%

Echelle comune

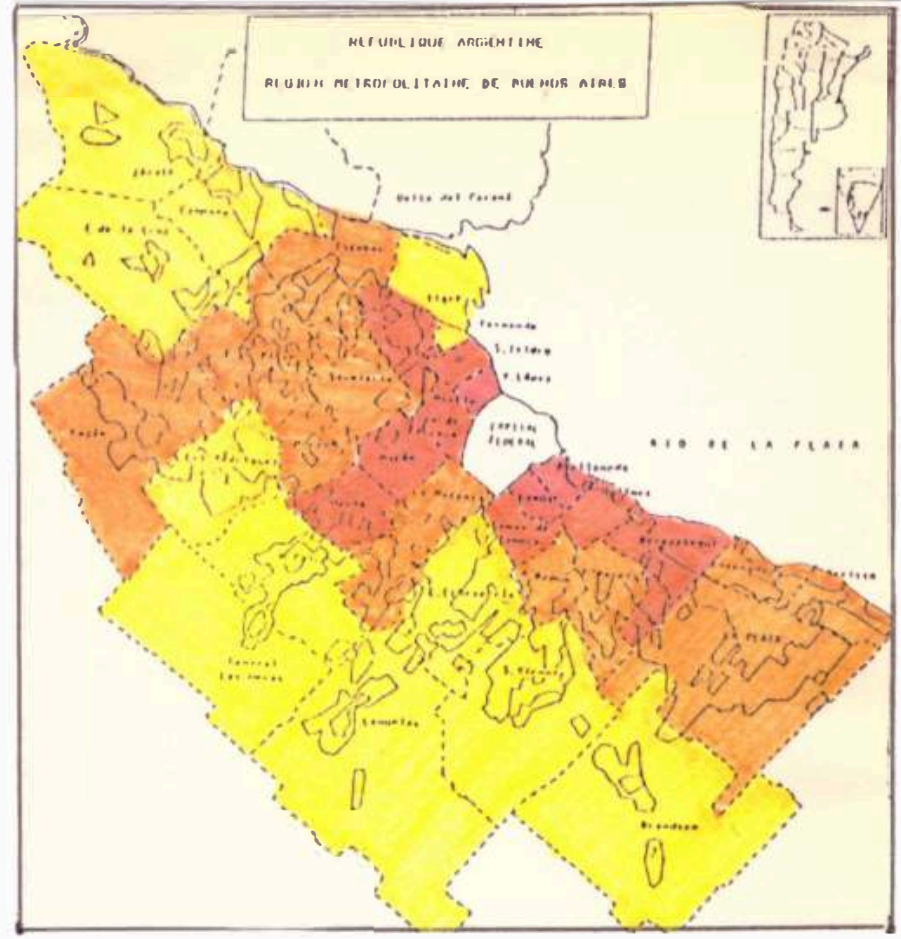

FIGURE 12b

REGION METROPOLITAINE DE BUENOS AIRES

POURCENTAGE D'EXPLOITATIONS DE MOINS 25 HA

PAR RAPPORT AU TOTAL (1937)

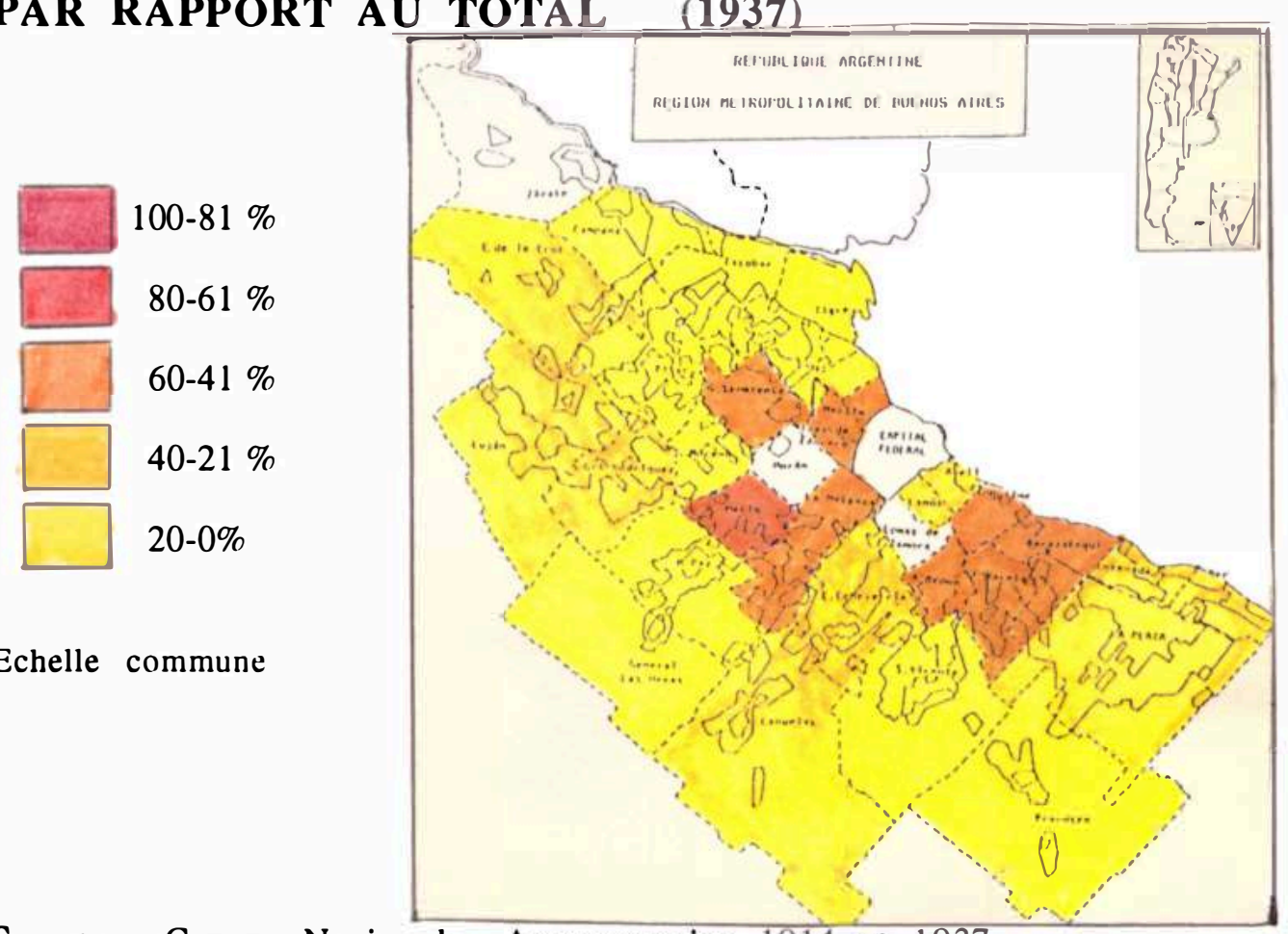

Sources: Censos Nacionales Agropecuarios 1914 et 1937 
FIGURE 13a

REGION METROPOLITANA DE BUENOS AIRES

POURCENTAGE D'EXPLOITATIONS DE MOINS 25 HA

PAR RAPPORT AU TOTAL (1969)

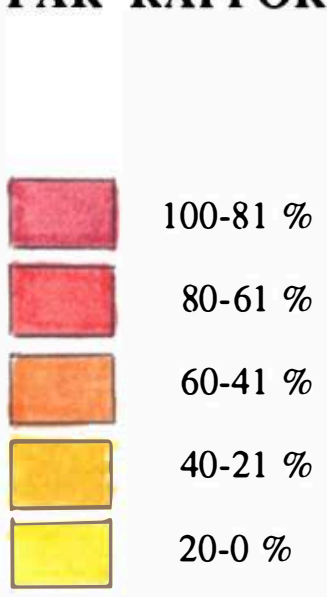

Echelle commune
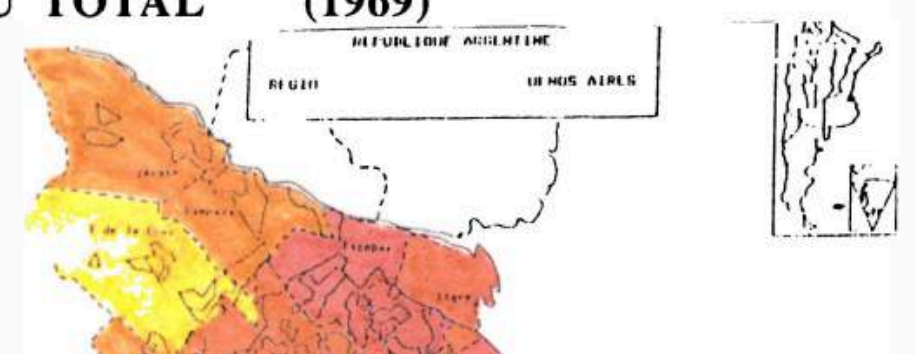

FIGURE 13b

REGION METROPOLITAINE DE BUENOS AIRES

POURCENTAGE D'EXPLOITATIONS DE MOINS 25 HA

PAR RAPPORT AU TOTAL (1988)
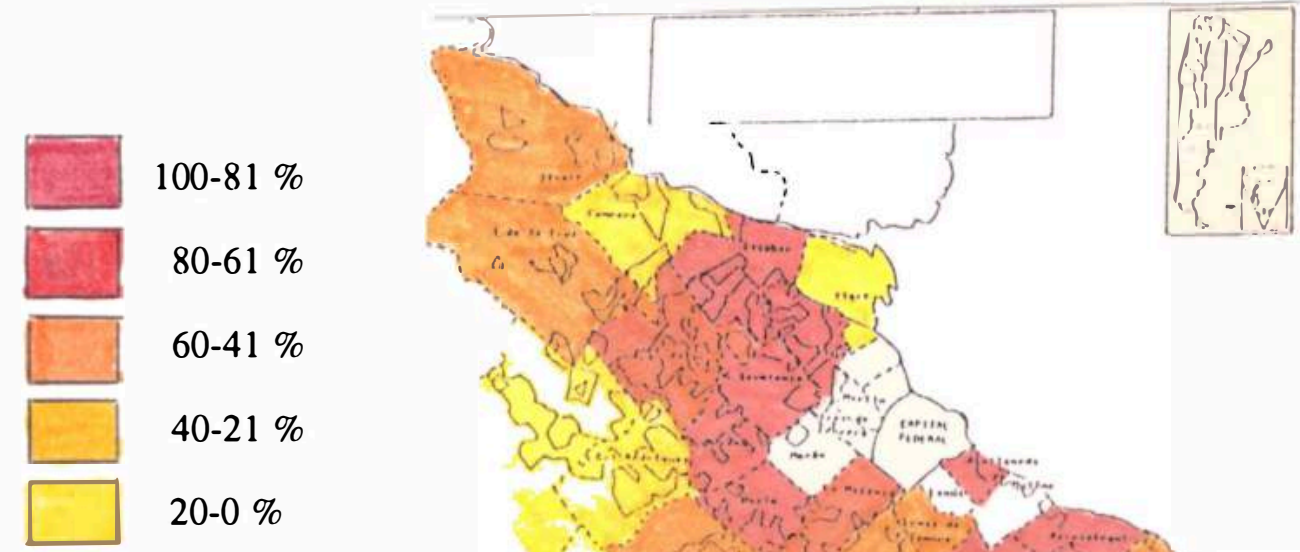

Echelle commune
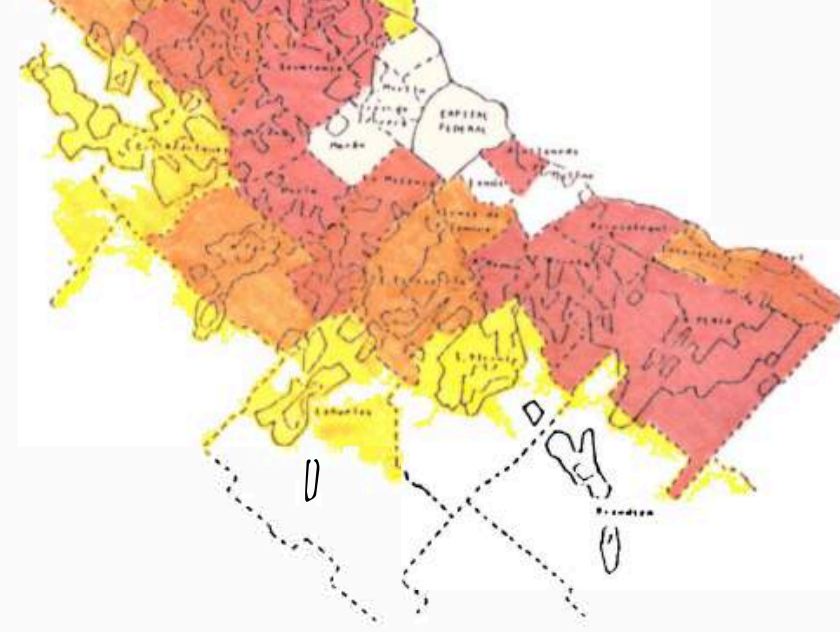

Sources: Censos Nacionales Agropecuarios 1969 et 19 
FIGURE 14a

REGION METROPOLITAINE DE BUENOS AIRES

SUPERFICIE TOTALE DES EXPLOITATIONS DE MOINS 25 HA

(1914)

\begin{tabular}{|c|c}
\hline & 8404 \\
\hline & $6600-5400$ \\
\hline & $3700-2500$ \\
$\square$ & $2300-1500$ \\
$\square$ & $1500-700$ \\
$\square$ & $650-170$ \\
$\square$ & $90-1$
\end{tabular}

Seuls observés

Echelle commune

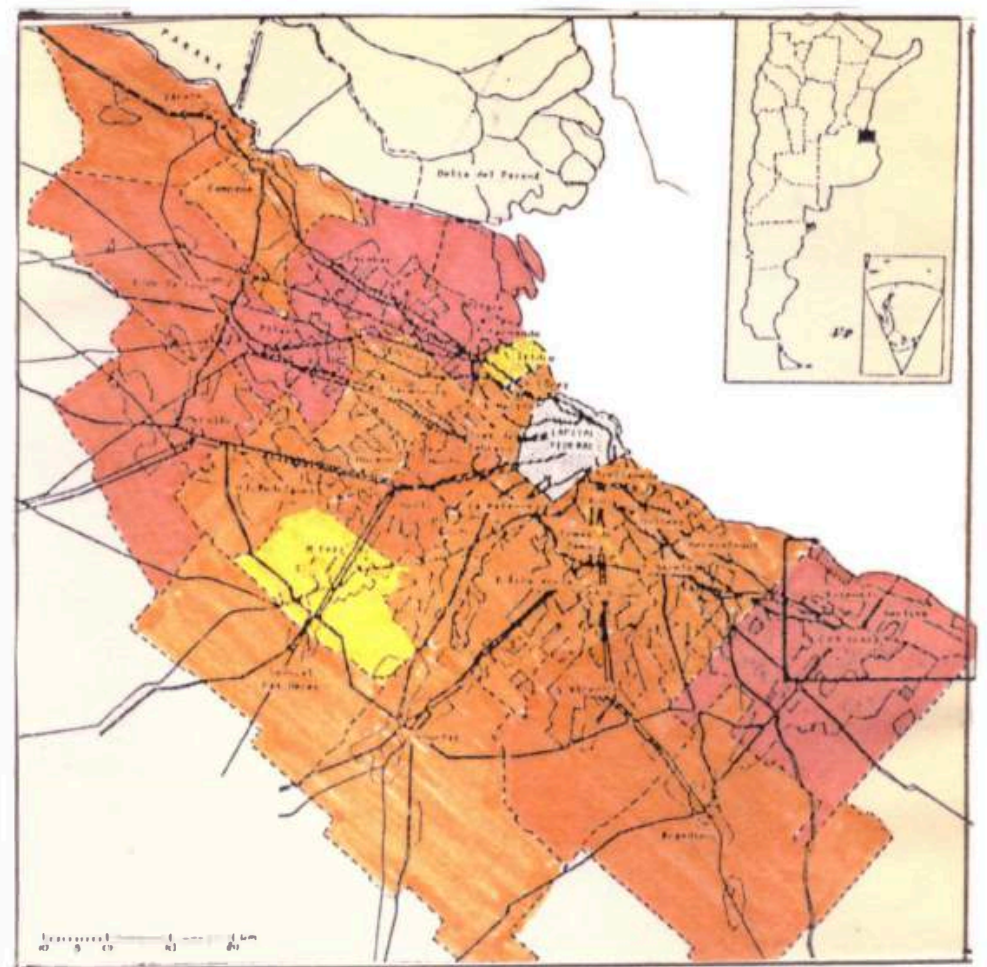

FIGURE 14b

REGION METROPOLITAINE DE BUENOS AIRES

SUPERFICIE TOTALE DES EXPLOITATIONS DE MOINS

25 HA (1969)

$6600-5400$

$3700-2500$

2300-1500

1500-700

$650-170$

90-1

Seuls observés

Echelle commune

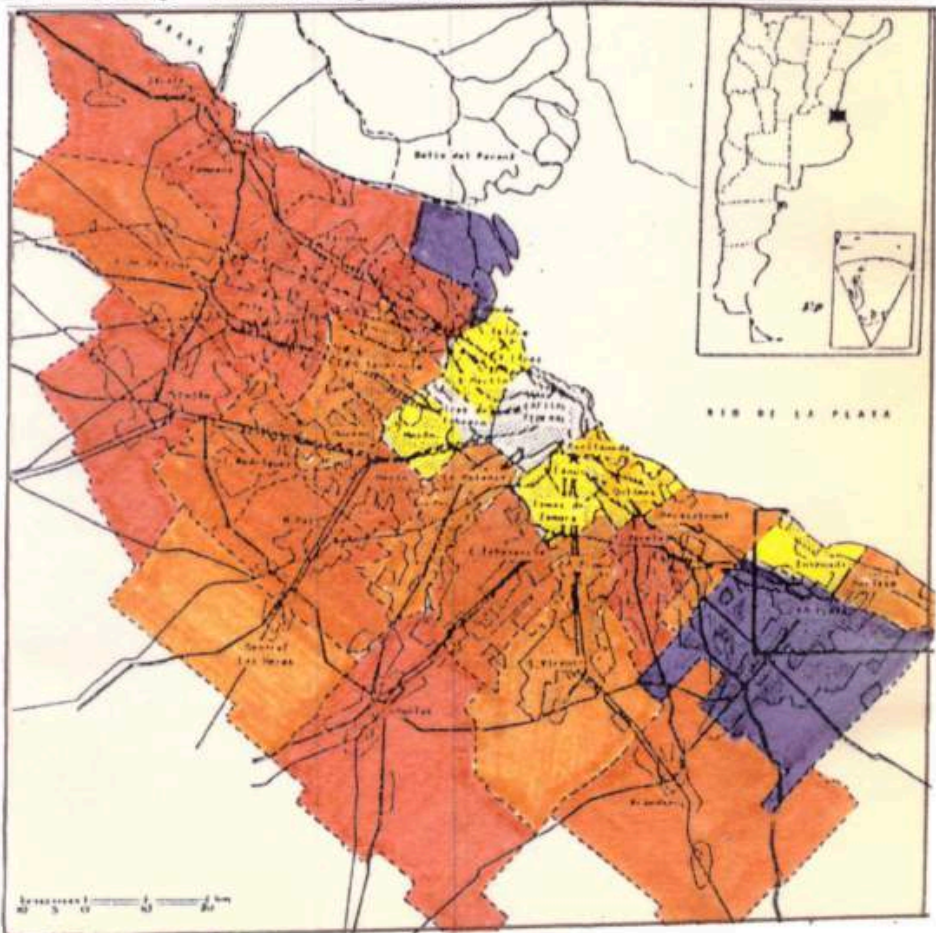

Sources: Censos Nacionales Agropecuarios 1914 et 1969 
FIGURE 15a

REGION METROPOLITAINE DE BUENOS AIRES

PROPORTION DE SUPERFICIE DES PETITES EXPLOITATIONS (1914)

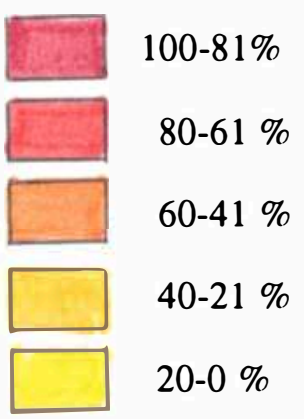

Echelle commune

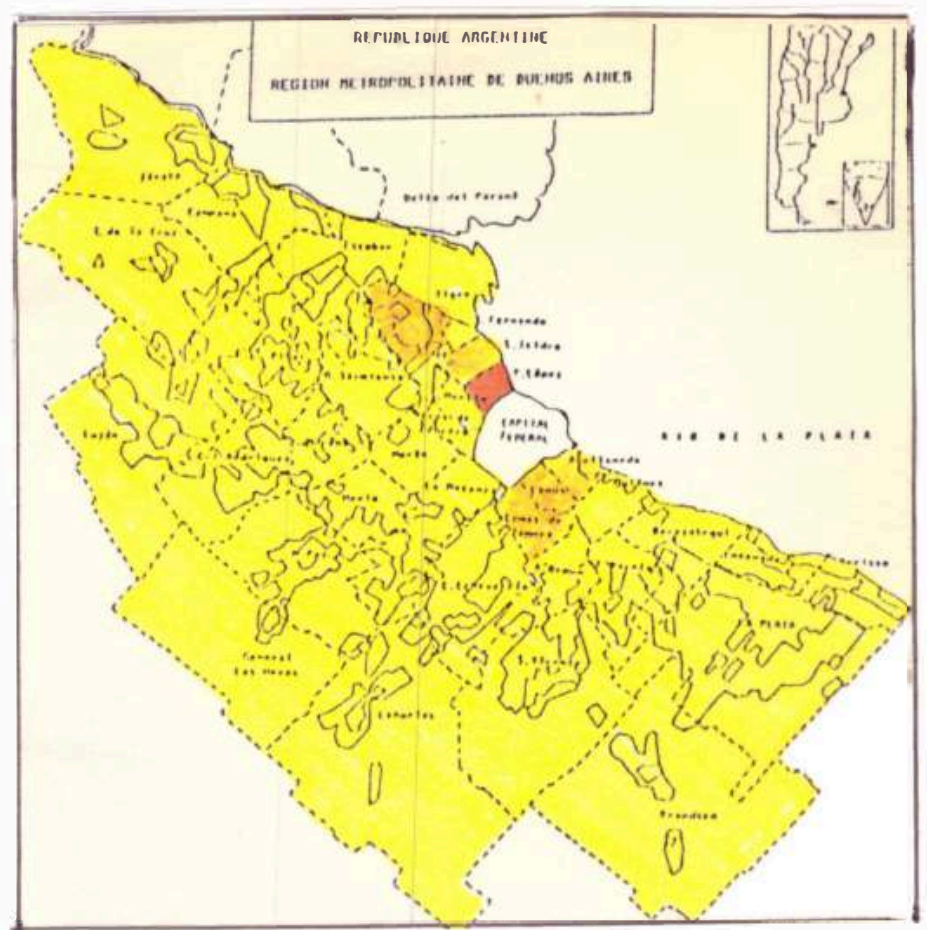

FIGURE 15b

REGION METROPOLITAINE DE BUENOS AIRES

PROPORTION DE SUPERFICIE DES PETITES EXPLOITATIONS

(1969)

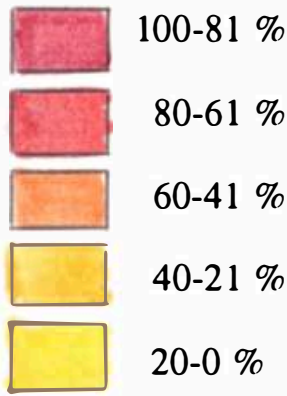

Echelle commune

Sources: Censos Naciona

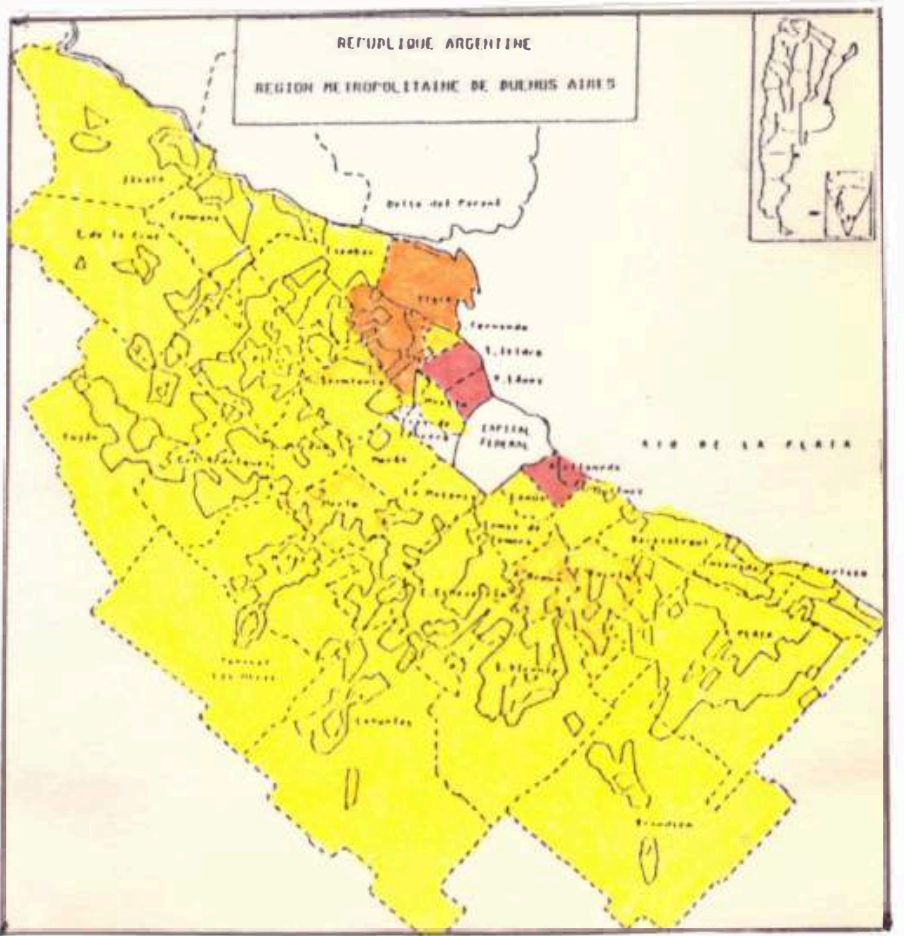


FIGURE 16a

REGION METROPOLITAINE DE BUENOS AIRES

SUPERFICIE TOTALE DES EXPLOITATIONS DE MOINS 25 HA ( 1988 )

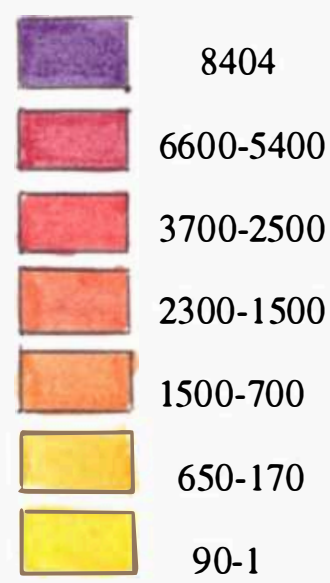

Echelle commune Seuls observés

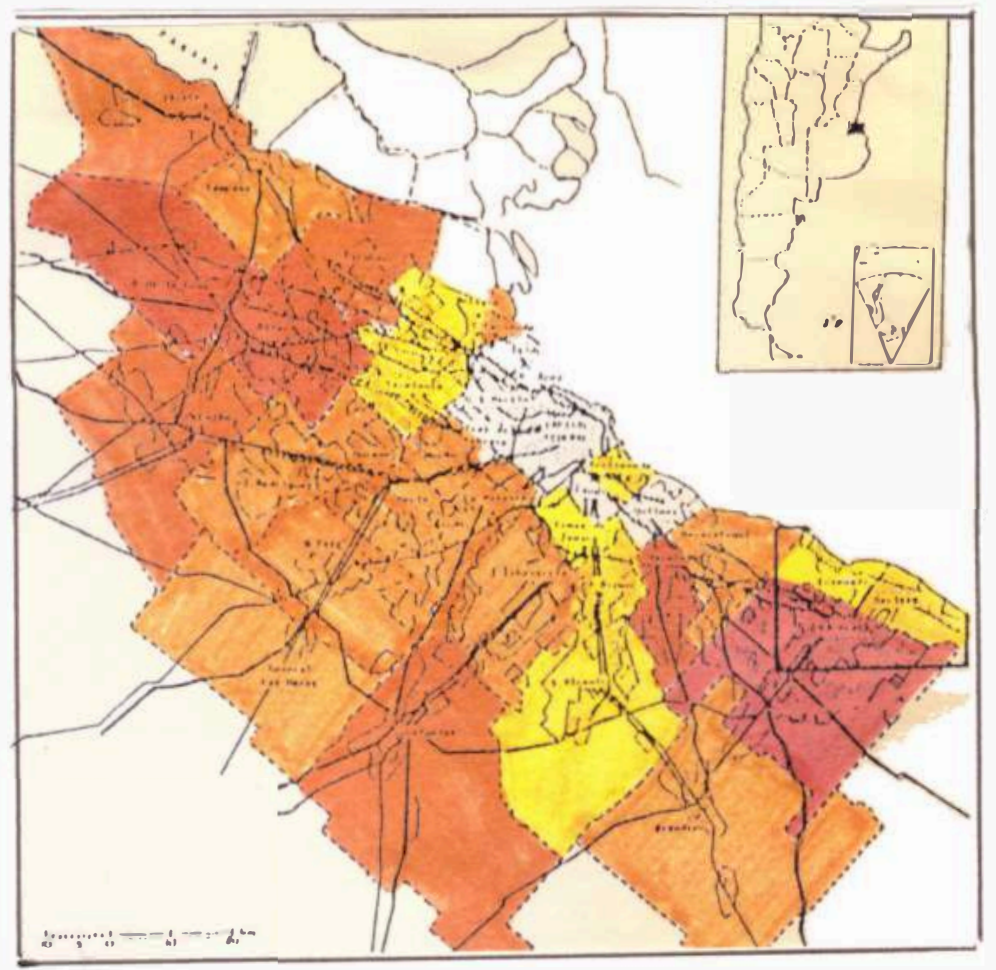

FIGURE 16b

REGION METROPOLITAINE DE BUENOS AIRES

PROPORTION DE SUPERFICIE DES PETITS EXPLOITATIONS ( 1988 )

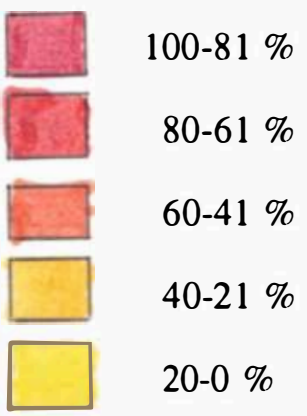

Echelle commune

Seuls observés

Source: Censo Nacional Agropecuario

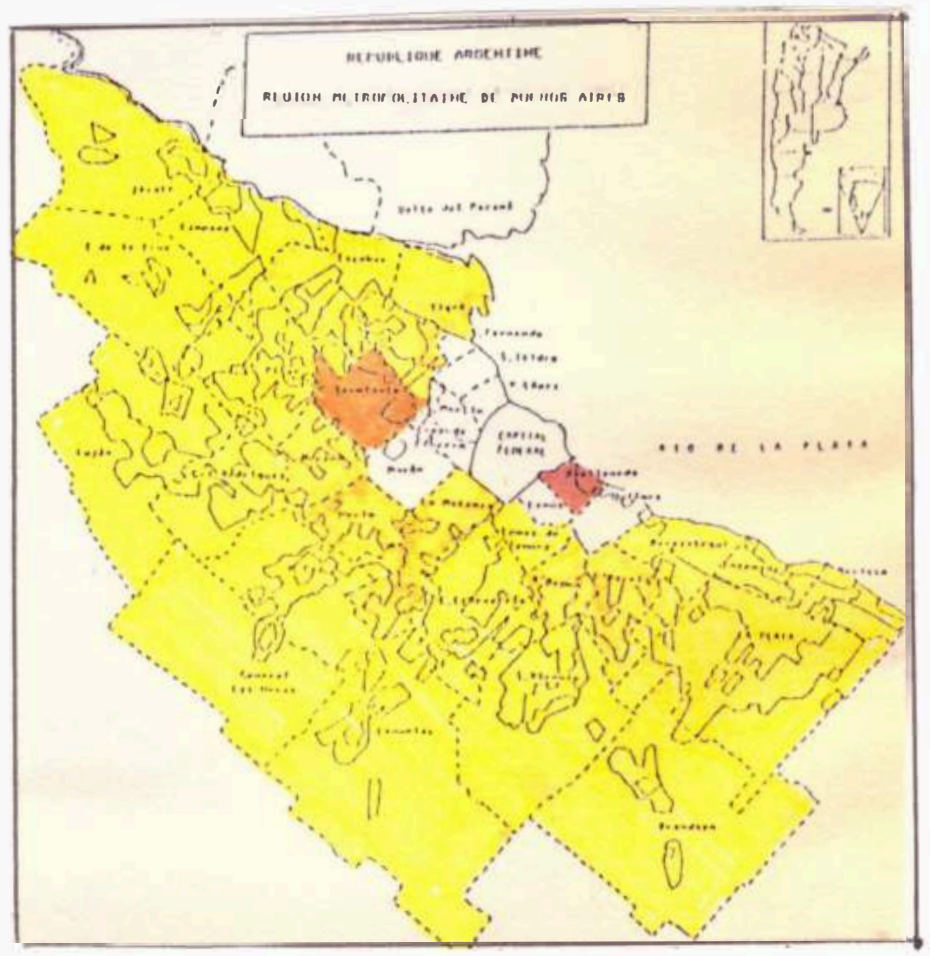


FIGURE 17

LE SUD-EST DE LA REGION METROPOLITAINE DE BUENOS AIRES

LES MICRO-REGIONS DE ENSENADA-BERISSO

\section{ET DE INGENIERO ALLAN-EL PATO-EL PELIGRO}

On remarque les espaces urbains dominants dans chaque unité territoriale: en bleu, plus de $50 \%$ d'espace occupé; en bleu ciel, moins de $50 \%$ d'espace occupé. Elaboration personnelle.

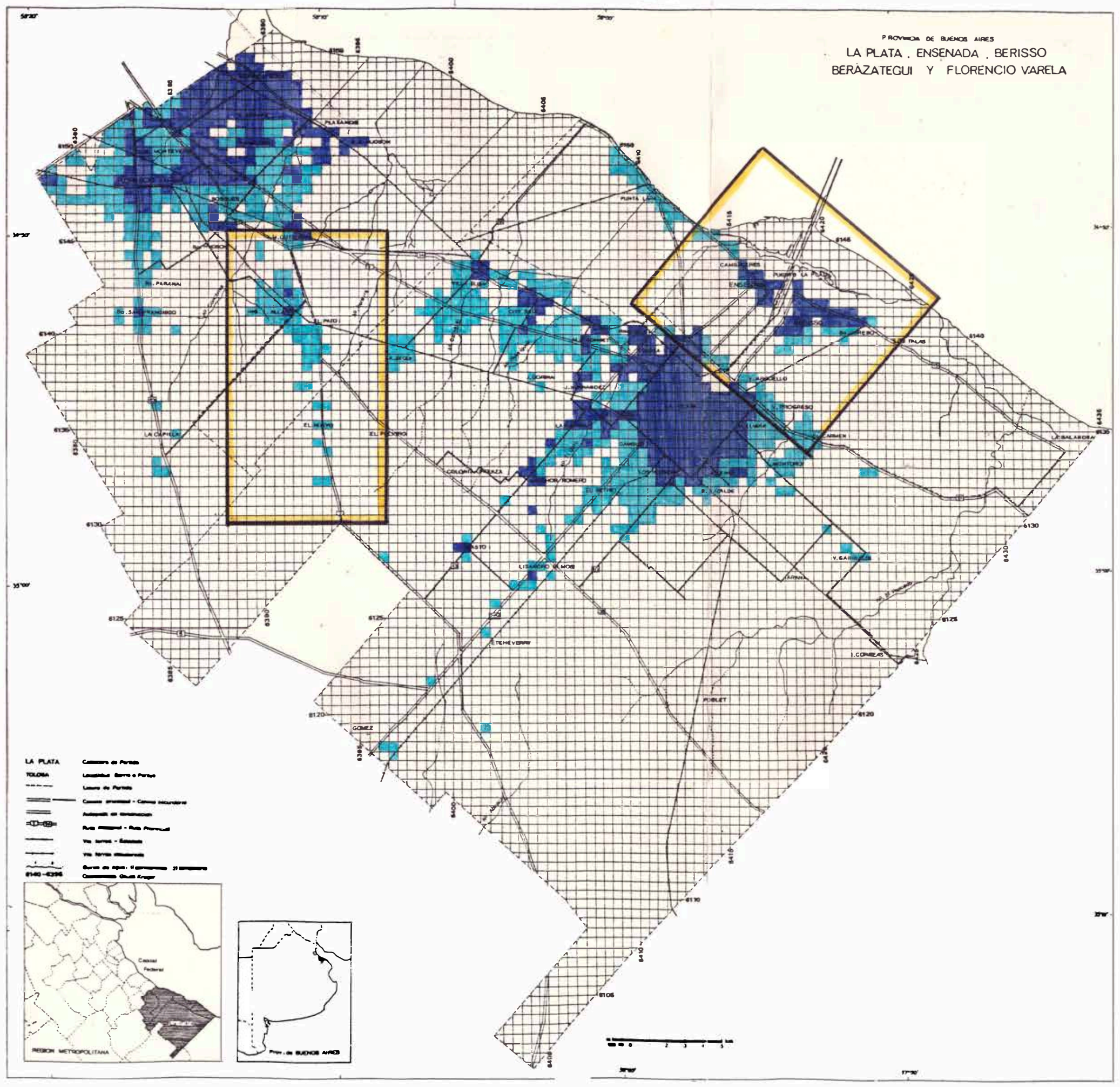


FIGURE 18

LE SUD-EST DE LA REGION METROPOLITAINE DE BUENOS AIRES BERISSO, ENSENADA, LA PLATA, F.VARELA ET BERAZATEGUI

\section{ALTITUDES DOMINANTES DU RELIEF PAR UNITE TERRITORIALE}
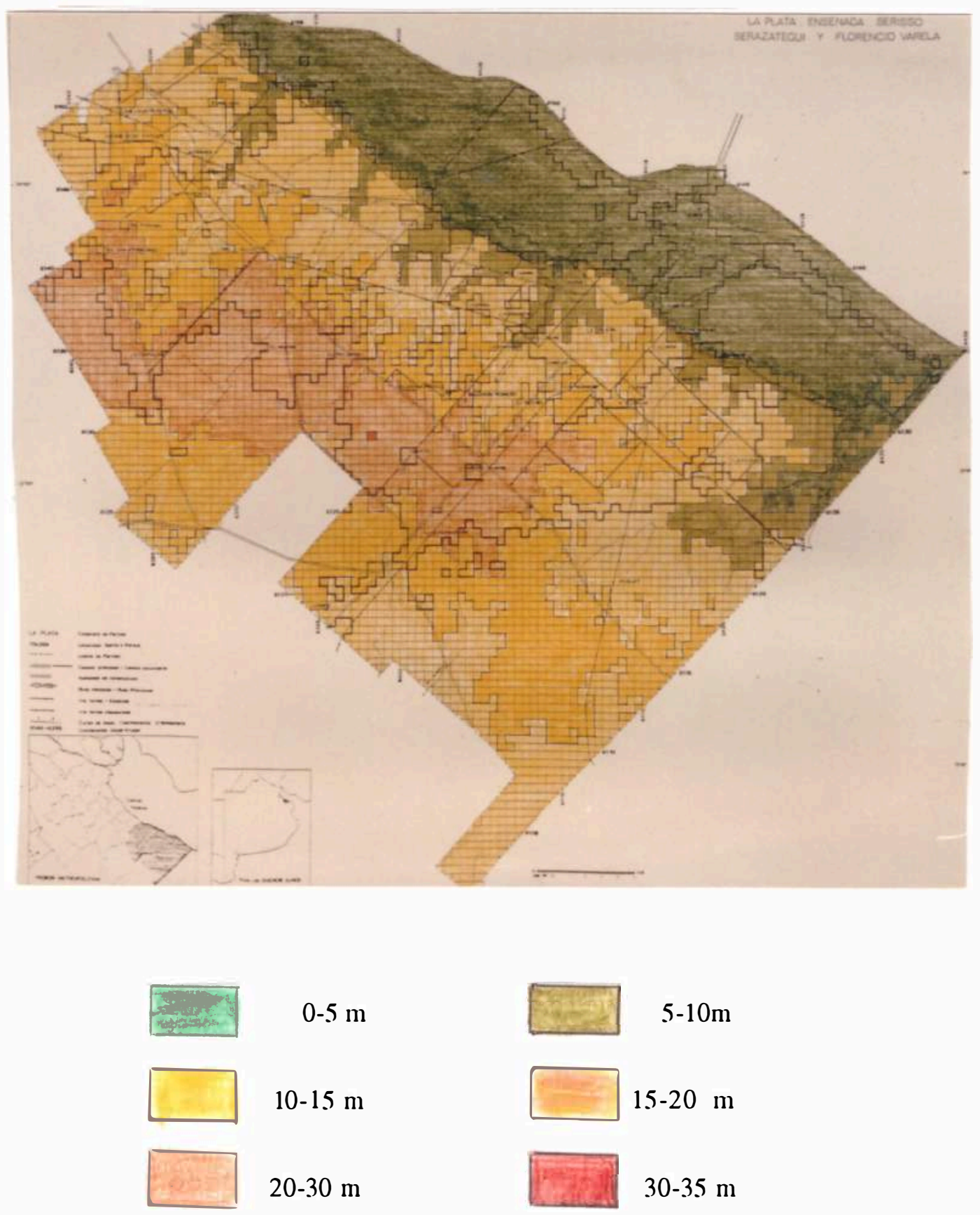

Source: Cartes Topographiques Institut Géographique Militaire Argentin. "La Plata" et "Villa Elisa" 


\section{3 - LA MICRO-REGION \\ D'ENSENADA ET BERISSO}

La micro-région d'Ensenada et Berisso, de $112 \mathrm{~km} 2$ et 115.200 habitants en 1991, est l'un des rares scénarios du territoire argentin où il est possible de déceler des manifestations des processus socioéconomiques et politiques qui ont eu lieu durant des quatre siècles. Depuis son appartenance à la Vice-Royauté du Pérou vers la fin du XVIème siècle jusqu'aux premières formes d'insertion dans le nouvel ordre économique en vigueur.

Dans ce contexte, les mutations industrielles ont joué un rôle très important. Il est possible d'identifier toutes les périodes d'industrialisation du territoire argentin, depuis le "saladero" de 1798, jusqu'aux Pôles Pétrochimique et Informatique actuels.

L'Argentine n'enregistre pas de situations tellement évidentes d'un processus d'organisation territoriale à échelle micro-régionale, comme celles d'Ensenada et Berisso. D'autres analogies possibles ne constituent pas les exemples des caractéristiques analysées. Pourquoi ? Parce que dans le cas de Buenos Aires, il s'agit d'une autre échelle, où s'articulent des formes de consolidation et de perpétuation de pouvoirs économiques, politiques et culturels complexes et variés. Dans d'autres cas -Mendoza, Santiago del Estero, Santa Fé, Córdoba- la séquence de processus globaux s'altère ou s'interrompt durant certains périodes.

Dans les actuels Partidos de Ensenada et Berisso se reconnaissent les manifestations suivantes des processus globaux incriminés:

1 La formation territoriale comme appendice d'une Vice-Royauté Pérou- avec siège vers l'océan Pacifique (1580-1730): Les "suertes de estancia" et l'élevage "cimarrón" ou presque sauvage. (FIGURE 19a-page 80 )

2 L'origine d'une autre Vice-Royauté -Río de la Plata en 1776- à partir de la consolidation politique et le développement du pouvoir 
portuaire-commercial vers l'océan Atlantique à Buenos Aires (17301790): Le "Fuerte de Ensenada de Barragán". (FIGURE 19b)

3 La mise en valeur des "pampas" à partir de l'élevage destiné à l'exportation de "tasajo" -viande salée- (1790-1890): Les "saladeros", la vinculation à Buenos Aires et la naissance de Berisso. (FIGURE 19c)

4 L'inclusion dans le modèle "agro-exportador" à travers la production de viandes congelées (1890-1930): Le Port de La Plata, l'immigration européenne et les frigorifiques. (FIGURE 19d)

5 Le développement industriel durant la période substitutive d'importations et la convivialité avec le modèle "agro-exportateur" (1930-1950): La raffinerie "La Plata" de YPF et la survivance du frigorifique. (FIGURE 20a)

6 L'expansion du marché intérieur et la diversification industrielle (1950-1975): La consolidation urbaine et la diversification industrielle. (FIGURE 20b)

7 La diminution du marché intérieur, la désindustrialisation et la reconversion économique actuelle (1975-1992): Les Pôles Pétrochimique et Informatique, la Zone Franche du Port La Plata et les "villes-dortoir".

\section{Le milieu naturel}

Ensenada et Berisso se situent sur la plaine côtière dite "Planicie Costera" Il s'agit d'une frange de terrain presque plane, qui s'étend sur la rive droite du Río de la Plata, avec une largeur comprise entre 5 et $8,5 \mathrm{~km}$ et des hauteurs entre 1,5 et $2,2 \mathrm{~m}$ dans la plupart des cas. (FIGURE 18, 20d et 21)

Cette plaine est formée de dépressions et de zones positives qui ne dépassent pas $5 \mathrm{~m}$ au-dessus du niveau de la mer; 90\% du territoire est au dessous de $3 \mathrm{~m}$ (Fidalgo;1983:272). Les villes d'Ensenada et Berisso se trouvent dans une douce élévation du terrain en forme de ceinture dit "albardón" de $3 \mathrm{~m}$ de hauteur et $18 \mathrm{~km} 2$ au maximum. Le sol de la plaine marécageuse est "gley salin-alcalin" (Cappanini; 1966) (FIGURE $20 \mathrm{~d}$ ) 
Les plus grands préjudices dus aux inondations, ne sont pas en relation avec le vent du sud-est, dit "Sudestada", mais avec des difficultés de drainage par l'inefficacité des réseaux de bondes pluviales. A cette situation s'ajoute la pente naturelle presque nulle.(Bozzano;1989:12)

Les crues causées par la "Sudestada" sont sporadiques, mais catastrophiques. On remarque celles de 1923 de $3,69 \mathrm{~m} ; 1940$ de 4,02 m et 1989 de $3,64 \mathrm{~m}$.

Le milieu a été modifié par des événements naturels et humains. Les courants de dérive littoraux ont transformé la vieille "Ensenada de Barragán" en presqu'île, aujourd'hui connue comme "Isla Santiago" La nature alluviale du sol a été le support de développement de nombreuses exploitations maraîchères à "Los Talas" et "Isla Paulino" (FIGURE 20d)

Les modifications les plus fortes du milieu ont été provoquées par la construction du Port La Plata vers 1886: un dock de 4,5 km de longueur. Le remplissage de terrain pour les industries commence vers la decennie de 1920 avec la "Destilería La Plata" de YPF; il continue jusqu'à nos jours.

Cette altération du milieu physique donne lieu à des changements des conditions de drainage, déjà modifiées avec la rectification des ruisseaux sans pente dites "divagantes" transformés en canaux. L'égouttement superficiel tout au long de l'aire industrielle n'enregistre aucune oeuvre de canalisation. Les interstices non-industriels sont des "dépôts d'eau" qui restent inondés durant des périodes considérables. Le cas extrême est celui du "Parque Martín Rodríguez" Il s'agit de 200 hectares boisés qui connaissent un processus de déterioration par l'excès d'humidité.

\section{TRANSFORMATIONS TERRITORIALES \\ ET MUTATIONS PRODUCTIVES. \\ UN ESSAI DE PERIODISATION}

\section{LES "SUERTES DE ESTANCIA" (1580-1730)}

En 1580 Juan de Garay établit une répartition de terres au nord et au sud de Buenos Aires sur la rive du Plata. Dans les terres plus fertiles de meilleur drainage -le nord- il assigne des "tierras de pan llevar" à des 
fins agricoles, tandis qu'au sud, dans des sols moins fertiles fixe 29 "suertes de estancia" pour l'élevage. (FIGURE 2-page 36)

Celles-ci s'étendaient entre "Punta Gaytán" -actuellement Wilde, Avellaneda- et la Vallée du Santa Ana -actuellement "Cañada de Arregui, Magdalena-. Chaque propriété avait $2600 \mathrm{~m}$ de front sur la petite ravine, dit "barranca costera" $\mathrm{La}$ zone marécageuse connue comme "Planicie Costera" n'a pas été atribuée: là se trouvent les actuelles Ensenada et Berisso.

En 1618 on assigne ces terres à Bartolomé López qui commence à les exploiter pour l'élevage bovin, de la même façon que dans le passé avec les "suertes de estancia" En 1629, ces terres sont acquises par Antonio Gutiérrez Barragán qui déclare l'existence de 2000 vaches dans la zone marécageuse.(Bozzano et Vives; 1982:65)

Le développement de l'élevage, associé aux activités portuaires et commerciales, est décisif pour le transfert du siège de la "Gobernación del Río de la Plata" d'Asunción du Paraguay à Buenos Aires vers 1617. Par les "Leyes de Indias", on établit l'usage commun de pelouse, arbres et sources d'eau, depuis 1667.

Malgré la fragilité du milieu et la nature extensive de l'élevage, il y a un rapport "territoire-production" étroit. Même actuellement, presque quatre siècles après, il s'agit de la seule activité possible sur ce type de sol avec une très faible charge d'animaux par hectare. (FIGURE 19a-p. 80)

\section{2 - LE "FUERTE DE LA ENSENADA DE BARRAGAN" (1730-1790)}

Sur la côte des terres de Gutiérrez Barragán il existait une crique dit "ensenada" avec les meilleurs conditions naturelles de toute la rive droite du Plata pour le mouillage de navires

Elle constituait vers les XVII et XVIIIème siècles une barrage naturelle avec un ensemble d'îles de sable et de limon á partir de la "Punta del Monte Santiago" Leur dynamique et le caractère alluviale de leur formation deviendraient plus tard les facteurs de leur remplissage et de leur future disparition en tant que crique ou petite baie. Vers le XIXème siècle, elle était devenue l'embouchure du "Río Santiago" (FIGURE 19b-p.80)

En 1727, Juan A.Guerrero, un habile pilote du Plata parle des excellentes conditions naturelles de l'"Ensenada" Un peu plus tard, le 
Gouvernement de Buenos Aires informe de cette nouvelle: en 1731 se construit la forteresse, dite "Batería de l'Ensenada de Barragán", pour la défense et pour éviter le commerce illicite des portugais, localisés à 40 km (Colonia del Sacramento, actuel Uruguay).

Lors de la création de la Vice-Royauté du Río de la Plata, matérialisée en 1776, Ensenada est un site de défense contre les invasions du Brésil et d'outre-mer.

Vers 1778, Ensenada est un hameau habité par 22 familles. Selon William Rogind, il était "un presque village ayant une activité assez importante" A cause d'une guerre entre l'Espagne et l'Angleterre, la Vice-Royauté renforce la "Batería"

La continuité du même rapport "territoire-production" persiste jusqu'à la fin du XVIIIème siècle. Durant cette période se consolide un territoire fragile et dépeuplé, par le déploiement d'une activité manufacturière très importante: la préparation de viande salée pour l'exportation.

\section{3 - LES "SALADEROS", LA CONNEXION A BUENOS AIRES ET LA NAISSANCE DE BERISSO (1790-1890)}

Le fort développement d'Ensenada est relié à la nécessité de consolider politiquement Buenos Aires et à la possibilité de commercialiser des produits agro-pastoraux à l'extérieur.

En 1798, Agustín Wright installe le premier "saladero" du territoire argentin. Il constitue le début d'une mutation productive très importante pour l'économie argentine: l'exportation de viande salée depuis la "pampa" à travers les ports du Riachuelo à Buenos Aires et d'Ensenada.

Durant 1806, la forteresse résiste aux "Invasions Anglaises" et l'année suivante reste une semaine occupée par les britanniques. Il s'agit des premières manifestations de l'intêret anglais pour avoir le contrôle économique de la production de viande. En 1810, Robert Staples et John McNeil, des commerçants d'origine britannique, installent le deuxième "saladero" à Ensenada. Un peu plus tard ils s'associent à Pedro Trapani. Cinq ans plus tard, le port est habilité pour des bateaux d'outre-mer. (FIGURE 19c)

L'inauguration du "Chemin Blanc" en 1821 signifie la vinculation à Buenos Aires à travers le "Chemin Royal" 
Le combat de "Monte Santiago", en 1827, dans le cadre de la Guerre avec le Brésil, est déterminante du transfert du "saladero" de Staples à "La Boca" (Buenos Aires). La lutte entre la fonction défensive et la fonction économique est assez forte: Ensenada est un excelent port, mais il y a aussi des constructions de défense importantes et une situation stratégique avantageuse pour Buenos Aires.

L'orientation des luttes vers l'intérieur du pays -entre "Unitarios" et "Federales"- déplace le site de confrontation. Ensenada donc, se consolide comme centre de production et de commercialisation de viande salée. Pendant la décennie de 1870 est inauguré le chemin de fer à Buenos Aires et la zone compte 223 habitants dans le hameau et 2917 à la campagne; une bonne proportion est constituée des travailleurs des "saladeros"

Les 66 établissements de la zone, dits "estancias", ont presque un million d'ovins métis, plus un nombre moins important de bovins et équins. Le port d'Ensenada est le premier exportateur argentin de viande salée, dit "tasajo" -11.000 tn- en destination des Antilles et du Brésil.

L'épidémie de fièvre-jaune en 1871, localisée dans les quartiers portuaires de Buenos Aires, est l'autre déterminante du transfert des "saladeros" à Ensenada et ses environs. Ainsi, cette année-là avec l'installation du "saladero San Juan" de Juan Berisso, naît un petit hameau non planifié à $3 \mathrm{~km}$ de Ensenada. Ce sera la ville de Berisso. La même année, à Ensenada, Antonio Cambaceres, inaugure le "saladero Tres de Febrero" Les deux occupaient 2000 ouvriers dans les périodes de plus grand travail. Juan Berisso même installe un peu plus tard le "saladero San Luis"

Parallèlement, c'est une époque d'essais pour améliorer et approfondir la technique de préparation de viande. On vérifie une tendance de changement de consommation de la viande de mouton pour la viande de vache.

Vers la decennie de 1880, Ensenada compte 3300 habitants et Berisso 1000. Il s'agit d'une époque de grandes transformations territoriales à cause de décisions politiques très importantes. En 1882 est fondée la ville de La Plata, à $4 \mathrm{~km}$ de Ensenada et Berisso. En 1883, on commence les oeuvres de construction d'un grand port qui séparerait physiquement les deux villages étudiés. 
Lamutation productive -manufacture primaire du "saladero"- est la cause d'importantes transformations territoriales: la naissance d'un village, Berisso, et la croissance d'un autre, Ensenada.

Si la diffusion généralisée tardive d'une procédure de traitement primaire pour la viande détermine de fortes transformations territoriales vers le dernier tiers du siècle; le scénario est l'un des privilégiés dans la réception d'une mutation productive innovatrice qui permet de faire des exportations de viande en froid. Il s'agit de l'industrie frigorifique.

\section{4 - LE PORT LA PLATA, L'IMMIGRATION EUROPEENNE ET LES FRIGORIFIQUES (1890-1930)}

Cette technologie du froid pour la viande, testée en France, s'intègre quel que soit le territoire, avec le seul objectif d'assurer la consécution d'un circuit économique.

Les "pampas", avec trois siècles d'élevage et un récent raffinage d'espèces bovines constitue l'un des territoires choisis pour cette mutation productive.

La décision politique de localiser la nouvelle capitale provinciale proche de Buenos Aires -56 km- dans un territoire de 307.500 km2 aura des connotations négatives pour leur développement ultérieur. La ville de La Plata a une croissance explosive: 10.407 habitants en 1884 et 65.610 en 1890 dont 57,7\% étrangers. (FIGURE 19d-p.80)

Six mois avant la fondation de La Plata, on signe un accord entre les gouvernements national et provincial pour la construction d'un port de 21 pieds de calaison, profondeur adéquate pour les bateaux de l'époque.

L'ingénieur hollandais J.Waldorp est nommé directeur technique des oeuvres qui commencent en 1883. Quelques milliers d'ouvriers creusent à la pelle un canal de $4500 \mathrm{~m}$ de longueur sur $50 \mathrm{~m}$ de large. Il existe, en projet, un antécedent de transformation territoriale et de mutation productive notable et peu connue: la "Compagnie de Quais, Canal et Village de Punta Lara" enregistre une étude de Waldorp de 1889, sur la réalisation de polders dans la zone marécageuse, avec dix villages et des zones reservées pour l'industrie.

L'ouverture du Port La Plata en 1890 est aussi le début d'une concurrence inégale avec le Port Madero à Buenos Aires. Malgré sa 
petite taille et sa calaison insuffisante, il s'imposera très vite sur celui de La Plata. Les oeuvres du "Puerto Nuevo" à Buenos Aires, d'une part, et la fin de la juridiction provinciale sur le port et le chemin de fer en 1904, donc l'augmentation des coûts de frets terrestres, d'une autre, sont les évidences du triomphe du centralisme de Buenos Aires. (FIGURE 19d)

La période 1890-1896 est celle d'une croissance majeure, en ce qui concerne le mouvement d'outre-mer. Après, le port diminue d'importance. Les exportations de vaches et de viande et les importations de combustibles continuent jusqu'aux années trente.

L'interdiction d'entrée de bétail sur pied dans les ports anglais vers 1890 est le début d'une mutation productive. L'entreprise "Zavalla y Compañía" installe en 1894 un embarcadère de bétails et en 1900 obtient une concession de quarante ans pour l'installation à Berisso d'un établissement de congélation de viandes et conserves alimentaires, avec l'exemption d'impôts provinciaux et municipaux.

Zavalla paye les droits du contrat à l'entreprise anglaise "Cold Storage" En 1902 on construit très vite leurs bâtiments. En 1904 est inauguré l'abattoir avec une capacité de 450 bovins et de 3000 ovins par jour.

En 1908, le frigorifique commence à être exploité par des capitaux nord-américains, en employant 3000 ouvriers. En 1916 leur nom est changé en "Compañía Swift-La Plata": en huit ans il multiplie par trois leur capacité d'emmagasinage.

En 1915 s'ouvre à Berisso la "Sociedad Anónima Frigorífica Armour-La Plata" d'origine nord-américaine. Vers 1932 on atteint la majeure capacité d'abattre: 4000 ovins et 2500 bovins, avec 13.000 ouvriers dans les deux frigorifiques. On produisait du "chilled-beef", du "frozenbeef", des graisses, des beurres, des huiles et des suifs. Des établissements derivés naissent dans les environs tanneries et graisseries.

Latransformation territoriale donnée par ladite mutation productive est remarquable. Elle concerne l'expansion urbaine de Berisso avec l'arrivée de nombreux immigrants. Durant cette période s'ajoute le début des activités agricoles intensives à la zone alluviale, avec des immigrants européens aussi. "Los Talas", "Isla Paulino" et "Isla Santiago" se développent. C'est une période de formation de petites exploitations forestières, fruitières et horticoles, la plupart dans le commune de Berisso. 
Vers 1909, le village de Berisso aurait 2553 habitantes, Los Talas 970, et les îles Paulino et Santiago, 873 habitants. Les propriétaires et les travailleurs étaient étrangers, $85 \%$ et $59 \%$, respectivemment. La croissance démographique est explosive. En 1914, Ensenada enregistre 13779 habitants, dont 38\% étrangers; Berisso, 8847 et 59\% étrangers; et la zone alluviale, 3042 habitants. Les groupes identifiés sont nombreux, principalement des Italiens et Espagnols, mais aussi des Arabes -surtout de Syrie et du Liban-, des Lithuaniens, des Ukrainiens, des Yougoslaves, des Grecs, des Bulgares, des Polonais et d'autres nationalités.

La multiplication par sept du nombre d'habitants à Berisso en huit ans est due exclusivement au développement de l'industrie frigorifique. Les transformations territoriales peuvent s'identifier à travers l'évolution de la subdivision du sol et les caractéristiques du logement.

Vers 1916, la ville d'Ensenada comportait 255 îlots, tandis que Berisso en avait 156. En 1947, ce nombre avait atteint 289 et 560, respectivement. (FIGURE 25-p.85)

La petite ravine de Berisso dit "albardón" est plus étendue que celle d'Ensenada. La prolifération de lotissements en damier n'est pas de même nature que le processus d'occupation de la population. La plupart des lots reste inoccupée durant deux ou trois décennies. La zone prochaine aux frigorifiques se couvre de typiques logements de "zinc" de basse qualité. Les conditions de vie sont extrêmement précaires. C'est l'époque connue comme "chambre-chaude" où les ouvriers alternaient repos et travail dans les mêmes lieux.

Offre d'emploi. Offre de terre urbaine sans services. Manque de logement. Un décalage très fort dans le processus de transformation territoriale.

\section{LA RAFFineRIE LA PLATA DE YPF ET LA SURVIVANCE DU FRIGORIFIQUE (1930-1950)}

Les années postérieures à la découverte du pétrole dans le territoire argentin en 1907 sont des essais pour obtenir une valeur ajoutée optimale par son raffinage.

Vers 1923, un an après sa création, YPF-Gisements Pétroliers de l'Etat prend possession d'une frange stratégique de terres fiscales, originairement destinées à la réalisation des oeuvrages du Port La Plata. (FIGURE 20a) 
A partir d'un projet de la "Betlehem Steel Company", on inaugure en 1925 la "Destilería La Plata", la première, la plus grande et la plus moderne de l'Argentine. Cette mutation industrielle déclanchera le développement local jusqu'à maintenant.

Leur industrie de raffinage primaire commence avec une production de $300 \mathrm{~m} 3 /$ jour, une capacité de $2000 \mathrm{~m} 3 /$ jour, dans un terrain de 80 ha et avec 300 ouvriers. Les manifestations centrales de son développement et de son consolidation sont l'incorporation de nouvelles technologies, l'expansion en superficie, la diversification productive, l'augmentation du nombre d'employés et l'utilisation croissante du port pour le cabotage pétrolier. Ce progrès est évident: actuellement, la capacité de production est de $50000 \mathrm{~m} 3 /$ jour.

Durant cette période, le port se spécialise dans le pétrolier. Entre 1925 et 1965 , le mouvement de cabotage passe de 300.000 tonnes à 4,5 millions tonnes. La plupart de ces entrées et sorties est constituée par du pétrole brut et ses dérivés.(Maselis; 1982:207)

Contrairement à l'information la plus répandue, le Port La Plata fonctionne aussi comme port d'outre-mer pour la production frigorifique. Entre 1930 et 1950, "Swift" et "Armour" emploient de 10 à 13 mille personnes sans interruption, qui abattent 10.000 têtes par jour.

Le développement d'autres marchés de production et la technologie ankylosée sont deux facteurs qui expliquent leur déclin. Après s'ajouteront les politiques protectionnistes des pays du nord et plus de rigueur en ce qui concerne le contrôle sanitaire.

L'expansion urbaine d'Ensenada et Berisso trouve des limites naturelles. Les nappes phréatiques se situent à moins de $2 \mathrm{~m}$ de profondeur sur le doux "albardón" où se localisent les deux villes. Au delà, les zones marécageuses du "Bañado" et alluvionnaire de l'île Santiago, avec des niveaux phréatiques presque superficiels ne permettent pas l'établissement de la population. (FIGURE 20d)

La zone marécageuse reste dépeuplée. La zone alluviale, boisée et cultivée de fruits et de légumes, avec des logements du type palafittes à cause des inondations périodiques. Le contact "albardón-zone alluviale" reçoit une mutation productive qui contribuirait à la dégradation du milieu: l'exploitation de "conchilla", des restes géologiquement récents de coquilles calcaires. Les excavations alternent, et font disparaître très souvent, les exploitations agricoles à "Los Talas" 
Le début de cette période marque la suprématie de Berisso sur Ensenada. Vers 1940 on estime de 28 à 30 mille et de 20 à 22 mille habitants, respectivement, sans compter les quartiers des communes ajoutées à La Plata. L'importance des deux villes est évidente si on analyse les trajets des transports. Le réseau de tranways, bus et chemin de fer intégré à La Plata, était le plus dense, il n'a même pas été dépassé dans l'actualité.

L'action de l'Etat a été l'un des obstacles principaux pour un éventuel processus de mutation industrielle très important. "L'installation d'industries sur des terres fiscales n'a pas pu se concrétiser à cause de l'indécision des autorités. Ne pouvant obtenir que des permissions précaires, des entreprises comme "Ford", "General Motors", "La Cantábrica" ont renoncé à s'installer dans cet endroit." (Maselis;1982:202)

\section{6 - LA CONSOLIDATION URBAINE ET LA DIVERSIFICATION INDUSTRIELLE (1950-1975)}

Vers les années cinquante, les périodes d'excédent et de stabilité économique que traversait le pays, avec des points de repère concrets dans la micro-région -frigorifique et raffinerie- contribuent à donner lieu à une élévation du niveau de vie de la population. (FIGURE 20bp.81)

Ensenada et Berisso se consolident en tant que villes. Les transformations territoriales concernent l'amélioration de la prestation de services. L'accès à des équipements et des services se manifeste dans la construction d'écoles, réseaux d'infrastructure énergie, eau courante, égoûts, gaz- et pavés des rues. Le tissu urbain est plus dense que la moyenne métropolitaine à cause des restrictions du milieu à l'expansion.

L'autonomie municipale d'Ensenada et Berisso par rapport à La Plata en 1957 coincide avec la disparition des tramways et le déclin des frigorifiques. Dans toute la région métropolitaine prolifèrent les "frigorifiques régionaux", moins productifs que le "Swift" et l'"Armour". Celui-ci paralyse sa production vers les années soixante à cause de sa technologie obsolète qui ne lui permet pas de faire la concurrence aux frigorifiques moyens avec de modernes processus d'élaboration et traitement de viandes et sous-produits. 
La crise des deux grands frigorifiques se manifeste en contlits par rapport aux salaires, grèves durant plus de trois mois et renvois. En 1969, le "Swift" s'unit à l'"Armour"; mais le nombre d'employés est réduit à 5.000. L'entreprise fait faillite en 1970, même s'il continue sa production jusqu'à l'année 1980 avec de la viande d'autres frigorifiques.

Cette période est une lente mais persistante mutation industrielle: production d'acide sulfurique à la "Fábrica Militar de Acido Sulfúrico" (1952); des chantiers navals à "AFNE-Astilleros y Fábricas Navales del Estado" (1953), établissement dérivé du "Arsenal Río Santiago" de 1906 et du "Astillero Río Santiago" de 1936; l'industrie pétrochimique "Koppers-IPAKO" (1962); la sidérurgie "Propulsora Siderúrgica" (1969) et la "Petroquímica General Mosconi-PGM" (1970). Sauf le premier établissement situé à Berisso, tous s'installent à Ensenada. (FIGURE 22p.83) Tandis que le territoire se consolide comme espace industriel, cette période marque le déclin des productions agricoles intensives et forestières de la zone alluviale.

Fabrique Militaire d'Acide Sulfurique. A travers un Décret de 1950 la Direction Générale de "Fabricaciones Militares" prend en charge cette production dans un établissement situé dans la darse de manoeuvres du Port. Il traite 70 tonnes/jour de matières premières du nord argentin: la Province de Salta. Il s'agit de l'une des principales industries polluantes, à cause de la disposition de dépôts de soufre à ciel ouvert. Il y a eu de nombreuses tentatives de la communauté, et même du pouvoir local, pour fermer l'établissement, ou de changer les formes de traitement, mais toutes inutiles. On a observé l'augmentation du cáncer laryngo-pulmonaire dans la micro-région.

- Chantiers Navals "AFNE". Dans cette période il est le seul chantier intégral du territoire argentin et le plus grand d'Amérique Latine. Dans son terrain de 340 hectares il occupe jusqu'à 8000 employés -période 1973/1976- avec un haut degré de qualification. Son niveau de technicité lui permet employer une grande variété de processus productifs; pas seulement de bateaux, mais aussi des machines complexes. Il atteint une production de 120.000 tonnes de capacité en brut.

- Pétrochimie "IPAKO". L'entreprise "Koppers" s'installe en Argentine depuis 1956. Elle commence à produire éthylène et polyéthylène de basse densité à Ensenada à partir 1962. En 1959 le groupe argentin "Garovaglio-Zorraquin" acquiert la plupart des capitaux nord-américains originals. 
- Sidérurgie "Propulsora Siderúrgica". Il s'agit d'un établissement demi-intégré avec une chaîne de vannage de tôles en froid, avec un port privé sur la vieille Ensenada de Barragán. Il fonctionne de manière complementaire à "SOMISA", la principale sidérurgie argentine. Il couvre presque la moitié de la demande nationale de fer-blanc. Il appartient au groupe "SADE-Techint" Il occupe dans cette période entre 1600 et 2000 employés.

- Petrochimie "General Mosconi-PGM". Elle est une entreprise de l'Etat 50\% de YPF et 50\% de "Fabricaciones Militares" Elle produit depuis 1974 une grande diversité de "basiques" L'efficacité dans son fonctionnement lui permet de réaliser de grands investissements de reconversion productive après cette période.

Durant cette période on atteint le plus grand nombre d'employés industriels dans le Grand La Plata: 30.208 à 1974. Entre les trois communes de l'agglomération urbaine, se détache Ensenada avec un Produit Brut Industriel qui représente vers 1976 95\% du produit brut municipal et $41 \%$ du Produit Brut Industriel de l'agglomération.

Dans cette période on fait aussi une sous-utilisation du Port La Plata et plusieurs essais de réactivation portuaire. Depuis 1959, l'Etat octroie à YPF $70 \%$ de ses installations. Plus de $90 \%$ du mouvement de cabotage est constitué par des combustibles. L'inauguration en 1972 d'un oléoduc entre "Destilería La Plata" et "Puerto Madero" se traduit en une réduction de l'importance comme "port pétrolier" Les mouvements de sable et cailloux acquièrent une certaine signification.

Vers la fin de cette période, le nombre d'employés industriels représentait $26 \%$ de la population d'Ensenada et $10 \%$ de Berisso. Quelle est le rapport "transformations territoriales-mutations productives" ?

La consolidation des villes est le résultat de l'effet reproducteur de l'emploi industriel dans d'autres activités urbaines. Les années soixante sont des périodes de développement commercial et des services. L'incorporation de nouveaux processus productifs dans les différentes branches industrielles est assimilé positivement par la population locale. Les niveaux de qualification technique sont assez importantes, si on compare la situation de trois ou quatre décennies auparavant. Le plus grand nombre d'habitants des villes -32.000 à Ensenada et 47.000 à Berisso- réduirait l'évidence des rapports mentionnées. 


\section{7 - LES POLES PETROCHIMIQUE ET INFORMATIQUE, LA ZONE FRANCHE DU PORT LA PLATA ET LES "VILLES-DORTOIR" (1975-1992)}

Malgré les différences notables entre les trois derniers gouvernements -militaire: 1976-1983; démocratique: 1983-1989 et à partir 1989- les processus de crise économique, d'endettement extérieur et de pauvrété sociale, sont assez semblables. Pendant le régime militaire, les stratégies de contrôle social, et souvent de répression, marquent une grande différence par rapport à la démocratie. Une autre différence, concerne actuellement la plus grande importance des politiques d'ajustement, les privatisations des entreprises publiques, la stabilité économique et les projets de reconversion productive.

Dans ce cadre, l'origine du Pôle Pétrochimique le plus important de l'Argentine, à Ensenada, semble ne pas être compatible avec le processus général de rétraction du marché intérieur argentin. Plus encore, l'augmentation de niveau de chômage dans la micro-région et la transformation de Berisso en "ville-dortoir", ont lieu dans un contexte de forts mutations industrielles. Cette tendance se renforce notammenı à partir de 1989, avec la naissance du Pôle d'Informatique et de Télécommunications dans la friche industrielle du frigorifique "Swift" à Berisso. Récemment s'ajoutent de nouveaux cas de reconversion du Pôle Pétrochimique, qui représentent ensemble des investissements de plus 400 millions de dollars.

Hormis les Pôles Pétrochimique et Informatique, il existe d'autres interventions, projets et situations qui altèrent le rapport "territoireproduction" (FIGURE 22) Il s'agit de:

* La provincialisation du Port La Plata et la nouvelle Zone Franche, pas encore définie.

* La création du "Polygone Industriel", projet de refonctionnalisation de friches industrielles à Berisso.

* La paralysation de l'activité des chantiers navals "AFNE" et les conflits des travailleurs.

* L'augmentation des niveaux de pollution dans la micro-région due à de nouveaux établissements et aux reconversions productives.

* La grande rétraction des activités sylvicoles et agricoles intensives dans la zone alluviale. 
* La dégradation croissante de la plaine marécageuse, support de nouvelles activités.

* L'éventuelle réalisation du Pont International Argentine-Uruguay à partir de Punta Lara (Ensenada)

Les reconversions technologiques dans la raffinerie YPF, dans la pétrochimie PGM et dans d'autres industries, qui commencent l'automatisation de la production, les stratégies de rationalisation de la dépense publique et les politiques de flexibilité du travail; expliquent à la fois le processus de mutation productive et le chômage industriel.

Entre 1974 et 1985, le nombre d'employés du secteur secondaire se réduit notamment: de 6147 à 1780 pour Berisso; de 10539 à 6628 pour Ensenada. Cependant, le nombre de commerces et l'emploi commercial se maintient dans ces deux villes.

Les effets de cette mutation peuvent se rencontrer dans la prolifération de sous-emplois et d'emplois administratifs et commerciaux aux faibles rémunerations dans la ville de La Plata, capitale provinciale. Tandis que le nombre d'emplois commerciaux passe de 4217 à 4213 à Ensenada-Berisso pour la période 1974-1985, la variation à La Plata est de 28221 à 34169. Vers 1987 on estime qu'il y a dans l'agglomération plus de 60.000 personnes dans le secteur "informel" et 55.000 dans les administrations provinciale et municipales.

Les "transformations territoriales" définissent vers cette période une tendance à la valorisation industrielle, qui en renforçe d'autres la dévalorisation de l'environnement et la dévalorisation du support territorial comme espace résidentiel.

Le ralentissement de la croissance démographique est une manifestation de ces tendances, particulièrement à Ensenada; elle enregistre 29500, 31000, 27500 et 30300 habitants en 1960, 1970, 1980 et 1991, respectivement. Pour les mêmes années, la ville de Berisso a 40700, 44500, 50700 et 55200. (FIG URE 25)

Comment expliquer la croissance démographique de Berisso dans une cadre de dévalorisation foncière et de dégradation de l'environnement? Il répresente la possibilité d'accès au logement à bas coût pour une proportion significative de la population appauvrie, donc des logements de mauvaise qualité. Cependant, il est probable qu'il y a une autre composante culturelle: la ville est la "Capitale Provinciale de l'Immigrant" On a identifié 32 groupes de collectivités différentes, 
surtout européennes; il existe de nombreuses pratiques qui renforcen 1 les liens. Malgré la crise, ils restent à Berisso.

\section{a) Le Pôle Pétrochimique d'Ensenada}

La configuration territoriale de l'industrie pétrochimique argentine es। constituée par trois "Pôles" ou "Complexes" à Ensenada -le plus important-, à San Lorenzo, Santa Fé et à Bahía Blanca; et de nombreux petits et moyens établissements non integrés avec des technologies démodées. Il s'agit d'une centaine d'industries appartenant à quarante entreprises ayant une capacité de 2,6 millions tonnes/an de produits pétrochimiques. Ils emploient 27.000 personnes et génèrent des produits pour 900 millions de dollars/an environ. Il s'agit de la branche industrielle argentine la plus dynamique: la croissance a été de $80 \%$ durant la dernière décennie.

La pétrochimie argentine trouve son origine en 1942 dans la Raffinerie La Plata de YPF, par décision de la Diréction Générale de "Fabricaciones Militares", particulièrement de leur directeur le Général Manuel Savio. Elle naît comme un "complexe pétrochimique" Vingt ans après on inaugure la pétrochimie d'"Ipako" et vers 1974, "PGM" La période 1974-1983 est de formation et complementarité du Pôle. Les derniers investissements, entre 1988 et 1993 , se traduisent par la consolidation et la modernisation du Pôle, surtout à partir des plans de reconversion technologique.

La localisation de la Raffinerie de YPF a été déterminante dans le développement du Pôle: elle signifie la disponibilité de matière première et la possibilité de échanges de produits entre industries. (FIGURE 22)

Actuellement, il y a d'autres élements d'incitation à l'installation industrielle, pas seulement pour Ensenada. Il s'agit de la prochaine privatisation ou du changement de régime des entreprises publiques ("PGM" et "YPF"); et de la possibilité de faire des investissements productifs incluant d'avantageux mécanismes de capitalisation de la dette extérieure ("Petrokén")

Raffinerie La Plata YPF. Elle couvre une superficie de 360 ha, entre trois villes et le port. (FIGURE 22) Elle traite $50 \%$ du pétrole brut raffiné en Argentine, et représente 0,2\% du Produit Industriel Brut national. Vers 1990 elle employait 6500 techniciens et ouvriers, 55\% de l'entreprise et $45 \%$, travaillant sous contrat. La capacité de production est supérieure à $40.000 \mathrm{~m} 3$ ou $260 \mathrm{kbaril} /$ jour. 
Les principales reconversions technologiques de la raffinerie datent de 1978, 1986 et 1992. La première est constitué par le "Complejo Alquilbenceno-Cumeno" qui élabore des produits de base pour détergents, plastiques et peintures à partir du benzène de "PGM" Vers 1986 s'inaugure le "Craqueur Catalytique"; il produit des bruits très forts qui selon la direction du vent nuisent jusqu'à un demi million d'habitants.

La dernière reconversion est la plus importante par rapport à l'introduction concurrentielle dans le marché international d'hydrocarbures sans plomb. Il s'agit d'un investissement de 21 millions de dollars connue comme "Unidad de Reformado Catalítico", dont l'achévement est prévu pour décembre 1993. Les bénéfices concernent, parmi d'autres, l'augmentation de $20 \%$ de la capacité de traitement; l'élévation du nombre d'octanes de 94 à 100 et la réduction des temps d'inactivité de l'usine.

Cependant, YPF-La Plata traverse d'autres situations conflictuelles; deux sont à l'échelle locale: pollution et emploi; et l'autre, reliée à leur privatisation.

Durant 1992, en conséquence des fortes émanations gazeuses qui gênent une grande partie de l'agglomération urbaine, il $y$ a eu une situation polémique entre l'entreprise publique YPF et le pouvoir provincial, representé par le Ministère de la Santé et l'Action Sociale. Les agents polluants identifiés sont des hydrocarbures, oxyde de soufre, oxyde d'azote et plomb. YPF ne reconnaît pas en être la source d'émission. Vers la fin de 1992 on va créer un système permanent de contrôle de l'environnement, avec la participation des trois municipes concernés et la Province.

La réduction du nombre d'employés permanents, et particulièrement de "sous-traitants" a pour objectif de préparer le scénario pour la privatisation. Dans ce cadre de nombreuses entreprises moyennes d'origine locale se penchent vers l'investissement en capital de risque. Les estimations actuelles sur le nombre d'employés varient entre 3.000 et 3.500 personnes; la moitié par rapport à 10 ou 12 ans auparavant. En 1989 YPF avait au total 52.000 employés: 35.000 effectifs et 17.000 en embauche dite "contractuelle"; actuellement il s'agit de 16.000 et vers la fin 1992, elle atteindrait les 10.000 employés.

L'autre aspect conflictuel est relié à l'éventuelle privatisation de l'entreprise. En juin 1992 le Sénat National a approuvé le projet de Loi de "Fédéralisation d'Hydrocarbures" qui inclue la privatisation de "YPF" Jusqu'en Septembre 1992 il n'y avait pas d'accord à la Chambre de 
Députés. Après la vente d'actifs et d'actions on pense obtenir 8.000 millions de dollars. Selon le projet du Sénat, les gisements se transfèrent aux provinces; l'entreprise se transforme en société anonyme; $51 \%$ des actions restent à l'Etat, $39 \%$ aux provinces et $10 \%$ aux travailleurs et $100 \%$ de la vente d'actions passerá au régime de prévision sociale.

Parmi d'autres, il existe une offre d'un "holding" pétrolier composé de "Astra", "Pérez Companc", "Pluspetrol", "Tecpetrol" de "Techint" et "Bridas" Il propose d'acquérir $30 \%$ des actions.

La diréction de YPF propose une répartition finale du capital de YPF avec l'Etat national 22,6\%; les Provinces 17,4\%; le personel propre $10 \%$ et le secteur privé $50 \%$.

Pétrochimie "General Mosconi-PGM". L'établissement, ouvert vers la fin de l'autre période, continue sa croissance et la diversification de sa production de bases utilisés dans les industries éléctronique, automobile, de peinture, de refrigération et textile.

Vers décembre 1990 elle a ouvert le "Complejo Aprovechamiento de Olefinas", destiné à l'exportation et la substitution d'importations de produits de plus grande valeur ajoutée. Il s'agit d'un investissement de 180 millions de dollars. Depuis 1992 la "Unidad Oxo-Alcoholes" est en fonctionnement et produit de l'iso-octanol pour les industries pharmaceutique et de cosmétiques.

A partir de l'annonce de leur privatisation le nombre d'employés à diminué, en passant de quelque 1500 à 950 actuellement. Il y a eu une résistance à la privatisation puisqu'il s'agit de l'une des entreprises publiques les plus performantes.

On estime que vers la fin de 1992, "PGM" n'appartiendra plus à l'Etat. YPF conservera $30 \%$ de l'entreprise. Les groupes les plus interessés sont "Shell", "Pérez Companc" et des capitaux d'origine japonaise.

"Ipako". La première pétrochimie d'Ensenada est associée a celles du groupe Garovaglio-Zorraquín: "Polisur", "Petroquímica Bahía Blanca" et depuis cette année, "Petrokén" Elle produit $57 \%$ du polyetilène de haute et de basse densité de l'Argentine, pour en fournir à 1400 entreprises transformatrices appartenant à l'industrie plastique.

L'entreprise réalise un programme de récyclage de plastiques pour réduire le volume d'ordures solides. 
"Maleic". Depuis 1981 elle produit de l'anhydride maléique et couvrs le marché national avec 6 à $10 \mathrm{mil}$ tn/an. Il occupe moins de $10 c$ employés. Il s'agit de capitaux nationaux.

"Polibutenos Argentinos" et "Aco" Il s'agit de deux petits usine: qui appartiennent au groupe Bridas et fonctionnent de manièrs complémentaire avec YPF. La première produit des polybutènes et poly isobutènes pour les industries d'adhésifs et d'additifs. "Aco" fait de: alquilphénols pour les industries de cosmétiques et de lubrifiants. Il: occupent un nombre réduit d'employés.

Les quatre dernières enterprises mentionnées n'ont pas provoqué de: manifestations locales dues à la pollution. Ce n'est pas le cas de li suivante, de coke petrólier.

"Copetro". C'est la seule usine de calcination de coke résiduel d, pétrole en Argentine. Elle est formée par des capitaux nord-américains japonais et argentins ("Astra"). Elle produite depuis 1983 du coke dı haute pureté pour l'industrie de l'aluminium. Elle emploie 60 personnes (FIGURE 27)

Il s'agit d'une usine d'un haut niveau technologique. Le dernier projet en réalisation, vise au doublement de sa capacité de production; on $v$ : incorporer à l'établissement seulement 15 personnes supplémentaires.

Depuis son ouverture, le fonctionnement a provoqué un des plu grands conflits sociaux à cause de la pollution dans la micro-région particulièrement à "Barrio Campamento" Cette année il y a eu un demande judiciaire à l'enterprise; mais presque dix ans de réclamation sans réponses concrètes du pouvoir publique se sont passés. L chercheur J.Catoggio a fait un avertissement sur les risques des usine d'une telle nature: "Aux Etats-Unis il y a seulement six usines de coke responsables de l'émission de $80 \%$ des hydrocarbures aromatique polycycliques générés dans tout le pays"(El Día;16-6-91)

"Gases de Ensenada-GESA". L'établissement, aux capitaux français traite depuis 1990, des fluides industriels pour la consommatio: d'autres entreprises du Pôle: "YPF" et "PGM"

"Petrokén". Elle produit du polypropylène depuis 1992. Il s'agit d'u investissement de 180 millions de dollars d' "Ipako" (51\%) e "Shell"(49\%). Sa capacité de production est de 100.000 tn/an et 1 principale destination est l'exportation, surtout en Chine Populaire pou la fabrication de jouets. 
L'usine a été construite totalement au Japon par la "Chiyoda Corporation" avec une technologie de pointe et transportée en modules. Les travaux de remplissage de terrain dans la zone marécageuse se sont faits á des niveaux plus hauts que pour les autres pétrochimies, à cause de l'innondation de 1989, qui a précédé sa construction. Le remplissage du terrain marécageux dans le coeur du Pôle a contribué à augmenter les difficultés pré-existantes de drainage superficiel et souterrain.

La haute automatisation du processus productif est la principale cause du bas nombre d'employés, a peu près 120 .

L'un des principaux avantages de ce projet a été l'opportunité de le faire, dans une bonne proportion, sous des mécanismes de capitalisation de la dette extérieure à travers des titres.

\section{b) Le Pôle Technologique-Industriel d'Informatique et de Télécommunications de Berisso.}

Les industries informatique et de télécommunications en Argentine ne sont pas les plus développées. Par opposition à la pétrochimie, il n'y a pas encore des concentrations en Pôles ou Complexes. L'activité est assez dispersée; les installations de la région métropolitaine, particulièrement à la Capitale Fédérale, furent suivies d'autres investissements à l'intérieur liés surtout aux régimes de promotion industrielle des années soixant-dix et quatre-vingts.

L'absence de politiques orientées vers la géneration de la capacité industrielle et technologique dans le Complexe Electronique, commence à se modifier à l'époque de la démocratie. Par le Decret 621 de 1984 on crée la "CNI", Comission Nationale d'Informatique, composée de 13 organismes publiques. Elle réunit les industries d'informatique, télécommunications, éléctronique industrielle et composants.(Azpiazu et al; 1986: 220)

En 1988 le Gouvernement de la Province de Buenos Aires commence un projet de reconversion industrielle, orienté vers la récupération des installations abandonées du Frigorifique "Swift" à Berisso, sur le Port La Plata. Il couvre une superficie de $80.000 \mathrm{~m} 2$, dont $30.000 \mathrm{~m} 2$ restent couverts et disponibles pour l'installation d'entreprises. (FIGURES 22 et 26)

En 1990 s'inaugure le Pôle Informatique et de Télécommunications; et un an après le "Polygone Industriel", voisin de celui-ci. Les deux 
fonctionnent avec des systèmes de commodats de cinq ans et optior pour deux ans de plus. Le Pôle offre un bas coût de loyer: 1 dollar/mois le $\mathrm{m} 2$ et gratuit si l'entreprise recycle le bâti.

Le plus grand problème est la non-résolution légale sur la juridictior des terres. Cette incertitude juridique a été jusqu'à présent le facteuı déterminant du ralentissement des investissement à risques. En aoû 1992, après de longues démarches, la Chambre de Députés Provinciale sanctionne la loi qui déclare d'utilité publique et sujettes à expropriatior les installations du "Swift"

Le Conseil d'Administration du Pôle, "Beritec-Fondation Berissc Technologie", estime maintenant que cette décision produira un développement remarquable. Le Conseil de "Beritec" est composée du Secrétaire Général du Gouvernement Provincial, du Président de l'Université Nationale de La Plata et des membres des entreprises du Pôle. Il existe un projet d'incorporation d'un représentant de la Mairie de Berisso au Conseil.

L'un des objectifs principaux de "Beritec" est la promotion d'entreprises de l'intérieur du pays, non connectés entre elles, pouı faciliter la complémentarité et amèliorer l'efficacité du fonctionnement.

Le sous-secrétariat d'Informatique Provinciale, en relation au Programme d'Informatisation de l'administration publique, réalise depuis 1990 des cours pour son personnel. On a formé plus de 4000 employés. L'incorporation de 500 ordinateurs dans les services publics de La Plata est prévue dans un court délai.

Le même le sous-secrétariat a lancé depuis 1991 le Programme "Emprender", à travers lequel les universitaires qui présentent des projets innovateurs peuvent accéder à l'usage de l'équipement existante au Pôle. Le but est faire des micro-entreprises d'innovation technologique.

A partir 1992 fonctionne dans le Pôle, un Centre de Formation pour la population locale. Il prétend accueillir 400 éleves.

Le Pôle occupe $25 \%$ des installations du batiment reutilisé. Actuellement, il s'agit de dix-neuf entreprises, à savoir: "IBM", "Microsistemas S.A.", "Tevycom-Fapeco", "Consad", "American Security International", "Prosoft", "CPG-Sistemas Generales", "North Data", "Centro de Cómputos de Tandil", "Proceda-Grafa División Informática", "Impsat S.A.", "Codebar \& Systems", "Osdama Control", "Polo Telefónico", 
"Electromecánica Reinoso", "Make Informática", "Mapfre-Aconcagua", "SB Sistemas" et "Compumax"

Parmi les activités spécialisées du Pôle on remarque les suivantes: le premier laboratoire de dessin industriel de micro-circuits de l'Argentine; un laboratoire de calcul parallèle, nouvelle tendance destinée à faire des "PC" plus rapides; des "concentrateurs téléphoniques"; des centrales téléphoniques privées ; des réseaux de données par satellite; des micro-systèmes pour la bio-technologie; des dessins de lecteurs; et fabrication de transformateurs.

Actuellement une expérience connue comme l'"Incubatrice d'Entreprises" commence à se développer, composée d'un groupe d'universités européennes et par les universités argentines de $\mathrm{La}$ Plata, Buenos Aires et Belgrano. Le coordinateur du programme, Ing.E.Willis exprime que cette initiative se structure à partir du modèle du Silicon Valley des années soixante-dix et des expériences postériéures européenes.(El Día; 31-8-92)

Les premiers essais auront lieu dans le Pôle ainsi que dans les laboratoires universitaires qui développent les projets; dans tous les cas, il existera l'intervention des entreprises. La fonction de l'incubatrice est la génération d'unités de production et de prestation de services. Le Pôle offrira l'infrastructure et les paquets nécessaires que chaque entreprise ne peut réaliser seule. Il est prévu pour la première étape une subvention destinée à des cours de formation, des bourses et l'appui au démarrage de projets.

Il faut ajouter que récemment le Conseil des Recteurs des Universités de la Communauté Européene à travers le Programme "Columbus" a choisi l'Université Nationale de La Plata pour développer l'éxperience d'incubatrice d'entreprises.

\section{c)Le "Polygone Industriel".}

Installé dans la friche du "Swift", le Polygone Industriel de $94.000 \mathrm{~m} 2$, a traversé les mêmes vicissitudes que leur voisin, le Pôle Informatique. Des 50 entreprises actuellement en "commodat", seulement 12 produisent et exportent; 20 autres attendent leur admission. (FIGURE 22 )

La "fièvre" pour l'installation industrielle est en rapport avec les attentes autour des bénéfices éventuels du régime futur pour la Zone 
Franche du Port. Cependant, les négotiations sur la localisation de cette zone semblent indiquer un penchant vers la rive de Ensenada.

Depuis leur début, la Province, la Mairie locale, la Chambre de Commerce et Industrie de Berisso et le Copropiété d'Entreprises on accepté de faire des investissements pour 600.000 dollars en réfection, services et sécurité. Le groupe prévoit des investissements futurs pour 590 millions de dollars.

Actuellement des industries très diversifiées sont en fonctionnement; elles occupent 400 employés. A savoir: des valves industrielles; des chaises metalliques; des produits de fibre de verre; des maisons préfabriquées; des baignoires d'hydro-massages; des champignons; des membranes d'asphalte; des chaussures; de la fonderie; de pièces automobiles; et charpenteries.

Parmi les projets en cours, les plus rémarquables sont une industrie de papeterie et un autre frigorifique. La première estime faire un investissement de 15 millions de dollars, avec un système de production et de traitement de carton à travers une ligne de machines d'un haut niveau technologique. L'autre constitue un projet destiné à couvrir $12.000 \mathrm{~m} 2$ par la fabrication d'hamburguers et de traitement en froid pour ceux-ci et pour d'autres produits.

Le problème de la proprieté de la terre est autre, récemment résolu. Dans l'actuelle chambre du polygone -"CEPIB"- on estime que les difficultés de approvisionnement en énergie électrique, réduisent significativement les possibilités d'automatisation des processus productifs. Donc, pour le moment la production est surtout fondée sur l'usage de la main d'oeuvre.

\section{d) Le Port La Plata et la Zone Franche.}

Après de nombreux essais de réactivation et réfonctionnalisation, particulièrement durant la dernière décennie, où se sont inscrits des projets et des propositions très divers, comme ceux de port de conteneurs, port alternatif de Buenos Aires, régimes d'exploitation et concession avec différents buts, station de "ferry-boats" vers l'Uruguay, installation de capitaux de Coreé du Sud et Taiwán; le 8 mai 1991 on signe le Decret de Transfert du Port de la Nation à la Province.

La Plata est le septième port argentin par son mouvement annuel: 5.325.228 th en 1990 (Administración General de Puertos; 1991); constituée pour la plupart d'entrées et sorties de combustibles. Malgré 
son obsolescence, il reste le meilleur port fluvial argentin, grâce à se conditions d'accès. Le cas du Port de Buenos Aires est très sérieux: ol estime un temps de vie utile de moins de vingt ans; aprés il ne sera plu utilisable.

Les limitations pour leur réactivation ne sont pas naturelles; elles son politiques. Il s'agit d'autre cas de la forte centralisation de Buenos Aires Combien de ressources ont été destinées pendant le siècle dernier aı maintient en opération partielle du Port de Buenos Aires ? Combien dı temps Buenos Aires a eu le triste privilège d'être l'un des dix ports le plus chers du monde?

Pour le Port La Plata, l'opportunité actuelle est historique. Li réglementation récente de la vieille Loi Nationale 5142 de 1907 pou l'instalation d'une Zone Franche, pas encore définie; l'éventuel régime dı concession ou privatisation du Port par la Province; la prochain refonctionnalisation d'espaces portuaires à Buenos Aires, en aire: commerciales ou résidentielles; et d'autres manifestations de $l_{i}$ réactivation productive dans la micro-región; constituent les élements : prendre en considération pour le futur immédiat.

En 1992 l'Etat a établi un régime d'exemption d'impôts nationaux pou: les usagers qui s'installent dans la Zone Franche. Il couvre l'énergir électrique, l'eau courante, le gaz, les télécommunications et les égoûts. I existe la même démarche dans le Gouvernement Provincial.

Cependant, il reste à définir l'endroit de localisation de la Zon Franche. Maintenant la Province a un terrain de 17 ha à $17 \mathrm{~km}$ du Port L'éventuelle provincialisation des chantiers navals "AFNE" aurait pou: but, leur utilisation comme zone franche dans une aire de 170 ha.

Le destin de la Zone Franche est incertain. On considère une premièr étape commerciale, pour l'emplacement des marchandises en transit Après, le projet de l'Etat et la Province serait celui d'autorise l'installation d'industries de produits non-fabriqués jusqu'à présent er Argentine.

En ce qui concerne le Port il y a eu un projet d'exploitation intégrale avec des investissements de 60 millions de dollars. Le promoteur es "CAIP-Consejo de Iniciativas Privadas", de capitaux nationaux. En bre on propose des terminaux de conteneurs des deux côtés du Port; unt autre terminal de marchandises générales et un poste pour produits inflammables sur la rive de Berisso. 
Une ligne de "ferry-boat" qui fonctionnera à partir du début 1993 vient d'être approuvée. Elle reliera très vite Berisso avec le village de Juan Lacaze, entre Colonia et Montevideo.

Bien qu'il ne s'agisse pas de reconversions strictement productives, tous ces projets autour du Port ont une étroite relation avec les mutations industrielles actuelles. Sans doute sont-ils les élements complementaires d'un processus de mutations plus profondes dans la micro-région.

\section{e) Le chantier naval "AFNE".}

Après une période de grand développement qui va jusqu'aux années soixante-dix, le chantier marche vers sa disparition. De 8000 employés en 1973-1976, le nombre s'est réduit jusqu'à 2100 actuellement. Il existe un projet pour réduire l'effectif permanent à 800 personnes et passer la juridiction de la Nation à la Province. Après on propose des alternatives de réactivation et de co-gestion

L'établissement a une superficie totale de $340 \mathrm{ha}$, dont $120.000 \mathrm{~m} 2$ sont couverts. Il dispose d'une haute technologie pas seulement pour fabriquer des bateaux, mais de diverses machines. On estime que leur fonctionnement à plein pourrait donner du travail à 12.000 personnes. (FIGURE 22)

L'un des principales limitations en est économique. Le "Fondo de la Marina Mercante" ne donne pas les ressources pour la construction d'un "pétrolier" D'autres contrats signés n'ont pas pu se concrétiser; avec "Ferrocarriles Argentinos", "Ferrocarril del Uruguay" et les travaux du barrage "Yaciretá"

Le débat actuel se fonde sur la refonctionnalisation -d'espace productif en espace portuaire-commercial- ou sur la réactivation en profitant de l'importante infrastructure productive. Il existe aussi des intêrets pour investir des capitaux espagnols et allemands.

\section{f) Le Pont International Argentine-Uruguay.}

La réalisation d'un pont traversant le Río de la Plata entre l'Argentine et l'Uruguay est un ancien projet. Vers 1961 on croyait qu'il pourrait se construire en deux ans. 
Après des nombreuses essais, en avril 1991, la Comission Binationale signe l'Accord sur le Cadre Général que doit régir les termes de réference de l'Etude de faisabilité du Pont, en fonction de ce qui a été établi à Rome en 1988. On accepte le critère uruguayen d'octroyer plus de poids à la rentabilité financière dans le but d'attirer des investissements.

Le débat actuel se situe à nouveau sur l'interêt centraliste de faire la tête du Pont à Buenos Aires. La distance depuis la capitale nationale à la ville uruguayenne de Colonia est de plus $50 \mathrm{~km}$ et on doit faire deux grands ouvrages pour traverser les canaux de navigation. Par contre, Punta Lara, dans le Partido de Ensenada est l'endroit le plus proche de Colonia: $36,6 \mathrm{~km}$ et en plus on doit traverser un seul cannal.

Jusqu'à présent on a démontré l'interêt des capitaux divers: argentins, uruguayens, anglais, français, australiens, hollandais, nord-américains et d'autres.

\section{g) Le déclin des productions intensives.}

La zone alluviale est, malgré des innondations périodiques, une aire de haute fertilité pour les cultures maraîchères, les vignes et la forêt. (FIGURES 22 et 23)

Les descendants des immigrants, pour la plupart italiens, ont emigré dans les villes de l'agglomération. Les zones se localisent à Berisso; l'une est l'"Isla Paulino"; les autres, structurées territorialement à partir de la Route Provinciale 15 et des chemins intérieurs "La Florida" et "3 de Abril", dans la zone rurale dite "Los Talas"

Vers la période de plus grand développement on estime qu'il y avait à "Los Talas", 800 ha de cultures intensives, tandis qu'à "Isla Paulino", un peu plus de 100 ha. Maintenant, il reste moins d'un tiers de cette superficie.

Le déclin des activités intensives répond à la concurrence inégale avec l'agriculture commerciale des grandes producteurs de la région métropolitaine, particulièrement La Plata. La déterioration de l'environnement a joué un rôle déterminante dans cette crise: la pollution atmosphérique, la pollution de l'eau superficielle et souterraine, les carrières de "coquilles calcaires" à "Los Talas" et l'érosion des côtes par les vagues des bateaux à "Isla Paulino" 
h) La déterioration du milieu.

Les mutations productives et les interventions urbaines dans les dernières quinze années dans la micro-région ont lieu sur un milieu très fragile.

La somme de successifs remplissages des terrains industriels dans la plaine marécageuse a contribué à la production des difficultés de drainage superficiel et souterrain des zones intérieures plus hautes, particulièrement la ville de La Plata.

Les hauts niveaux de pollution de l'air sont produits principalement par la Raffinerie La Plata de YPF, la Fabrique Militaire d'Acide Sulfurique et Copetro. Malgré les importants parcs boisés de la microrégion et les forêts naturelles et artificielles de la zone alluviale, la dégradation du milieu s'accélère pendant la dernière décennie.

\section{Transformations territoriales et mutations productives récentes}

La micro-région d'Ensenada-Berisso traverse actuellement d'importantes transformations territoriales dues à des mutations productives. Des processus parallèles de valorisation et dévalorisation de diverses logiques y interviennent.

Dans la mesure où l'industrie se consolide à travers les dernières reconversions productives, la fragilité du milieu naturel ne parvient pas à répondre d'une manière efficace. Le territoire se transforme en un espace portuaire-industriel. La vocation vraiment urbaine se perd et les productions agricoles intensives tendent à disparaître.

Un milieu ayant ces caractéristiques ne peut pas être le récepteur de toutes les logiques qui régissent les transformations territoriales pendant son histoire. Les mutations industrielles sont l'un des principaux déterminants de cette limitation structurelle.

Dans les conclusions de cette recherche on donne des précisions sur le rapport "transformations territoriales-mutations productives" dans la micro-région; pour finir avec un essai de généralisation conceptuelle et méthodologique après l'analyse des mêmes rapports dans la région métropolitaine et dans l'autre micro-région. 
FIGURE 19

LA MICRO-REGION DE ENSENADA ET BERISSO

TRANSFORMATIONS TERRITORIALES ET MUTATIONS PRODUCTIVES

a) LES "SUERTES DE ESTANCIA"

c) LES "SALADEROS"

b) LE "FUERTE DE LA ENSENADA"

d) LE PORT ET LES FRIGORIFIQUES
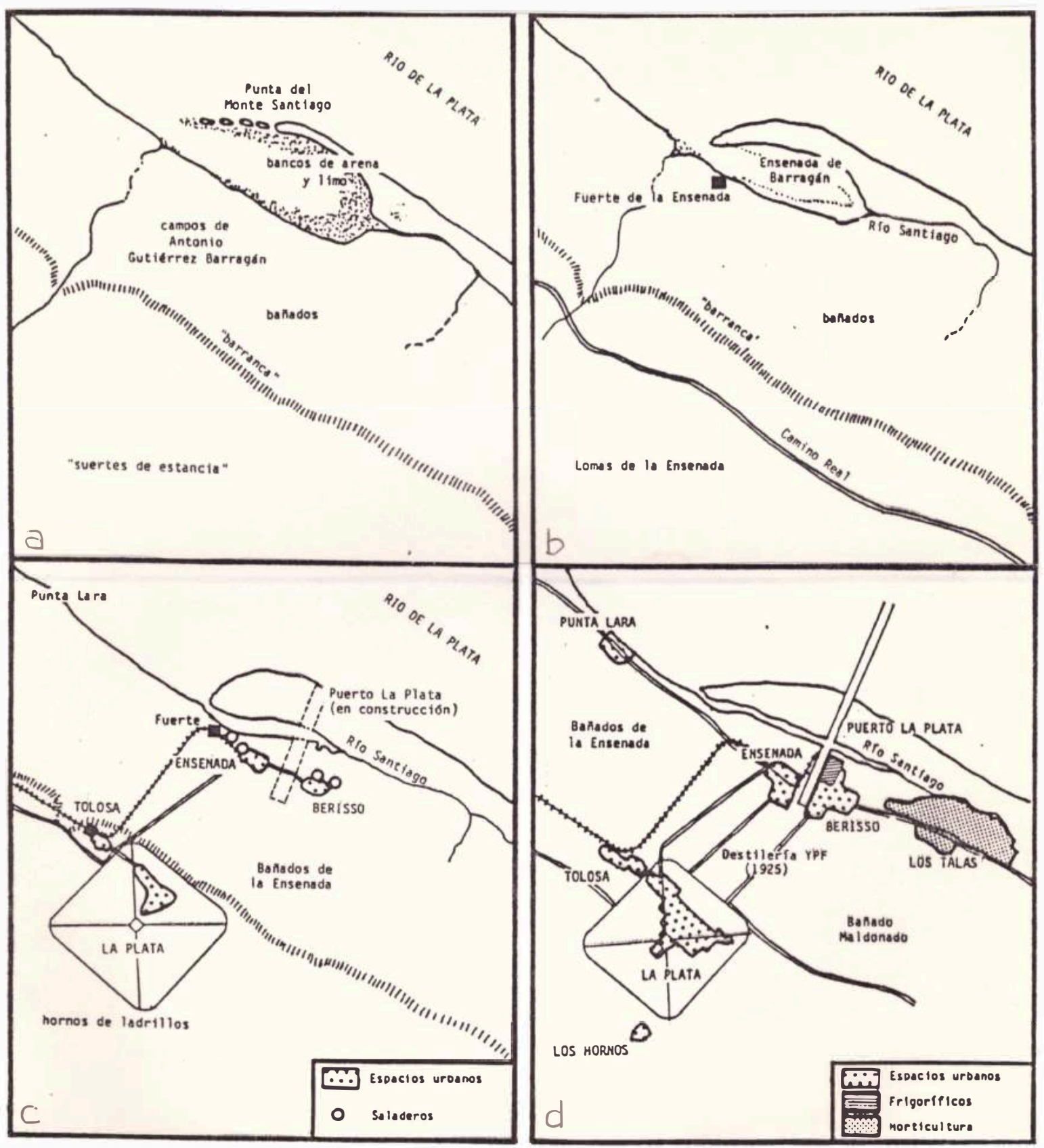

Source: Laurelli, E. et Bozzano, H. (1991) In: Revista Interamericana de Planificación. Elaboration personnelle. 
FIGURE 20

\section{LA MICRO-REGION DE ENSENADA ET BERISSO}

TRANSFORMATIONS TERRITORIALES ET MUTATIONS PRODUCTIVES

a) LA RAFFINERIE DE YPF

b) LA DIVERSIFICATION INDUSTRIELLE

c) LES POLES PETROCHIMIQUE ET INFORMATIQUE d) PROFIL DU RELIEF
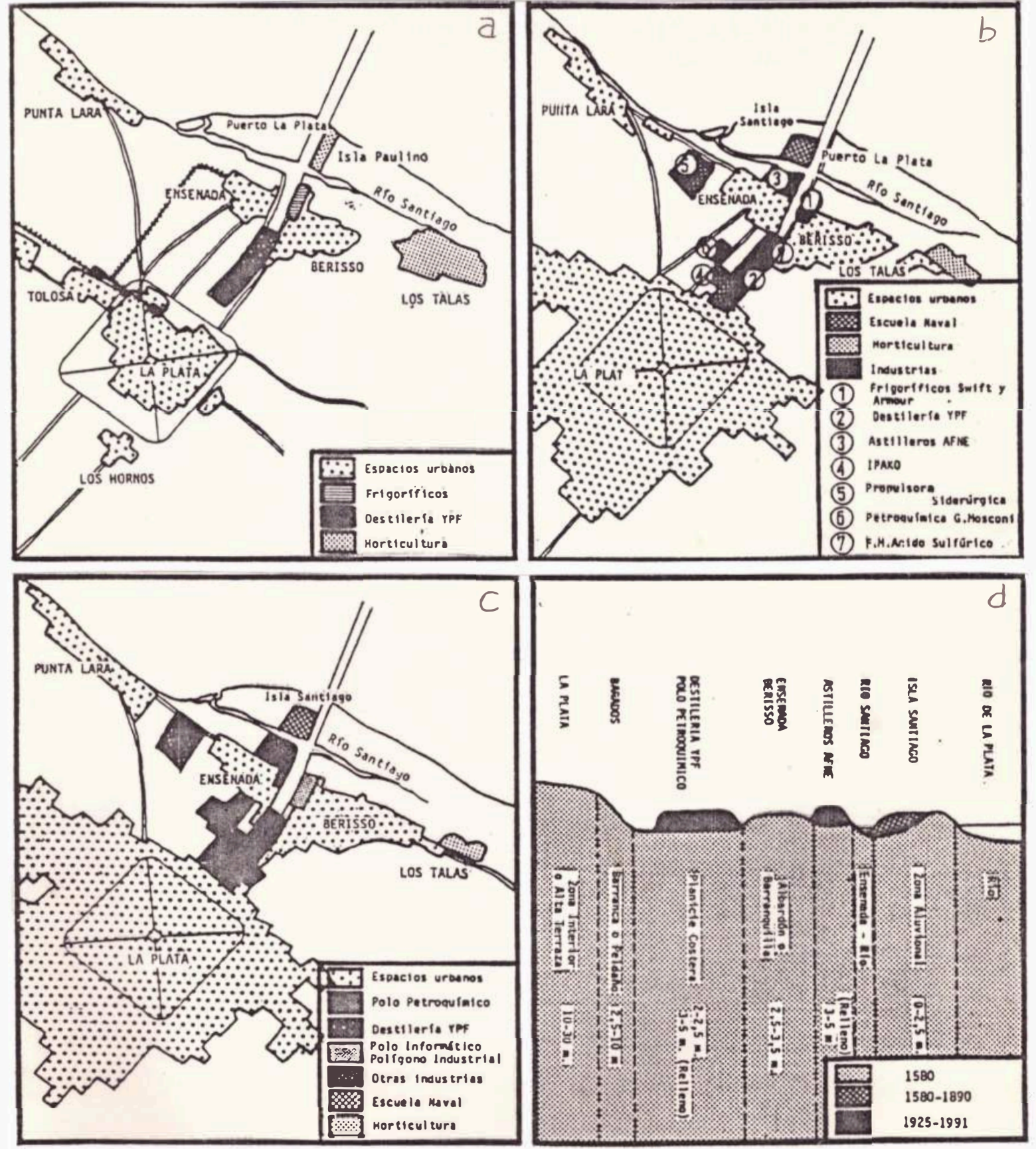

Source: Laurelli et Bozzano (1991) op.cit. Elaboration personnelle 
FIGURE 21

RELIEF DANS LA MICRO-REGION DE ENSENADA-BERISSO

Les courbes de niveau de $2,5 \mathrm{~m}$ et de $5 \mathrm{~m}$ sont différenciées par deux nuances de vert. Ils incluent la "Plaine Cotière" du Río de la Plata. On peut identifier la petite ravine dit "albardón" où se situent les villes d'Ensenada et Berisso.

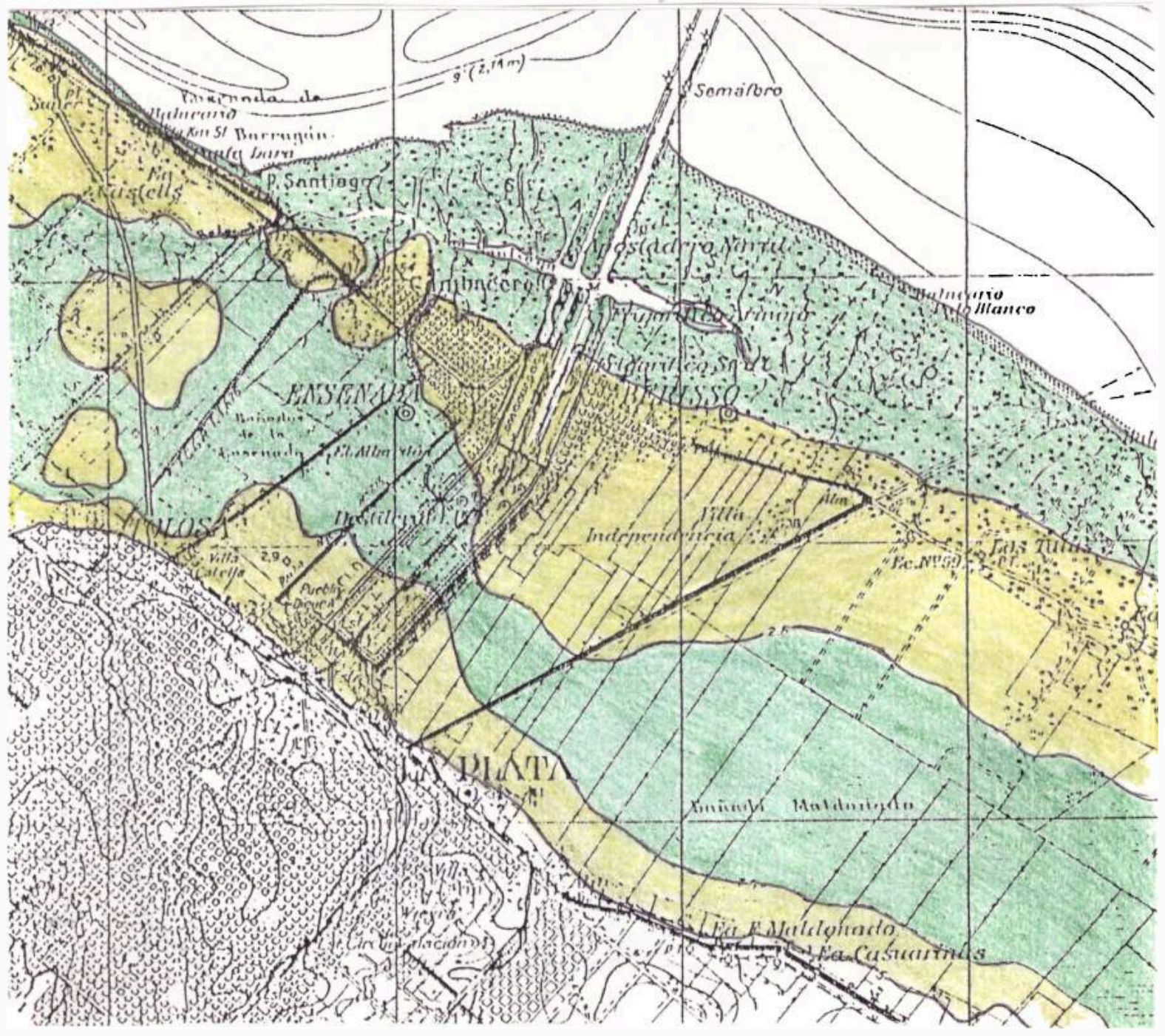

Source: Carte Topographique "La Plala" Militaire Argentin.

Echelle 1:100.000. Institut Géographique 
FIGURE 22

\section{L'USAGE DU SOL DANS LA MICRO-REGION DE ENSENADA- BERISSO}

(ELABORATION PERSONNELLE A PARTIR DE PHOTOGRAPHIE AERIENNE DE 1984)

ESPACE URBAIN RESIDENTIFL

PLUS 50\% ESPACE OCCUPE

20/50\% ESPACE OCCUPE EQUIPEMENTS

MOINS 20\% ESPACE OCCUPE

ESPACES VACANTS OU NON-UTIL.ISES

ILOTS

PARCELLES RURALES

FRICHE INDUSTRIELLE

EX-HORTICULTURE
ESPACES PRODUCTIFS

INDUSTRIES

INFRASTRUCTURES ET

ANCIENNE CARRIERE

CHORTICULTURE

BOIS

एक्यात्य ELEVAGE

ESPACE DE RECREATION

PARC/VILLAS

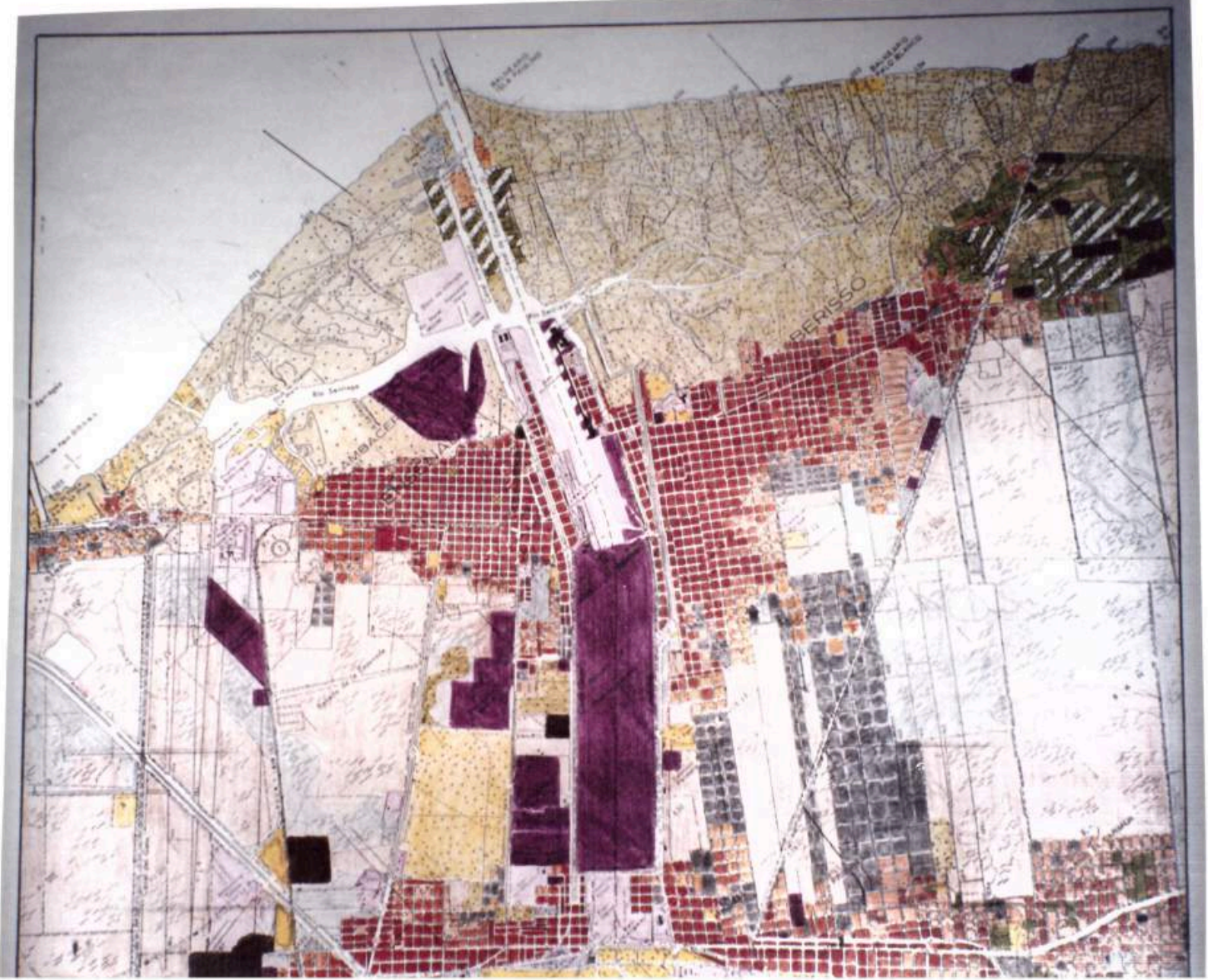


FIGURE 23

\section{LA MICRO-REGION DE ENSENADA ET BERISSO LES PRODUCTIONS INDUSTRIELLE ET AGRICOLE-INTENSIVE}

ON IDENTIFIE UNE AIRE INDUSTRIELLE AUX ENVIRONS DU PORT, EN DETACHANT LA RAFFINERIE DE YPF. LES DEUX AIRES MARAICHERES SONT EN RECUL (ALTERNANCE DE HACHURES BLANCHES ET VERTES)

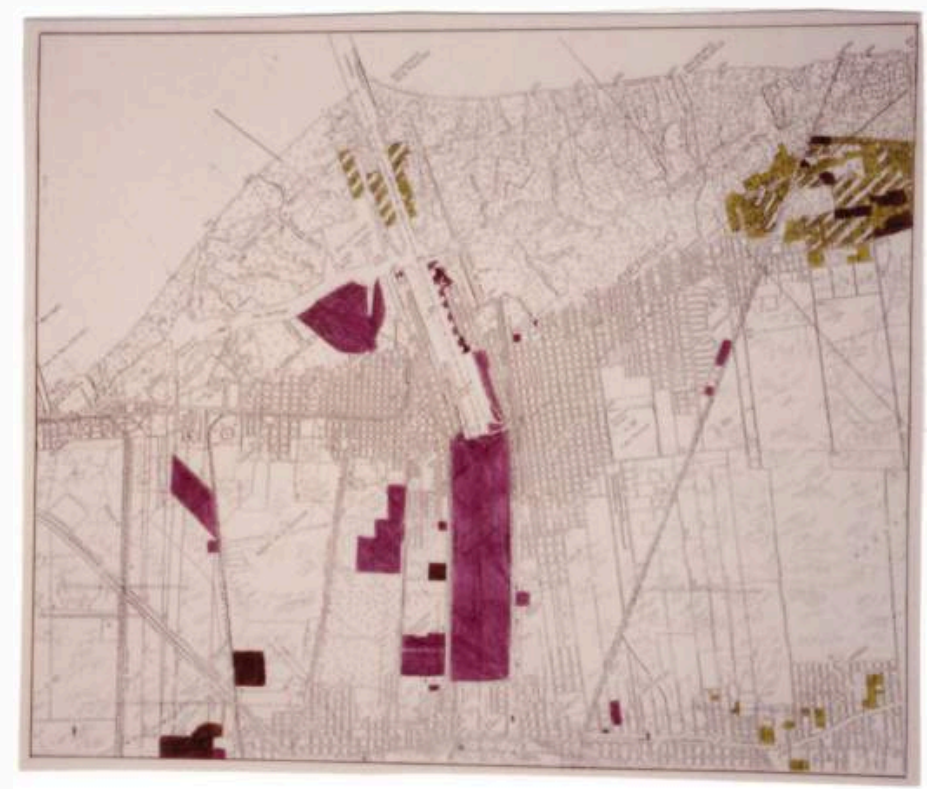

FIGURE 24

LA MICRO-REGION DE ENSENADA ET BERISSO LES ESPACES PRODUCTIFS

LA PLAINE MARECAGEUSE NE CONSTITUE PAS UN SUPPORT NATUREL APTE A LA PRATIQUE D'ACTIVITES ECONOMIQUES. ON PEUT IDENTIFIER LA FAIBLE OCCUPATION POUR L'ELEVAGE.

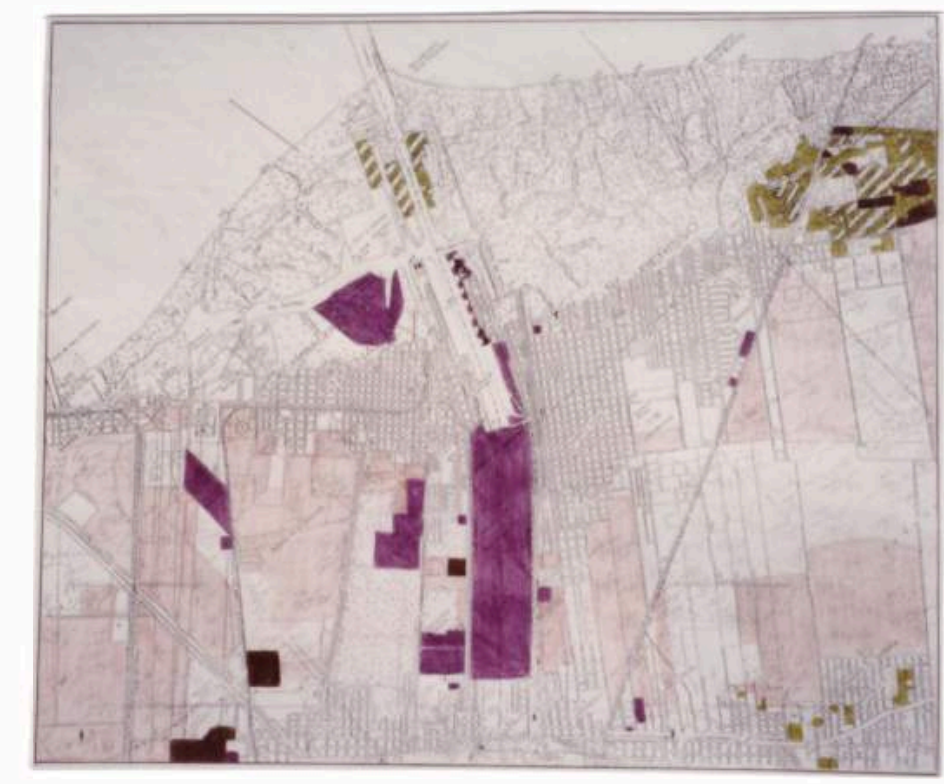

Elaboration personnelle à partir de photographies aériennes de 1984. 
FIGURE 25

LA MICRO-REGION DE ENSENADA ET BERISSO

EVOLUTION DE LA SUBDIVISION DE LA TERRE

LQTISSEMENTS URBAINS

ANTERIEURES A 1915

$1915 / 1947$

$1947 / 1965$

$1965 / 1992$
PETITES SUBDIVISIONS RURALES

(PARCELLES INFERIEURES DE 5 HA)

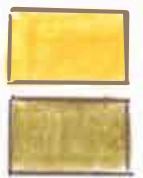

$1947 / 1965$

$1965 / 1992$

LA COURBE DE NIVEAU DE 2,5 M INDIQUE LES DIFFERENCES DOUCES D'ALTITUDE ENTRE LA PLAINE MARECAGEUSE ("BAÑADO") ET LA PETITE RAVINE ("ALBARDON").

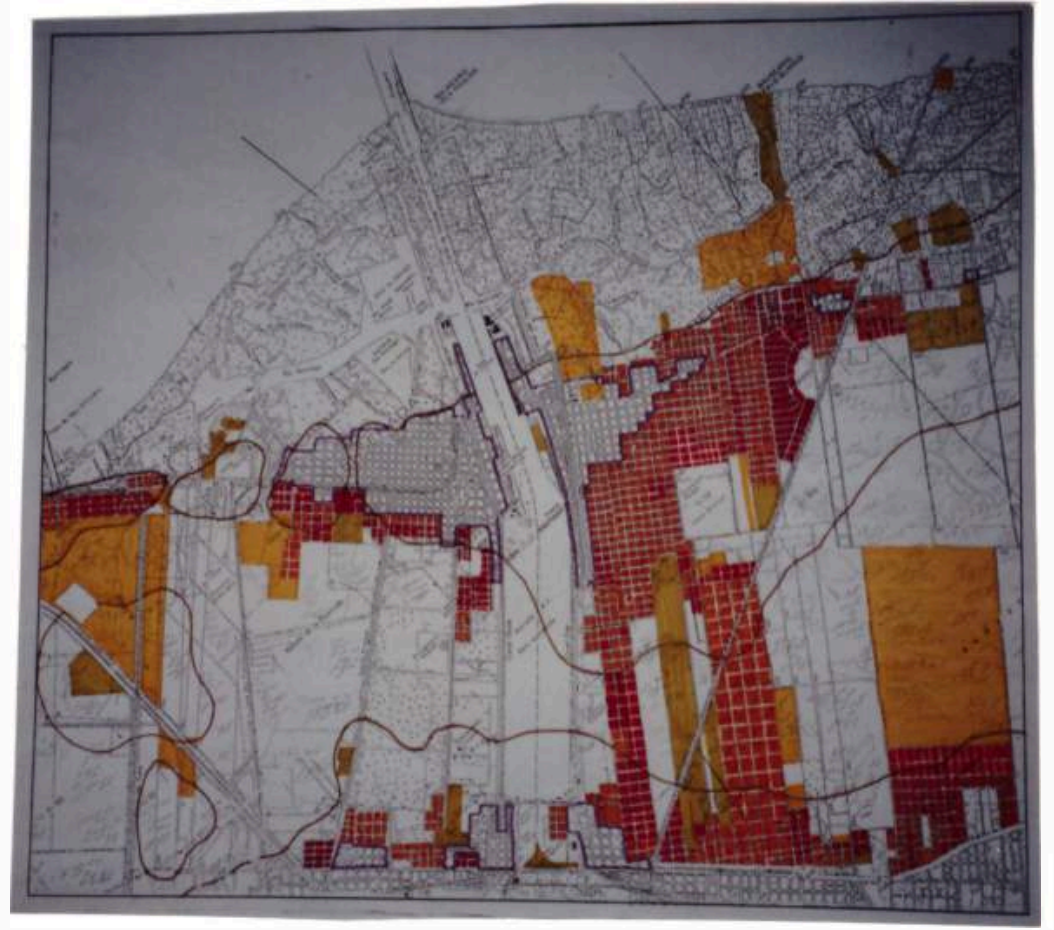

Source: Peirano,G., A.González et H.Bozzano. "Evolución de la subdivisión de la tierra en La Plata, Ensenada y Berisso" (inédit) 
FIGURE 26

\section{LE POLE INFORMATIQUE ET DE TELECOMMUNICATIONS DE BERISSO}

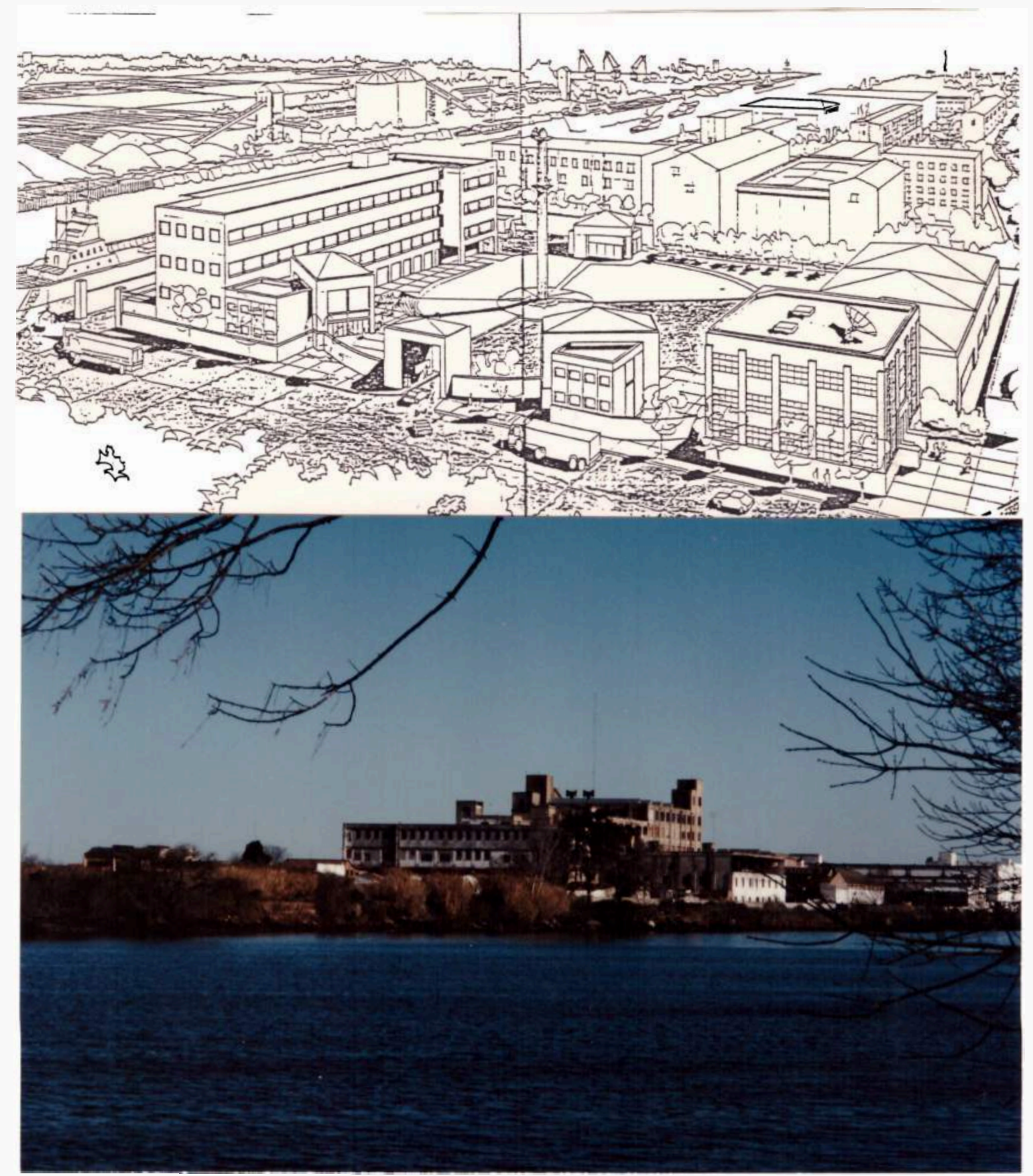

Sources: Dessin: Provincia de Buenos Aires (1990) "El Polo Tecnológico-Industrial de Berisso" Photo: H.Bozzano-1991. 
FIGURE 27

\section{LE POLE PETROCHIMIQUE DE ENSENADA}

LA PETROCHIMIE "GENERAL MOSCONI-PGM"

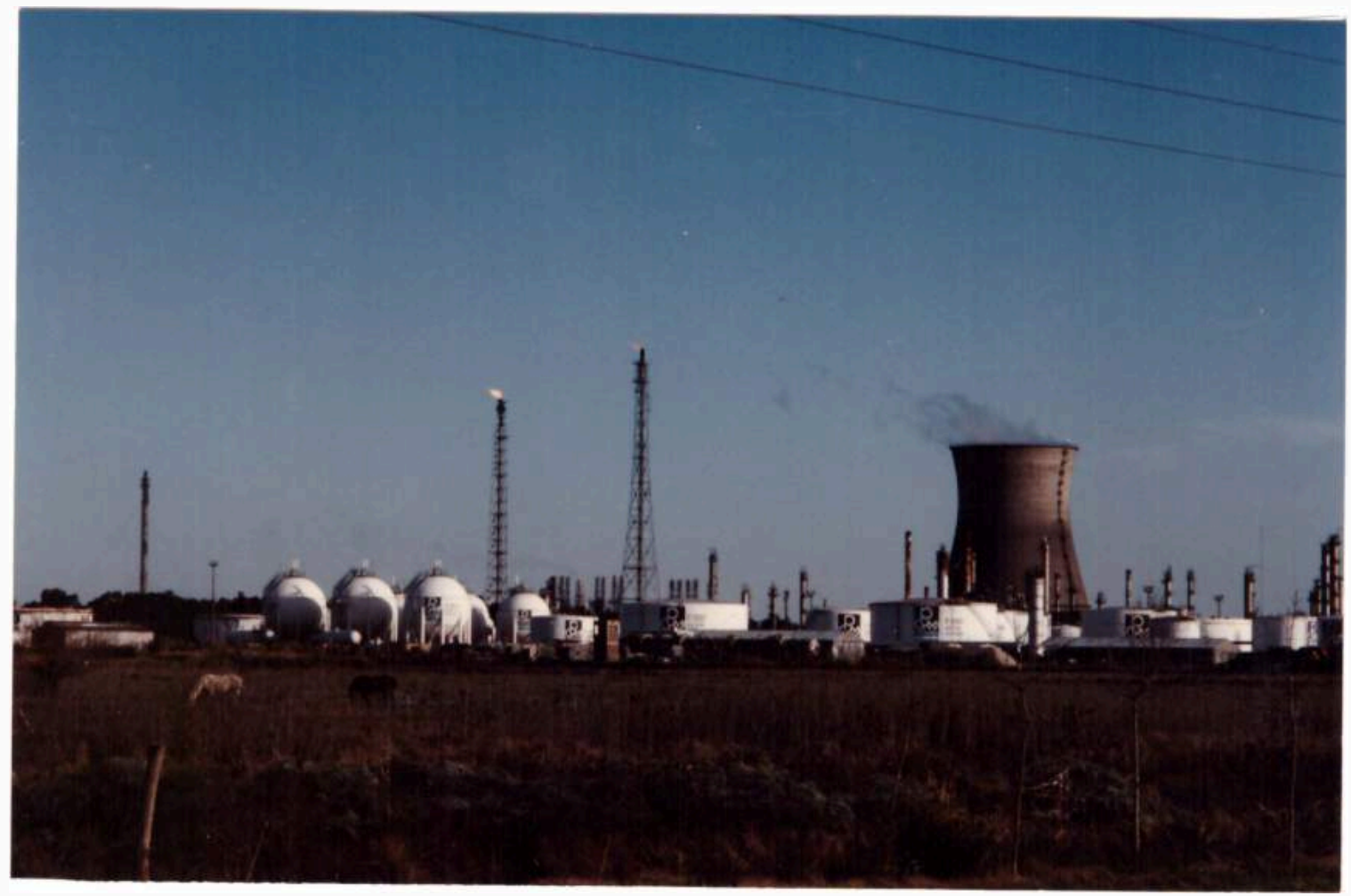

L'USINE "COPETRO" DE COKE RESIDUEL

LA STATION FERROVIAIRE INACTIVE DE "RIO SANTIAGO"

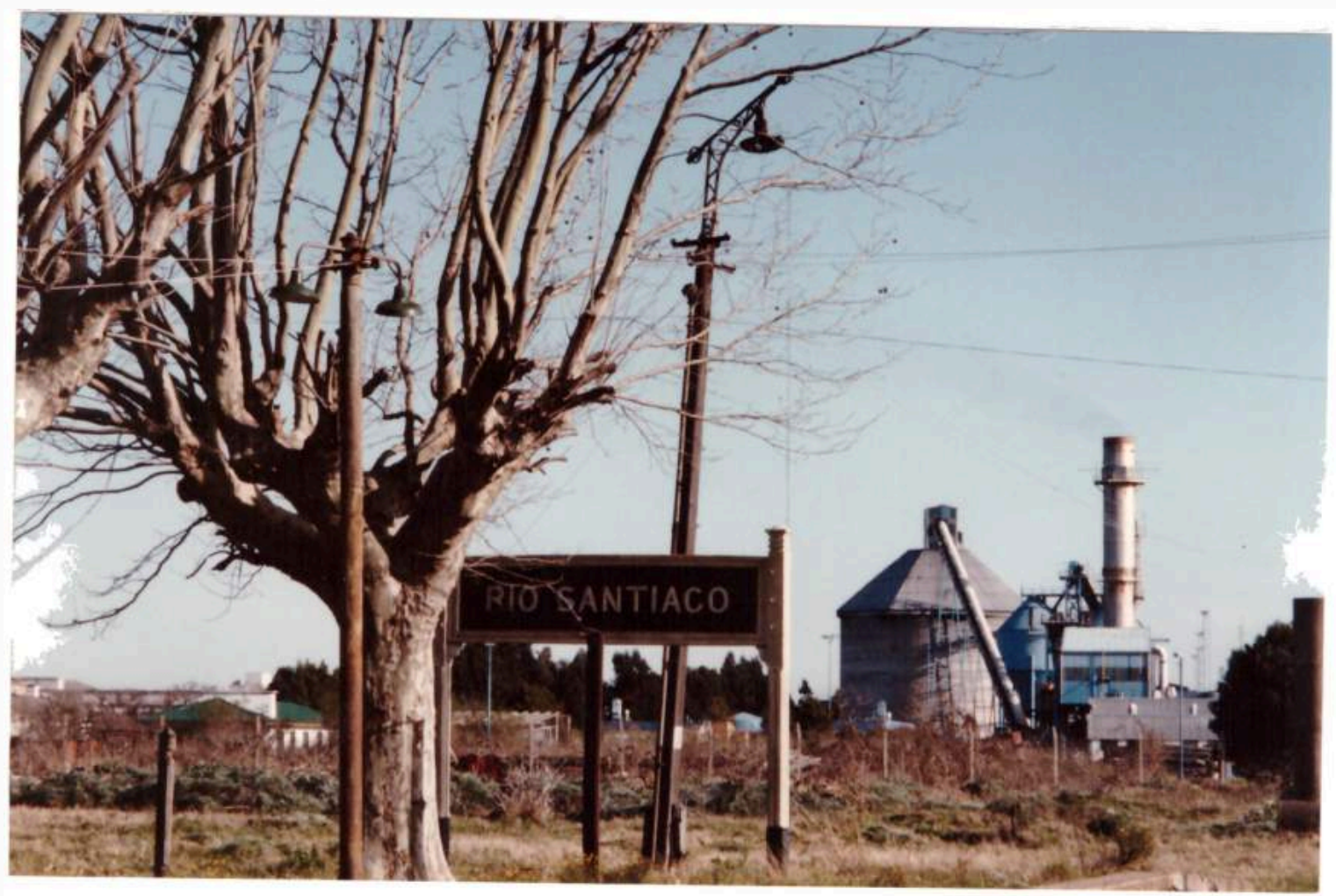




\section{4 - LA MICRO-REGION DE \\ ING. ALLAN-EL PATO-EL PELIGRO}

La micro-région choisie (64 km2 et 23.800 habitants) est l'un des extrêmes de la Région Métropolitaine vers le Sud-est, occupant des portions des municipes de Florencio Varela (Ingeniero Allan), Berazategui (El Pato) et La Plata (El Peligro). (FIGURES 17 et 18)

Le territoire présente les caractéristiques communes de tous ceux que l'on nomme "tentacules métropolitains" à Buenos Aires, sauf dans le cas de l'inexistence préalable de villages. Les transformations territoriales et les mutations productives sont récentes. La structure agraire de la "pampa" s'est maintenue jusqu'en 1950. L'activité maraîchère, les lottisements et l'industrialisation se succèdent et se juxtaposent rapidement pendant moins de trois décennies.

Actuellement le territoire traverse les vicissitudes d'un espace très périphérique en crise et reconversion. Il est situé à une heure de Buenos Aires en voiture, temps qui se multiplie par deux ou quatre selon le moyen de transport et la distance du logement aux couloirs urbains. L'impact d'une grande industrie -"Peugeot"- à été déterminant durant la brève histoire de la micro-région.

Les mutations industrielles et agricoles intensives de la micro-région sont répresentatives de processus socio-économiques plus généraux. Il est possible de faire une périodisation en isolant les manifestations locales des situations globales.

1 La mise en valeur des "pampas" à partir d'élevage destiné à l'exportation et leur continuité pendant la durée du modèle "agroexportateur" (1800-1945): Les "estancias" et les "tambos".

2 L'attraction de Buenos Aires dans le Cône-Sud américain et le début de la métropolisation (1945-1960): L'expansion péri-urbaine explosive.

3 La dernière industrialisation substitutive et la croissance du faubourg dit "suburbio" (1960-1975): L'impact de "Peugeot" et l'occupation des lotissements. 
4 La crise économique et sociale et la récente réconversion productive (1975-1992): La désindustrialisation, le sous-emploi et la mutation productive actuelle.

\section{Le milieu naturel.}

La micro-région constitue une portion du secteur méridionel de la "Pampa Ondulada" Cette sous-région naturelle est une frange de terrain entre les vallées du Paraná-Plata vers le nord-est et du SamborombónSalado vers le sud-ouest. Les petits fleuves qui la traversent ont déterminé les douces ondulations du terrain. (FIGURES 18 et 28)

A l'exception de la zone nord de la micro-région qui descend vers la "Planicie Costera" du Rio de la Plata, c'est le plus souvent l'aire la plus haute, entre les vallées des fleuves mentionnées et celles des ruisseaux Conchitas et Pereyra. 68\% de la micro-région a entre 25 et $30 \mathrm{~m}$ au dessus du niveau de la mer; $18 \%$ entre 20 et $25 \mathrm{~m}$; et $14 \%$ correspondent à des zones déprimées: les vallées des ruisseaux mentionnées.

Cette une zone très favorable à l'agriculture sans arrosage; le climat est tempéré humide, le sol est "brunizem planosol" et le milieu est herbacé. Les vallées ne sont pas aptes à l'agriculture, avec des sols "solonetz-alcalin" (Cappanini; 1966) Il s'agit donc, d'un territoire avec un site naturel non limitatif pour le développement d'activités. (FIGURE 28-p. 113 )

\section{TRANSFORMATIONS TERRITORIALES}

ET MUTATIONS PRODUCTIVES. UN ESSAI DE PERIODISATION.

\section{LES "ESTANCIAS" ET LES "TAMBOS" (1800-1945)}

L'aire étudiée est, parmi toutes les autres entre Buenos Aires et La Plata, la dernière à recevoir les transformations territoriales produites lors des périodes de substitution d'importations.

La micro-région est sous-utilisée jusqu'à début de ce siècle: les ovins dans les grands établissements dits "Estancias", où selon J.Lynch, une personne pouvait s'occuper de 3000 têtes de bétail. Le raffinement des moutons, connu comme la "Merinización de la Pampa", vers la décennie de 1840, introduit des changements graduels dans l'activité. Le nombre 
d'ovins dans la Province augmente notamment: 2 millions en 1810, 15 millions en 1852 et 40 millions en 1865.(Lynch; 1982:203)

L'introduction du chemin de fer vers la décennie de 1870 par des britaniques est liée à l'appropriation de terres sur la "pampa"

Vers le début de ce siècle le territoire entre Buenos Aires et La Plata présente principalement cinq modes d'occupation:

a) Localités et stations créées à partir de l'arrivée du chemin de fer durant le dernier tiers du siècle passé.

b) Nouveaux lotissements de très bas niveau d'occupation, qui couvrent lentement les interstices entre les stations les plus proches de Buenos Aires (Avellaneda et Lanús) et de manière discontinue les zones au delà de Quilmes.

c) Localisation d'industries liées à l'élevage: frigorifiques et tanneries à Avellaneda-Lanús; et à l'expansion du marché de consommation nationale: verrerie "Rigolleau" à Berazategui, brasserie "Quilmes" à Quilmes et "Maltería Hudson" à G.E.Hudson.

d) Propriétés rurales petites et moyennes destinées à la production agricole intensive et à la production laitière dites "Tambos" Les premières exploitations plus proches de Buenos Aires et La Plata; les autres, parmi d'autres, dans la micro-région.

e) Anciennes "Estancias" de grande extension avec élevage. On remarque celles des familles Pereyra Iraola, Bell et Campbell Davidson.

Malgré le passage du chemin de fer par la micro-région, seulement les établissements d'élevage s'y développent: la "Estancia Santo Domingo" de la famille Campbell Davidson, avec 5000 ha à peu près et des établissements moyens -entre 50 et 300 ha- pour la plupart "Tambos" Il s'agit particulièrement de familles créoles et d'origine basque; on peut citer les suivantes: Barragán, Sánchez, De la Fuente, Arrogui, Gorostiaga, Islas, Semper, Arrascaete, Sagastizábal et Risso.

Vers l'extrême nord de la micro-région, la station férroviaire "Juan María Gutiérrez" et une petite école rurale depuis 1916, sont les seules lieux de rencontre de la réduite population résidente.

Les antécedents liés à la transformation territoriale sont derivés du pavage de la Route Nationale 2 en 1934 et du fonctionnement du Chemin de Fer Provinciel en 1942 avec des stations dans la micro- 
région: "Ingeniero Juan Allan" et "Centro Agrícola El Pato" Les deux axes traversent l'aire concernée dans des directions opposées.

Entre 1945 et 1949, les premiers lotissements discontinus ont liet dans la micro-région aux environs de la Route Nationale 2 par groupes de 4 à 10 ilôts. En moins de 10 ans les "tambos" disparaîtront. Il se produit alors une restructuration économique et sociale profonde dans le territoire.

Il n'est pas encore possible de définir les transformations territoriales par rapport aux mutations productives. Celles-ci son très faibles.

\section{L'EXPANSION PERIURBAINE EXPLOSIVE (1945-1960)}

Durant cette période l'Argentine connait le scénario d'un développement industriel assez répandu. Il est lié à l'installation d'entreprises nationales productrices de moyens de consommation de masse, principalement dans les branches alimentaire et textile. Parallèlement, se consolident certaines entreprises transnationales installées pendant la période de la première substitution d'importations.

La réalisation massive de lotissements très périphériques est un reflet du processus d'industrialisation dans la première couronne. Il y a un décalage spatial entre la transformation territoriale de la deuxième couronne et la mutation industrielle de la première couronne. Durant la décennie de 1950 certaines industries s'installent au nord de la micro-région, entre J.M.Gutiérrez et Bosques.

Les expectatives créées dans des zones expulsives de population de l'intérieur du pays, de la Bolivie, du Paraguay et de l'Uruguay, ont lieu autour de l'image de Buenos Aires comme la "grande ville" Il y a une relation avec la croissante demande de salariés pour des industries de main d'oeuvre intensive de bas niveau technologique. C'est le début d'une tendance de tertiarisation en commerces et services divers.

Quels sont les déterminants qui permettent d'expliquer les transformations territoriales durant cette période ? Les mutations productives n'interviennent pas à l'échelle locale. En opposition, il y a une série d'aspects qui contribuent à définir la "grande affaire foncière", à savoir: l'appropriation d'une partie des subventions sociales données à travers les services de transports publics urbains; la permission de ceux qui appliquaient les normes publiques de régulation du marché foncier; 
Les lotissements populaires et les lotissements de résidences secondaires des années cinquante se trouvaient à plus de $20 \mathrm{~km} \mathrm{du}$ dernier "suburbio" à moitié consolidé. Aujourd'hui il s'agit des terres dépeuplées, non-utilisées, inserées dans une structure agraire traditionnelle. (FIGURE 32-p. 116)

Les composantes de cette transformation territoriale à la microrégion sont: la prolifération de grands lotissements urbains sans services sur les axes routier et ferroviaire; l'installation de producteurs agricoles d'origine européenne; la réalisation de fractionnements ruraux de petite taille pour la production intensive et pour la résidence-secondaire; et la radication des premières industries.

En 1950 s'installent vers le nord de la micro-région -station ferroviaire J.M.Gutiérrez- la grande industrie textile "Fábrica Argentina de Alpargatas", et "Parafina del Plata", un établissement chimique moyen. Le cas de "Alpargatas" constitue un échantillon du processus de décentralisation industrielle par densification et spécialisation résidentielle au centre-ville. Les îlots qu'elle a utilisés à partir de 1885 , passent à une proprieté de 76 ha entourée d'un milieu rural, à $34 \mathrm{~km}$ de Buenos Aires

\section{Les lotissements populaires.}

Durant une courte période $-1953 / 1965$ - on lotit $84 \%$ de la terre urbaine de la micro-région: 881 îlots sur un total de 1047. Les nouveaux lots sans services ont une capacité pour loger une population de 90.000 habitants, dans 22321 parcelles; cependant, on estime en 1960 pas plus de 3.000 habitants. (FIGURE 32-p.116)

Cette période est celle d'un développement plus important de l'activité immobilière de la région métropolitaine. Elle se caractérise par la diversification de fonctions des sociétés et groupes économiques: des capitaux appliqués à l'industrie, la production agropastorale et forestière et aux activités bancaires et financières.

Trois sociétés ont fractionné $76 \%$ des parcelles urbaines de la microrégion: 19126 sur un total de 25021. Il s'agit de "Montaraz SAICI" (Sociedad Anónima Industrial, Comercial e Inmobiliaria); "Santo Domingo SFICA" (Sociedad Financiera e Inmobiliaria en Comandita por Acciones) 
et "Guillermo Decker SAICAFI" (Sociedad Anónima Industrial Comercial Agropecuaria Financiera e Inmobiliaria). (FIGURE 32-p.116)

Les autres fractionnements ont été réalisés par 26 agents, pour la plupart des propriétaires individuels ou groupés, apparemment sans la répresentation ou l'intermédiaire d'agences immobilières ou d'autres types de sociétés. Souvent, il s'agit de subsidiaires des grandes enterprises.

Les conditions de vie durant la décennie de 1950 reflètent des situations critiques: localités sans aucun type de service dans des aires totalement rurales. Les premiers services de transport collectif -"El Cóndor" et "Río de la Plata"- s'habilitent entre 1958 et 1961 avec une très basse fréquence à travers le seul axe important: la Route Nationale 2. Certains usagers doivent marcher à pied parfois jusqu'à quinze "cuadras", soit 1500 à $1800 \mathrm{~m}$, entre leur logement et l'arrèt du transport.

Le premier service est l'énergie domiciliaire. Pour l'eau courante, les premiers groupes ont dû attendre plus de trois décennies. 40 ou 45 années après il $\mathrm{n}$ y a actuellement aucun réseau d'égoûts dans la microrégion.

\section{Les mutations agricoles.}

L'arrivée d'Italiens, d'Espagnols et de Portugais durant cette période signifie une mutation assez forte en ce qui concerne les activités agricoles. Celle-ci a été accompagnée d'un processus de fractionnement rural qui dépasse l'intervention des mêmes agriculteurs, en incluant des sociétés de capitaux diversifiés.

Entre 1945 et 1960 se fractionnent dans la micro-région 818 parcelles de moins de 5 ha de superficie moyenne. Plus de la moitié -435appartiennent à un lotissement de la même entreprise qui constitue le plus grand lotissement urbain dans le territoire étudié: "Montaraz" Il est situé dans le Partido de Florencio Varela, le moins développé en ce qui concerne les productions intensives. (FIGURE 32)

Les étrangers réalisent la mutation agricole, tandis que les sociétés locales pratiquent la même spéculation foncière que dans les lotissements urbains. 
Les liens familiaux et amicaux à l'intérieur des collectivités d'étrangers sont déterminants pour l'expansion des activités productives. Beaucoup travaillaient aux exploitations laitières ("tambos"), agricoles ("chacras") et d'élevage ("estancias"), mais la plupart d'entre eux ont lieu à travers la diffusion personnelle des relations sociales. (Tadeo; 1991:15)

Cette période signifie la disparition de la "estancia", la déclination du "tambo", la prolifération de la "quinta" maraîchère et le début de la floriculture.

Les premières zones exploitées se trouvent aux environs des Routes Nationale 2 et Provinciale 36: les vieilles "Estancias El Peligro" et "Peligro Chico" à La Plata, et "Estancias Lavalle" et "Santa Rosa" à Berazategui. (FIGURE 29)

Bien que la mutation productive ait été importante en ce qui concerne le passage des activités agricoles extensives à des activités intensives, cette intensité est rendue plus par l'incorporation de main d'oeuvre que par des capitaux dans les secteurs des technologies et agro-chimiques. Cependant, les transformations territoriales majeures au bénéfice de mutations agricoles sont propres de la période postérieure.

\section{3 - L'IMPACT DE "PEUGEOT", L'OCCUPATION DES LOTISSEMENTS ET L'EXPANSION DES PRODUCTIONS INTENSIVES (1960-1975)}

Entre 1958 et 1974, sauf certains va-et-vient, l'Argentine traverse la période de développement le plus intense depuis la decennie de 1920. La micro-région constitue un exemple excellent, par les transformations territoriales, ainsi que par les mutations productives qui s'y déploient.

Cette période se caractérise par: a) l'investissement de capitaux productifs étrangers destinés principalement à couvrir la demande intérieure; b) la subordination des petites et moyennes enterprises nationales aux dits capitaux, en termes de financement et de pouvoir de détermination de prix du marché intérieur; c) l'ampliation en nombre des secteurs des revenus moyens, et moyen-bas en moindre mesure. associés à cette industrialisation substitutive; et d) le développemenı d'un Etat producteur de biens et de services pour différents secteurs sociaux (Azpiazu et al; 1986) 
A cela s'ajoute la discontinuité du système démocratique qui intervient comme limitation à un développement social majeur. Tandis que les gouvernements militaires privilègient des politiques de distribution régressive des revenus; les gouvernements démocratiques mettent l'accent sur la rédistribution. Avec, facteur aggravant, des rapides interruptions de politiques sociales en 1966 et 1976: les "coups militaires" des Présidents A.H.Illia et M.E.M.de Perón.

Les "politiques d'assignation et de production" (O'Connor, en Wright et al;1986) privilégient les investissements en infrastructures sociales ou économiques, selon leur orientation dominante respectivement à l'amélioration directe des conditions de vie ou à l'appui aux activités économiques. La crise à partir de 1975 réduit notamment tous les niveaux d'investissement; situation qui continuerait jusqu'à présent.

Le développement économique et social généralisé se manifeste dans la micro-région par: a) l'installation d'une grande usine automotrice de capitaux français: "Peugeot"; b) l'acquisition de lots et l'incorporation de nouveaux habitants à la métropole; et c) la croissance soutenue de l'horticulture et de la floriculture.

\section{a) L'impact de "Peugeot"}

La période 1958-1962 est celle du plus grand investissement productif industriel en Argentine. Des 597 millions de dollars investis, $90 \%$ correspondent à des capitaux étrangers, orientés vers les secteurs chimique, petrochimique et de l'automotrice. (Lindenboim; 1987:16)

L'expansion industrielle des grandes entreprises durant cette période -comme "Peugeot"- répond davantage à l'augmentation de la demande intérieure qui a des franchises, des exemptions et d'autres bénéfices des régimes de promotion industrielle.

Sûrement aucun habitant de la micro-région n'ignore la signification de "Peugeot" pour leur territoire tout le long de $15 \mathrm{~km}$ par la Route Nationale 2.

Les parcelles urbaines sont rapidement occupées l'influence de "Peugeot" est plus indirecte que directe. La main d'oeuvre de niveau de qualification moyen et haut provenait d'autres zones de la région métropolitaine; seulement entre 5 et $10 \%$ venaient des localités el quartiers voisins, généralement avec des bas niveaux de qualification: nettoyage et autres services. 
L'influence indirecte par rapport aux activités connexes développées: commerciales, de services, ou industrielles en moindre mesure, de fournisseurs de l'enterprise, ainsi que la multiplication d'activités donnée par cette distribution relative de revenus à l'échelle locale. La même période signifie l'augmentation de la fréquence du service de transport public, circonstance qui contribue à l'amélioration des conditions d'accès des habitants dans le sud de la région.

La "Sociedad Automóviles Peugeot" s'installe en Argentine vers 1959 à Buenos Aires. La même année on déboise une parcelle de "El Pato", Berazategui, sur la Route Nationale 2, à $42 \mathrm{~km}$ de Buenos Aires. Le développement de l'enterprise est soutenu pendant deux décennies, malgré les conflits politiques et économiques qui ont traversé l'Argentine dans la même période. La production automotrice croît significativement, ainsi que les revenus des employés et la qualité des couvertures sociales. (FIGURES 29 et 30)

La production se multiplie par trois en quinze ans, en passant de 48 automobiles par jour en 1962, à 95 en 1966 et 160 entre 1976 et 1978 . Le salaire moyen d'un employé de "Peugeot" était à peu près le double de celui d'un employé de l'autre grande usine de la micro-région: "Alpargatas"

La productivité de la filiale argentine en relation à la production globale de l'entreprise, mesurée en termes comparatifs "automobiles/employé" est baisse. Vers 1970 la Société avait 48.000 employés qui produisaient 2.500 automobiles/jour; tandis que dans l'usine argentine les 4600 employés produisaient la moitié de la moyenne. La population consultée relève comme cause de cette situation, l'utilisation d'une technologie productive un peu plus ancienne et des coûts relativement bas de la main d'oeuvre durant cette période.

Mais, il ne s'agit pas d'un développement linéaire, soutenu et harmonique à "Peugeot" d'Argentine; vers 1963, l'Administration Nationale de Douanes vérifie une commission de contrebande qualifiée et réitérée par l'entreprise argentine que dirigeait l'industrie: "IAFAIndustriales Argentinos Fabricantes de Automotores" sous licence "Peugeot"; l'instruction sommaire est soumise à la Justice Fédérale; après un litige de onze années, on condamne "IAFA" à payer des amendes supérieures à la valeur totale de l'usine.

A ce conflit, s'ajoutent les mécanismes de mise en marche des accords de travail, directement entre l'entreprise et le syndicat national SMATA, et certaines relations avec la clientèle dit "clientelares" et des "amitiés 
internes" Tous ces facteurs réduisent l'efficacité du fonctionnement de l'usine.

\section{b) L'occupation des lotissements.}

Quels sont les facteurs déterminants des transformations territoriales après deux decennies de dépeuplement et de lotissements vacants dans la micro-région?

Le peuplement du territoire est constitué principalement par des groupes d'immigrants à bas revenus et capacité d'épargne réduite. Les déterminants de cette situation sont: a)l'installation des usines "Alpargatas" et "Peugeot"; b) le "coût relatif" bas du transport public au delà de la deuxième couronne; c) la haute occupation, et souvent la saturation, des lotissements les plus proches de Buenos Aires: la première couronne; d) les difficultés d'accès à la propriété dans les quartiers suburbains les plus consolidés: sous-centres de la deuxième couronne; et e) l'offre soutenue d'emplois urbains pour toute la région.

Vers la fin des années soixante des petits centres commerciaux sont créés avec quelques équipements collectifs à "El Pato" et "La Carolina"; un peu plus tard à "San Pablo" et durant le début des années soixantedix à "El Nuevo-Km 44,5" et "Barrio El Parque" (FIGURES 29 et 32)

Le processus de croissance de ces localités réproduit ceux de la consolidation des quartiers ou des localités suburbaines de la Région Métropolitaine de Buenos Aires. Sur une rue avec des commerces et quelques services -école, unité sanitaire, poste- se réalise le premier pavage; suit l'introduction d'une ligne de basse fréquence de transport collectif depuis la route la plus proche. Les voisins accèdent lentement aux services. Le réseau d'energie domiciliaire, est généralement suivi de celui d'eau, obtenu souvent de puits, l'éclairage public réduit aux coins, l'amélioration de quelques rues, les drains pour les égouts pluviaux dans les zones déprimées. Postérieurement, et pas toujours avec succès, les réseaux des égouts et de gaz naturel.

Le processus de suburbanisation enregistre différents rythmes en fonction des diverses politiques d'assignation des ressources. Vers la moitié des années soixante il y a un déclin des politiques d'assistance, dit "asistencialistas" La localisation périphérique, dans un milieu rural durant cette époque, est le facteur qui exprime le manque de projets d'infrastructure de cette nature dans la micro-région. 
Dans ce cadre, de nombreuses familles de l'intérieur du pays et des pays voisins acquièrent des lots péri-urbains, en reproduisant le même schèma suivi par les immigrants européens, quatre ou cinq décennies avant la première couronne suburbaine. Entre ces deux courants migratoires il y a des différences de contexte politique et économique, et un peu plus de capacité d'épargne et d'investissement du premier courant migratoire par rapport à la seconde.

Le processus devient plus complexe à cause des migrations intraurbaines, où se détachent des situations assez diverses: la nonoccupation des logements à louer, dits "inquilinatos" et "conventillos" au centre-ville ; l'éradication des familles des quartiers très précaires, dits "villas-miseria" dans la Capitale Fédérale. Il y a une lente transformation territoriale métropolitaine: la diminution de la densité dans certaines zones centrales et l'occupation de la périphérie urbaine.

La décennie de 1970 constitue le début d'une nouvelle tendance: la différentiation territoriale de la micro-région. Le lotissement de "Ingeniero Allan" en est le plus grande: 397 îlots avec 11289 parcelles sans services pour des secteurs populaires; il inclut trois quartiers: "La Carolina", "Barrio Parque" et "Barrio Once" Les localités de "El Pato", "San Pablo" et "Barrio Raelwi" ont des lots un peu plus vastes que ceux de "Ingeniero Allan" Le mode d'acquisition des parcelles est un autre élément de différentiation. Au delà, vers la Route Nationale 2, "El Nuevo" est un petit hameau avec quelques commerces pour la zone rurale. (FIGURES 29 et 32)

Les mutations productives interviennent-elles à la manière de facteurs déterminants des transformations territoriales? Le développement des productions intensives et l'existence préalable du "Centro Agrícola El Pato" sont en rapport avec la nature du village: un centre de services ruraux et après, la formation d'une coopérative prospère. Dans le cas de "Ingeniero Allan", par rapport à "Peugeot", bien que la plupart de ses habitants ne travaillent pas à l'usine, l'effel multiplicateur des activités est évidente.

Les habitants de "Ingeniero Allan" affirment que vers 1960 les résidents à "La Carolina" et "Barrio Parque" ne dépassaient pas 80 , et $2 \mathrm{C}$ familles. Vers la moitié des années soixante-dix on estime une population entre 6 et 8 mille habitants. Le troisième quartier -"Once"enregistre vers 1975 un indice d'occupation très bas.

Le processus d'occupation des lots urbains suit la "vague d'expansion" de la Région Métropolitaine donnée par l'amélioration des conditions d'accès. En résumé la vague en est la suivante: "J.M.Gutiérrez" (km 34 
depuis Buenos Aires) à 1960; "Ingeniero Allan" (km 37) et "El Pato" (km 39) entre 1960 et 1970; "San Pablo" et "Raelwi" (km 40) à 1965; "El Nuevo" (km 44,5) à 1968 et "ruta Sol" (km 49) à 1975. (FIGURE 32)

\section{c) L'expansion des productions intensives.}

L'occupation des terres fractionnées deux décennies auparavant, par des agriculteurs ètrangers de la micro-région répresente la consolidation d'une mutation productive initiée avec très peu de succès durant les années cinquante.

Quels sont les facteurs déterminants des transformations territoriales dans les aires agricoles ? Les aspects sont les suivants: a) une situation de démande soutenue de la consommation intérieure; b) la mise en oeuvre de "politiques redistributives"; et c) la prolifération des liens culturels entre des collectivités.

Dans les exploitations c'est le travail familial et le sous-emplois de courte durée, généralement pour des nouveaux migrants du nord argentin, qui font des tâches de collecte et de maintenance avec l'aide de leurs familles; tous travaillent, même les petits enfants. On assiste ainsi vers la decennie de 1970 à la réproduction d'une situation d'exploitation laborieuse condamnable aux portes de la métropole.

Parallèlement, vers la fin des années soixante se produit l'arrivée de nombreuses familles d'origine japonaise qui s'installent dans des zones rurales diverses à La Plata: "El Peligro", parmi d'autres. Souvent, ces Japonais avaient eu de courtes expériences de travail agricole au Brésil, au Paraguay, en Bolivie et en République Dominicaine. (Tadeo; 1991:17) (FIGURES 29 et 30)

Pendant cette période se consolide un territoire autour des productions intensives. Premièrement l'horticulture, avec les Portugais et les Italiens; à peu près, le début de la diversification des Portugais: la diffusion de la floriculture comme activité alternative. Finalement les Japonais, en floriculture. Les années 1972-1975 trouvent un aire maraîchère en pleine expansion. De même, ces années marquent la diffusion de l'activité avicole.

L'impossibilité à avoir une information ponctuelle sur le degré de technicité des activités durant cette période, limite la portée de cette analyse. Cependant, en fonction de l'information donnée à la station du "INTA-Florencio Varela" pour le sud de la région métropolitaine, il est 
sûr que l'incorporation de nouvelles technologies de production est très faible ou inexistente

Vers 1975 le développement de ces activités est telle qu'on ouvre l'ur des sièges de la Coopérative Argentine de Floriculteurs. En ce quj concerne l'horticulture, la totalité des trois municipes de la micro-régior apportent entre $40 \%$ et $50 \%$ du total des légumes produits dans la régior métropolitaine.

Le rapport "transformation territoriale-mutation productive" est très étroit. L'expansion des espaces maraîchers contribue notammen à la consolidation d'un territoire en tant que tel. La conformation d'espaces productifs plus vastes, homogènes et compacts que des exploitations isolées, intervient comme mode de frein à l'expansior urbaine.

\section{4 - LA DESINDUSTRIALISATION, LE SOUS-EMPLOI ET LA MUTATION PRODUCTIVE RECENTE (1975-1992)}

Si les années 1975-1977 sont celles de la finalisation d'une longut période de croissance de l'industrie argentine, les années 1990-1992 sont elles, celles du début d'une tendance à la reconversion productive dans plusieurs branches des activités industrielles et agricoles.

Le contexte national est caracterisé par une réduction notable de la demande intérieure et par une grande diminution des investissements La manifestation la plus forte de cette crise est le choc économique de 1975 durant la Présidence de M.E.M.de Perón.

L'irruption du gouvernement militaire en 1976 répresente ur changement de la structure socio-économique nationale. A part les tendances de rétraction économique mentionnées, on le verifie $\grave{c}$ d'autres situations d'accentuation de la crise: a) la libéralisation des marchés, en particulier le financier; b) l'égalité de droits des entreprises nationales et étrangères; c) l'action de "discipliner" la force de travail, $\grave{c}$ travers la répression et l'annulation des lois de travail; d) la désindustrialisation généralisée; et e) la diversification des capitaux étrangers.

L'avènement du gouvernement démocratique du Président R.Alfonsír en 1983 réprésente la récuperation de formes de participation des groupes sociaux à travers des demandes et des revendications 
sectorielles, et peu à peu dans des conquêtes laborales perdues pendant le régime militaire.

Le changement du gouvernement démocratique du Président C.Menem à 1989 signifie l'accentuation des politiques d'ajustement commencées deux ou trois ans auparavant. Mais aussi c'est une période de privatisations des entreprises publiques, de centralisation du capital, de réconversion productive, de stabilité économique et de pertes des niveaux en investissements sociaux.

La micro-région constitue un échantillon excellent de ces processus, à cause de la diversité et la profondeur des situations rencontrées. La désindustrialisation et la reconversion productive, d'une part; le sous-emploi et la différentiation sociale, d'autre part; voilà les manifestations les plus saillantes des actuelles transformations territoriales.

Le scénario enregistre ces manifestations concrètes: a)La décadence de "Peugeot" et la reconversion industrielle; b)Le sous-emploi et l'appauvrissement des quartiers populaires; c) La différenciation territoriale à travers la spécialisation comme une aire de résidences secondaires et loisir; et d) La crise maraîchère et floricole et le début d'une reconversion et d'une diversification des productions intensives.

\section{a) La décadence de "Peugeot".}

La désindustrialisation dans la micro-région et dans l'industrie automotrice nationale n'échappe pas au processus de désindustrialisation le plus grand de l'histoire argentine.

Les onze mille emplois industriels de la micro-région des années 1974-1977 se réduisent à trois mille pendant les années 1981-1983.

L'usine "Peugeot" à El Pato, qui a atteint un nombre d'employés supérieur à 6000, commence à décliner son activité. En 1976, durant la gestion du Ministre Martínez de Hoz se forment huit comissions pour résoudre des différends, qui en réalité étaient des sentences confirmées par la Cour de Justice: l'une de ces commissions s'occupe du "Cas Peugeot" Peu de temps après, la subsidiaire française "Safrar S.A." arrive à un accord avec la Cour: en fonction de l'intérêt social se réduisent notamment les amendes.

En 1980 surgit "Sevel Argentina S.A. à partir de la fusion de "Fiat" e1 "Safrar-Peugeot", avec une participation actionnaire de Fiat (15)\% et du 
groupe économique de Francisco Macri (85\%). L'opération consiste en des contrats à travers lesquels on transfère la plupart des actions par des "royalties" et des utilités durant des périodes données (Minsburg;1987:87). En moins de deux ans l'usine est désactivée et dégrossie. Les 4500 employés de 1980 disparaissent en 1982. Seulement $20 \%$ du personnel -le plus hiérarchisé- se transfère à l'usine "Sevel" de "El Palomar", $20 \mathrm{~km}$ environ à l'ouest du centre-ville de Buenos Aires.

Les raisons de la fermeture de l'usine de "El Pato" sont diverses; particulièrement il s'agit de la récession généralisée et de l'orientation économique globale des capitaux productifs à la circulation financière; dans une moindre mesure, s'ajoute l'utilisation de technologies et dessins souvent désuets, en rélation à d'autres filiales automotrices en Argentine.

\section{b) La reconversion de l'industrie automobile.}

Après huit ans d'inactivité, l'usine "Peugeot" est l'objet d'une refonctionnalisation par le même groupe économique qui acquiert la firme automotrice: "Sevel Argentina"

Bien qu'il n'ait pas été possible de le confirmer, on a identifié des sources diverses mais coincidentes: des tentatives d'installation entre 1988 et 1989 des grandes entreprises "Toyota", "General Motors" et l'une des deux plus grandes usines laitières argentines. Il n'a pas été possible non plus d'identifier le type de relation entretenue par chaque entreprise intéressée avec le groupe propriétaire de l'usine.

L'entreprise "CIR2-Centro Industrial Ruta 2" a pour but de profiter de l'infrastructure non-utilisée de l'usine "Peugeot" pour recevoir des industries de technologie de pointe. Elle occupe une superficie de 34 ha dont presque la moitié sont couvertes: $150.000 \mathrm{~m} 2$. Elle comprend quatre grands établissements avec tous les services où des industries peuvent s'installer. Vers 1991 fonctionnaient trois petites industries de pièces automobiles avec 78 employés et une puissance installée de 260 HP: "Strapur Argentina", "Desplast Argentina" et "Unión de Constructores de Máquinas S.A. Jusqu'en 1992 s'étaient installées quatre autres petites industries. (FIGURES 33 et 34)

Actuellement, la réactivation de l'industrie automobile argentine est remarquable. La production annuelle passe de 98.000 en 1990, à 140.000 en 1991; les estimations pour 1992 sont de 290.000 unités. Les stratégies des grandes entreprises démontrent l'intérêt par les 
investissements en Argentine, malgré les hauts coûts de production par rapport à d'autres pays.

"Cinasa-Compañía Interamericana de Automóviles" (ex-Renault) développe un plan orienté vers l'introduction en Argentine des mêmes modèles qui apparaissent à l'Europe; ainsi que l'évaluation des projets technologiques et en la modification des structures en fonction de critères ecologistes.

13 ans après sa disparition de l'Argentine, l'entreprise "General Motors" retourne, associée à "Iveco" (70\% du groupe Garfunkel et $30 \%$ de Fiat). Elle commencera à produire à Ferreyra, Córdoba, des voitures el des camions à partir de Juin 1993. Sa stratégie est de se conduire de manière complémentaire avec la filiale "General Motors" du Brésil, en profitant du cadre du "Mercosur"

En 1992, les entreprises "Ford-Autolatina" de General Pacheco (Tigre), et "Mercedes Benz" de González Catán (La Matanza), ont annoncé de grands investissements de l'ordre de 200 millions de dollars.

"Sevel Argentina", le résultat de l'union "Fiat-Peugeot", ouvrira prochainement l'usine de "El Pato" à partir d'un projet de reconversion technologique très important. On prévoit une production de 100 unités par jour, et vers 1993, entre 150 et 160. Les modèles seront: Fiat Spazio, Fiat Fiorino, Fiat Ducato et Peugeot J5. La raison en est de maintenir le premier poste du marché national de ventes, actuellement 43\%. Malgré la reconversion productive récente, l'usine "Sevel" de "El Palomar" ne parvient pas à couvrir la demande.

L'utilisation de technologie de pointe à l'usine de "El Pato" signifie une amélioration de l'efficacité du processus productif, mais aussi l'automatisation et la robotisation, donc la réduction du personnel. Tandis que pendant les années soixante-dix, 6000 personnes produisaient 160 unités par jour, on aura seulement besoin après la reconversion de quelques 500 personnes pour produire entre 100 el 120 voitures par jour. Il faut ajouter qu'en fonction de la tendance actuelle à la division en cycles productifs mineurs, d'autres établissements feront certaines parties des unités. Donc, la corrélation d $\epsilon$ productivité n'est pas précise entre ces deux périodes.

Qu'est-ce que la mutation industrielle à "Sevel" ?

A la robotisation de 1985 et 1986, succède une autre récente et plus profonde. En 1991, l'introduction d'"Iles Robotisées Flexibles" permet de faire la construction de carrosseries au même niveau des standards 
industriels internationaux en vigueur. Ces îles robotisées permettent de produire des changements de modèles plus facilement et de supprimer les "stocks inter-opérationels" Cette situation promeut le développement industriel des entreprises nationales sous contrat.

Le système flexible aéreo automatique de distribution de carrosseries couvre une aire de $3700 \mathrm{~m} 2$ à "El Palomar" et fonctionne avec des "Robocarriers" qui choisissent leur propre circuit selon le modèle d'automobile.("Clarín", 27-6-91)

A cause de l'ouverture de l'usine à "El Pato", l'entreprise a sollicité récemment de jouir des bénéfices de la Loi Provinciale 10547 de Promotion Industrielle. Des fonctionnaires estiment que ceci aura un résultat favorable: l'exemption totale des impôts de bénéfice brut et immobilier.

La nouvelle de l'ouverture a occasionné des attentes notables dans la micro-région. La liste des aspirants est de 800 personnes. Il faut estimer les niveaux de qualification réels par rapport à la reconversion technologique à mettre en oeuvre.

Les mutations industrielles et les transformations territoriales à l'échelle locale semblent indiquer un décalage croissant, pas encore évalué.

\section{c) Les autres industries dans la micro-région.}

Bien que l'impact de la fermeture de "Peugeot" soit important dans la micro-région, le processus de récession des autres usines n'est pas moins significatif. Les unes réduisent leur production; les autres, finalement ferment. (FIGURES 29 et 30)

La "Fábrica Argentina de Alpargatas" -avec $250.000 \mathrm{~m} 2$ couverts dans toute l'Argentine- réduit de $40 \%$ son personnel de l'usine locale et passe de 3000 à 1800 employés. Elle produit des biens de consommation, particulièrement des tennis et des tissus. La diversification des activités -financière, bancaire, textile, agricole, pêcheuse- lui a permis d'opérer avec une certaine flexibilité. Les quatre aspects saillants de sa rationnalité comme entreprise sont: la réduction du volume de production par contraction du marché; la reconversion technologique des cycles productifs; la diminution du nombre d'employés; et la réorientation des capitaux à d'autres activités plus rentables. 
Parmi les autres industries, la plupart occupe une main d'oeuvre qualifiée non-résidente dans la micro-région. Il s'agit de: trois pharmaceutiques ("Produfarma-Upjohn", "Abbott" et "LederleCyanamid", les deux dernières au delà de la micro-région, vers le nord); trois métallurgiques ou derivées ("Acrow", "Galaxia" et "Industrias Varela"); une fabrique de glace ("Rolito"); un abattoir et frigorifique ("La Huella") et une dizaine de petites entreprises de production variée. L'étude de ces industries sera l'objet d'un traitement plus profond dans de futures étapes du projet de recherche.

Les variations du secteur industriel durant la période 1974-1983 en Argentine, indiquent que, tandis que le nombre d'ouvriers diminue de $34 \%$, les heures/ouvrier, de $30 \%$ et le salaire réel, de $17 \%$, la relation productivité/coût salariel révèle une appropriation importante des excédents par le secteur entrepreneur.(Azpiazu; 1986:103)

La chûte de l'investissement productif et la "financiérisation" de l'économie donnent lieu à une grande rétraction et reconversion du marché intérieur une tendance est le passage de la production de produits de consommation de masse à celle de produits somptueux pendant la période 1975-1990. La désindustrialisation en Argentine es1 critique. Entre 1980 et 1982, le produit brut industriel diminue presque de 25\% (Azpiazu; 1986:106)

d) Le sous-emploi et l'appauvrissement des quartiers populaires.

L'autre face de la récession montre une marginalisation croissante d'un vaste secteur de la société depuis 1975, en ce qui concerne le marché du travail et les conditions de vie. Tandis que quelques groupes augmentent leurs excédents, la base sociale s'appauvrit et s'agrandit. Les manifestations de ce processus dans la micro-région sont évidentes.

L'industrialisation et la suburbanisation ne maintiennent pas, depuis les années soixant-dix, le rythme qu'elles ont eu pendant les années soixante.

Le début de la récession économique en 1975 ne démarque pas le frein de la croissance du "suburbio" La "vague de peuplement" continue. probablement par les effets de la diffusion à l'intérieur du pays et aux pays voisins d'une image cosmopolite et d'accès aux opportunités d'emploi les plus abondantes et variées. 
Pour un journalier d'une exploitation agricole ou pour un employé municipal d'un village de l'intérieur, la possibilité d'"accéder" à la ville, grâce à l'acquisition d'un lot économique à deux heures du centre-ville; la construction d'un logement précaire; et la possibilité de travailler comme ouvrier de la construction, cueilleur de cartons et de bouteilles dans les rues, dits "cartonero" ou "botellero", ou travailleurs à leur compte; toutes ces situations sont plus attirantes que de rester avec la monotonie et le manque d'horizons depuis leurs endroits d'origine. Dans ces décisions, n'intervient pas le panorama de récession que les chiffres et la réalité même montraient entre 1976 et 1984 .

Ce phénomène social, reproduit en grand nombre dans la région métropolitaine, surtout depuis la decennie de 1940, enregistre deux manifestations territoriales différenciés.

D'une part, l'accession au lot propre sans services dans les endroits les plus périphériques de la région, à travers des systèmes de paiement en quote-part. C'est le cas typique de la micro-région.

D'autre part, l'occupation ilégale des "interstices" et des friches urbaines et suburbaines, fiscales ou privées, avec des niveaux d'accès assez bons, donnent lieu à l'émergence de bidonvilles appelées "villasmiseria" ; et plus tard aussi, à des emplacements spontanés et des lotissements clandestins, dits "asentamientos"

Conforme aux tendances globales mentionnées, la décennie de 1970 est celle d'une grande croissance démographique pour la micro-région, particulièrement dans les lotissements populaires. La localité de "Ingeniero Allan" passe de 5.000 habitants en 1970 à 12.500 en 1980. En fonction des données récentes on estime que s'est produit une stagnation du nombre d'habitants depuis 1983-1984. Pour 1991, la localité enregistre 17.300 habitants. Le peuplement pendant la décennie de 1980 est dû à deux interventions: l'inauguration d'un ensemble de logement en propriété horizontale à "Barrio Parque" pour un peu plus de 400 familles; et un "asentamiento" spontané depuis 1983 qui reçoit 450 familles, la plupart du nord-est argentin.

Cette croissance se traduit par une dégradation des conditions de vie, mise en évidence par le manque chronique d'accession aux services essentiels. La localité a $130 \mathrm{~km}$ de rues, dont $4 \%$ en est pavée. La fermeture du chemin de fer en 1977 a contribué à en déteriorer l'accès.

Pendant le gouvernement militaire, l'une des formes de vie les plus communes des nouvelles familles était l'occupation comme ouvriers des grandes investissements urbains: infrastructure routière et d'autoroutes, 
édifices bancaires et financiers, stades sportifs pour le championnat di monde de football. Les fils aînés accompagnaient généralement leur: pères comme aides de travail.

Dans la mesure où la crise augmente, la situation change. L'abscence de toute la famille durant le jour est évidente: les mères s'occupent de: tâches ménagères chez des familles de hauts revenus à Buenos Aires or aux sous-centres importants du sud métropolitain. Les week-ends, la localité prend vie à nouveau: on observe l'extension de la journée ds travail due à la construction qui n'est jamais finie de leur logement, ains qu'à divers travaux ménagers d'entretien.

La déterioration du salaire et le coût relatif élevé du transport déterminent des situationes extrèmes: Un habitant de cette localité aver un travail à Buenos Aires, consomme en transport jusqu'au $45 \%$ de sor salaire et $35 \%$ des heures du jour.

On identifie souvent la modification des formes de vie, imposée par le: circonstances. Face au manque total d'une occupation occasionelle à li ville, le père reste souvent chez lui avec ses enfants cadets, tandis que li mère s'emploie comme domestique -avec le transport payé- à la ville dı Buenos Aires; et les enfants aînés déambulent par les rues en vendan des petits objets de consommation.

On estime qu'entre 1970 et 1991 les lots occupés à Ingeniero Allaı passent de 1200 à 4000. Donc plus de 7000 lots, restent vides. Li transformation territoriale depuis 1975 signifie l'accéleration de li tendance d'occupation des lotissements populaires. Cependant, ceux-c restent encore dépeuplés; les investissements en services sociaux son difficilement réalisables.

\section{e) Les résidences secondaires et l'espace de loisir.}

A partir 1975, se présente une tendance de valorisation de la micro région comme espace de loisir et récreation par des secteurs de revenu hauts et moyen-hauts.

La "territorialisation" des polarisations sociales qui travers l'Argentine, produit des formes de ségregation territoriale marquée dans la micro-région. (FIGURE 29)

De nombreux fractionnements ruraux petits et des subdivision urbaines en grands lots, non-occuppés pendant une ou deux décennies 
commencent à être l'objet d'une commercialisation à travers des agences immobilières à Buenos Aires, Avellaneda ou Quilmes.

A cette situation, s'ajoute l'application en 1978 d'une loi qui promeut les phénomènes de ségrégation, à travers l'énonciation de la figure du "club de campo" ou "country" L'importance du thème est telle qu'on lui consacre un chapitre spécial avec six articles de la Loi Provinciale 8912/77 de "Ordenamiento Territorial y Uso del Suelo"

Le "club de campo" est un terrain situé dans une "aire non-urbaine" avec des logements en grands lots et un endroit commun destiné à la prestation de services centraux. Parmi d'autres conditions, la Loi établit que "...la frange du périmètre devra être boisée" (art.65); et que "...on pourra accorder avec la municipalité respective la fermeture totale de l'aire" (art.67); cela constitue une forme de ségrégation, à ce qui s'ajoute souvent des difficultés d'accès des "organismes publiques dans l'exercice de leur pouvoir de police" (art.67).

Il existe deux "clubs de campo" dans la micro-région: "El Pato" et "Los Tordos" avec 305 et 50 propriétés respectivement. Ces clubs prolifèrent par le nord-ouest de la région métropolitaine, sur des routes et autoroutes d'accés aux centres et sous-centres de plus grand pouvoir d'achat de la région métropolitaine.

Les autres trois formes caractéristiques de la région, et présentes dans la micro-région sont connues comme: quartiers-parc dit "barriosparque", lotissements pour résidences de week-end et fractionnements en parcelles rurales petites pour des villas, dit "casas-quinta" Les premiers, n'ayant pas les services centraux du "country", ont des systèmes de sécurité privée et quelques formes de fermeture du terrain. Les autres constituent des subdivisions urbaines en parcelles de grande taille; les deux se concrétisent par le cadastre à travers des îlots, appelés "manzanas", et certains îlots plus grands, les "quintas amanzanadas", situation qui suppose une réseau assez abondant de rues intérieures. Les "casas-quinta" sont des subdivisions un peu plus grandes, en parcelles de plus grande taille et souvent caractérisés par la présence de petits producteurs horticoles et floricoles.

On a enregistré dans la micro-région plus de 3200 grandes parcelles urbaines et 950 petites parcelles rurales, de moins de 2 ha. On remarque deux lotissements urbains: "Barrio Raelwi" à Berazategui: 779 lots; et "Barrio Ruta-Sol" à La Plata: 826 lots; et deux subdivitions rurales: "La Carolina" à F.Varela: 435 parcelles de 1 ha chacune; San Pablo" à Berazategui: 379 parcelles de $4.000 \mathrm{~m} 2$ de superficie moyenne. 
Cette capacité potencielle d'occupation d'à peu près 4500 parcelles urbaines ou rurales pour le loisir, se mobilise depuis les changements économiques et sociaux produits par le gouvernement militaire en 1976.

Bien que pendant la démocratie les formes de participation augmentent, la tendance de differenciation sociale continue. De nombreuses familles d'entrepreneurs industriels et financiers, de commerçants, de fonctionnaires et quelques professionnels, accèdent a des résidences-secondaires situées à une heure de la ville. Généralemenı se produit l'installation de toute la famille pendant l'été en coincidence avec les vacances scolaires des enfants, tandis que le père réalise tous les jours le voyage au travail.

Ces familles procèdent pour la plupart du sud de la ville de Buenos Aires, de Quilmes, de Avellaneda et de Lomas de Zamora.

L'augmentation des niveaux de chômage et les vols aux résidences non-habitées la plupart de l'année, provoquent une situation nouvelle. I] s'agit des nombreux emplois occasionnels pour des travaux de nettoyage pour les femmes, et en jardinerie et entretien des propriétés, pour les hommes chômeurs. Malgré les mauvaises rétributions, le grand épargne en transport à Buenos Aires, devient une alternative possible dans le contexte de crise en vigueur.

La transformation territoriale récente intervient en augmentant la ségrégation socio-spatiale, mais aussi en donnant lieu à l'articulatior inégale dans les relations de travail.

\section{f) La crise maraîchère.}

Les déterminants de cette crise sont liés à l'accentuation de lá tendance récessive à un niveau global: il y a une réduction de la taille di marché intérieur. Les manifestations sont assez diverses: les hauts coûts des matières et des technologies étrangères; l'inutilisation croissante des capacités installées; les difficultés dans la commercialisation et les meilleures attentes dans d'autres activités.

L'une des stratégies développés à partir de la crise de la fin des années soixante-dix est la diversification pour subsister. Les floriculteurs ou les horticulteurs, commencent d'autres activités: li aviculture, la grange, l'élevage de porcs et l'apiculture.

Vers 1988-1990 le territoire des productions intensives s'ètai notamment réduit. Des couvertures floricoles et des exploitation: 
horticoles abandonnées sont la manifestation du recul notable de la production.

Les lotissements attribués par la "JICA", l'Agence de Coopération Internationale du Japon, en 1987 à 33 familles à la Colonie La Plata II sont rapidement dépeuplés. En 1989 il n'y avait plus que 4 familles japonaises (Tadeo; 1991:32) (FIGURE 30)

La diminution de la rentabilité horticole entraine l'accentuation des niveaux d'exploitation des travailleurs, et la disparition des établissements comme tels. Dans des interviews réalisées en 1988, on a identifié de nombreux hommes qui en travaillant avec leurs familles percevaient des sommes equivalentes à 1 ou 1,50 dollar par jour. (H.Bozzano et P.Pintos)

Les conditions de travail des "medieros" sont, souvent, infra-humaines. $\mathrm{Ce}$ sont pour la plupart de nouveaux migrants qui procèdent des provinces du nord argentin, de la Bolivie et du Paraguay. Leur logement est de tôle démontable avec des latrines. La provision de la nourriture essentielle pour la subsistance est réduite du salaire.

L'autre gros problème est celui de la commercialisation des fleurs, fruits et légumes. 95\% de la production va au "Mercado Concentrador de Buenos Aires" où les vendeurs de fleurs obtiennent des bénéfices au détriment des producteurs, en profitant souvent des problèmes linguistiques rencontrés par les Portugais et les Japonais.

La commercialisation de fruits et légumes réunit un plus grand nombre de personnes. L'intermédiation, connue comme "consignación" est le principal facteur de réduction des profits. L'ouverture du "Marché Central de Fruits et Légumes" à La Matanza ne constitue pas une vraie solution. Il faut une recherche profonde pour améliorer cette question.

En ce qui concerne l'innovation à l'intérieur des circuits productifs, le rétard en est rémarquable, sauf dans le cas des grands producteurs. Ce sont pour la plupart de petites exploitations.

Les petites exploitations horticoles, de moins 5 ha, se caractérisent pal un "...retard structurel défini par une organisation qui répond à ses deux seules ressources: l'homme et la terre; avec une faiblesse de négociation' (De Biassi et Gratti;1990)

Les petites exploitations floricoles, de moins 2 ha emploient unt technologie simple dejà désuète: l'absence de locaux vitrés ou plastifiés pour les plantes, dits "invernáculos" plus modernes, de chambres 
froides, d'équipes d'arrosement par aspersion les machines sont anciennes; les producteurs réproduisent des pratiques traditionnelles fondés sur la connaissance empirique.(Tadeo; 1991:36-37)

\section{g) Les mutations aux productions intensives.}

Les mutations des productions intensives traditionnelles partent de la différentiation à l'intérieur des circuits économiques horticoles et floricoles.

La consolidation des grands producteurs provient principalement du contrôle des cannaux de commercialisation et par la capacité de réinvestissement dans l'activité. Dans la plupart des cas cette situation suppose l'existence d'un début dans cette activité, dans des conditions économiques plus avantageuses.

La grande exploitation horticole périurbaine est normalement un ensemble discontinu de nombreuses petites exploitations, où chacune fonctionne avec une famille en "mediería", en empêchant de cette manière d'avoir un endroit naturel de discusion des groupes ayant les mêmes conditions de vie.

Les grands producteurs horticoles "...ont assimilé des nouvelles pratiques dans l'organisation de la production; ils ont une haute capacité d'accumulation; et ils sont poussés par d'autres intérêts: la recherche de qualité, la valorisation de leurs produits et la recherche du marché extérieur. Ils ont une organisation hiérarchisée et salariée du travail, avec l'incorporation technologies non-traditionnelles et l'organisation permanente entre des producteurs" (De Biassi et Gratti; 1990)

Les producteurs floricoles les plus consolidés ne peuvent pas concurrencer encore les marchés internationaux. Malgré certains progrès technologiques, comme la chaîne de froid, l'adaptation à des critères de qualité du produit et des contrôles phyto-sanitaires internationaux le marché argentin d'exportations de fleurs n'est pas d'importance. Dans ce cadre il est presque impossible de s'immiscer dans les centres de distribution de l'Hollande ou nord-américains.

Il y a eu récemment des expériences ponctuelles de réconversion productive, assez significatives. Parmi d'autres, on rémarque: l'utilisation de couvertures plastiques et l'introduction de nouvelles productions à la région. 
Bien que la diffusion des couvertures plastiques pour certains légumes est récente, il existe une tendance de croissance rapide. Les principaux avantages sont l'augmentation de la productivité, l'amélioration de la qualité pour s'introduire dans les marchés internationaux, et la possibilité de produire à n'importe quelle saison de l'année. La limitation concerne la disponibilité de capital initial; cependant, les profits sont. selon les interviewés et le type de produit, entre deux et sept fois supérieures à ceux des exploitations traditionnelles.

Actuellement on assiste á une mutation productive considèrable. I] s'agit de l'introduction de cultures commerciaux de "kiwi" et l'élevage de loutres, lapins et grenouilles. Les stations agraires-experimentales de l'Université National de La Plata à Gorina et de l'INTA, l'Institut National de Technologie Agropastorale à F.Varela; et l'Ecole de Cunniculture el Apiculture de la Province de Buenos Aires à La Plata, réalisent des recherches et diffuse leurs travaux de manière croissante. L'obtention de nouvelles variétés d'espèces végétales (tomate, melon, courge, fraises, concombres, etc) et l'appui à leur diffusion dans la zone sont des plus remarquables.

Cettes mutations productives récentes interviennent comme déterminants des transformations territoriales. L'espace des exploitations à ciel-ouvert donnera lieu pendant la prochaine décennie, à l'alternance et à la juxtaposition du traditionnel agraire -exploitations un peu plus grandes- et du nouveau, plus intensif et de moindre taille. 


\section{FIGURE 28}

\section{LE RELIEF DANS LA MICRO-REGION DE INGENIERO ALLAN-EL PATO-EL PELIGRO}

LA PLUPART DE L'AIRE SE TROUVE ENTRE 25 ET $30 \mathrm{M}$ DE HAUTEUR. VERS LE NORD ON IDENTIFIE LES VALLEES DE "PEREYRA" ET DE "CONCHITAS" ENTRE LES COURBES DE NIVEAU DE 20 ET $10 \mathrm{M}$ : IL S'AGIT DES TROIS VERTS LES PLUS FONCES.

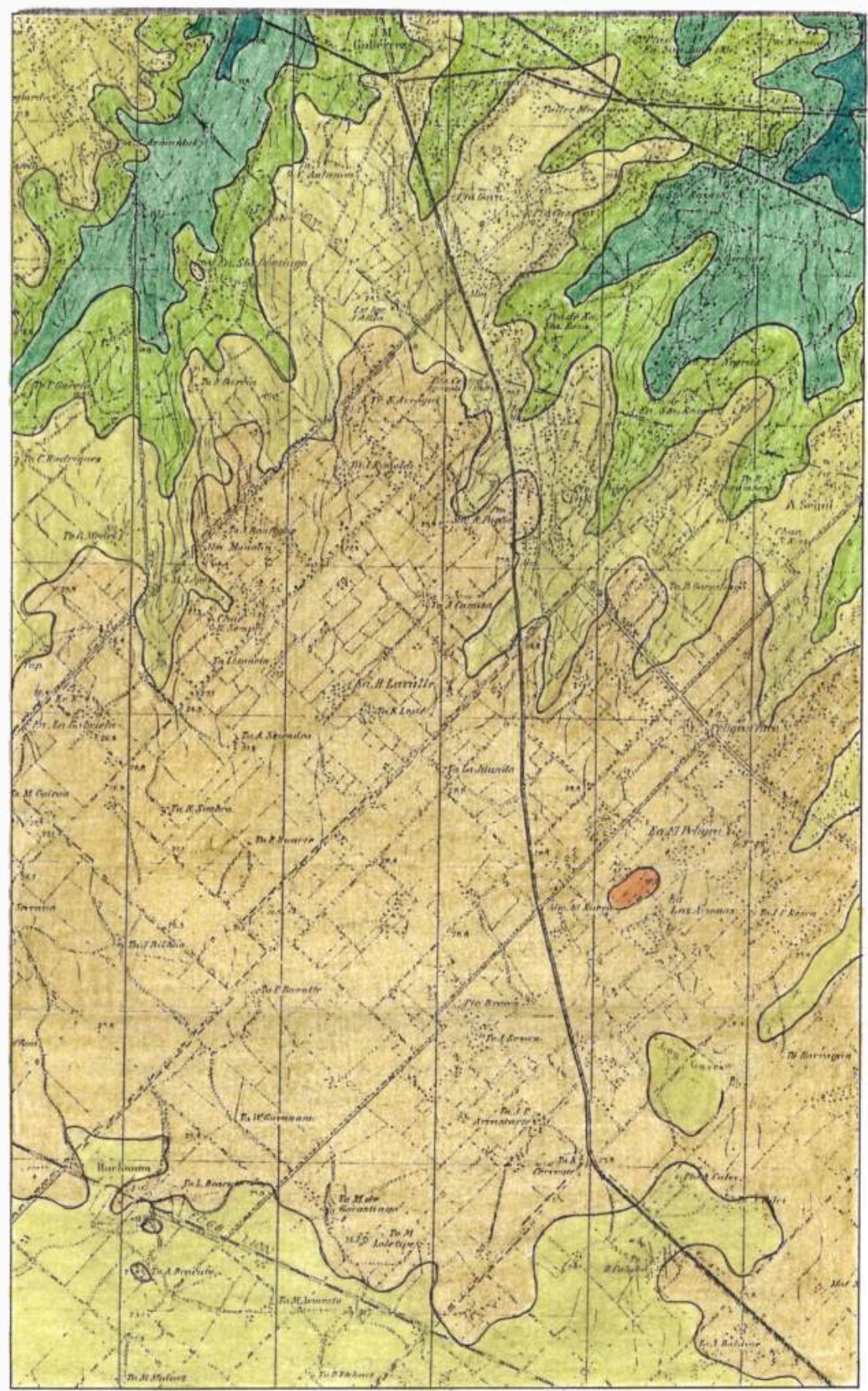

Source: Elaboration personnelle à partir de la Carte Topographique de l'Institut Géographique Militaire Argentin. 
FIGURE 29

\section{L'USAGE DU SOL DANS LA MICRO-REGION DE INGENIERO ALLAN-EL PATO-EL PELIGRO}

(ELABORATION PERSONNELLE A PARTIR DE PHOTOGRAPHIE AERIENNE DE 1984)

ESPACE URBAIN RESIDENTIEI.

PLUS 50\% ESPACE OCCUPE
20/50\% ESPACE OCCUPE
MOINS 20\% ESIPACE OCCUI'E

ESPACES VACANTES OU NON-UTILISEES

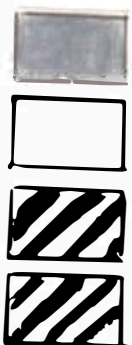
ILOTS

PARCELLES RURAI.ES

FRICHES INDUSTRIELLES

FRICHES MARAICHERES
ESPACES PRODUCTIFS

\begin{tabular}{ll} 
INDUSTRIE \\
HORTICULTURE \\
FLORICULTURE \\
AVICULTURE \\
CARRIERES \\
\hline
\end{tabular}

\section{ESPACES DE RECREATIQN}

PARCS/VILLAS/"CLUB DE CAMPO"

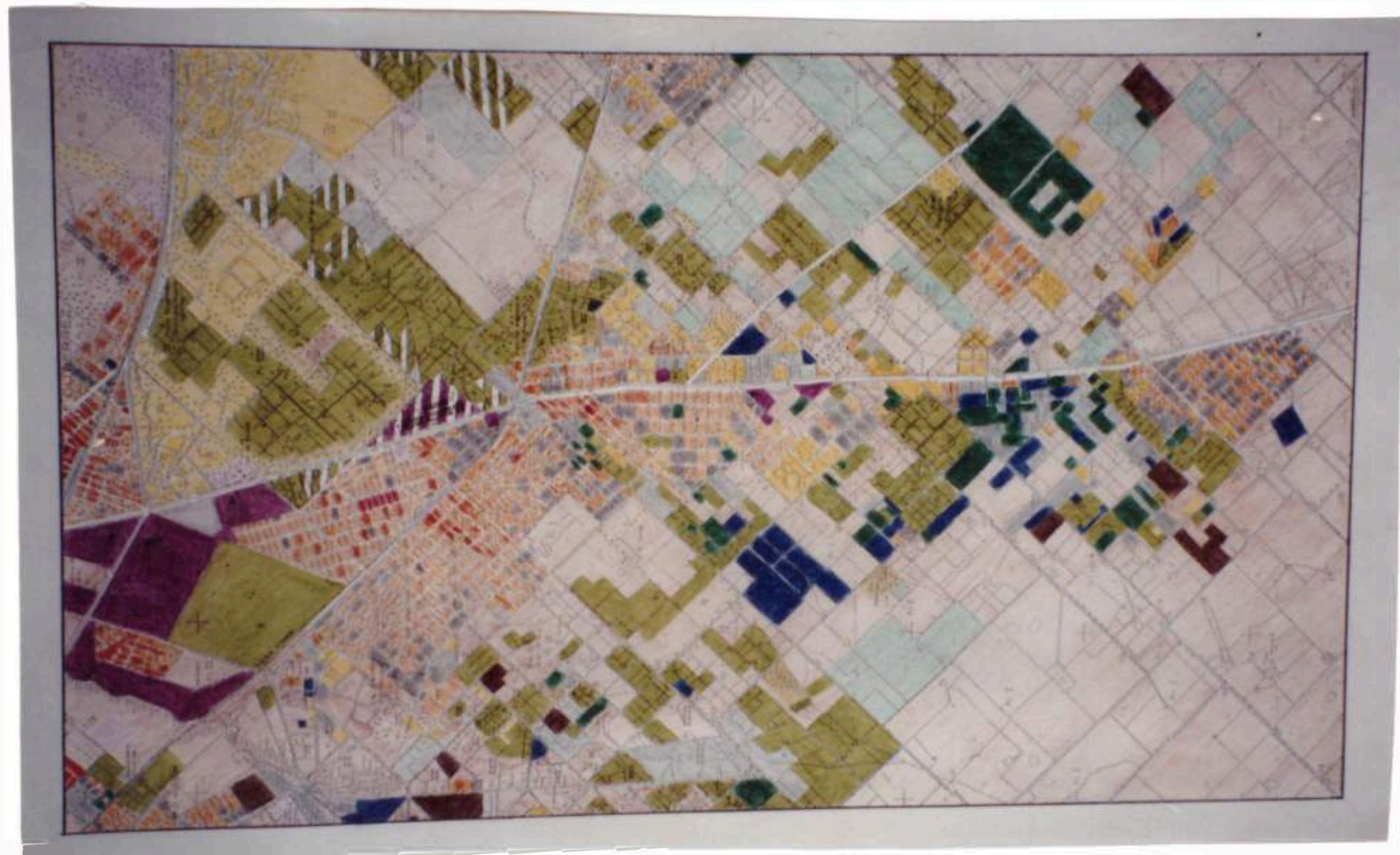

114 
FIGURE 30

LA MICRO-REgION DE ING.ALLAN-EL PATO-EL PELIGRO

LES PRODUCTIONS INDUSTRIELLE ET AGRICOLE INTENSIVE

ON PEUT IDENTIFIER UNE "COURONNE DE PRODUCTIONS INTENSIVES" QUI ENTOURE LES LOTISSEMENTS TOUT LE LONG LA ROUTE NATIONALE 2. LES INDUSTRIES SONT DANS UNE POSITION PLUS AVANTAGEUSE QUE L'AIRE MARAICHERE.

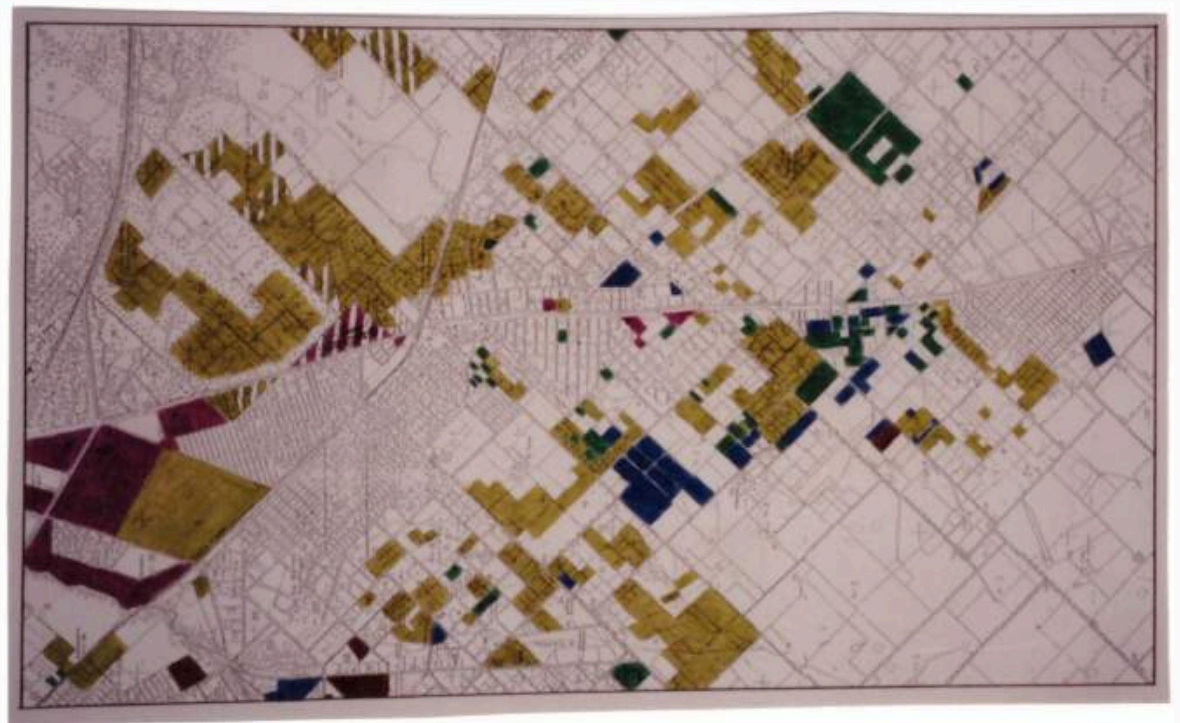

\section{FIGURE 31}

\section{LA MICRO-REGION DE ING.ALLAN-EL PATO-EL PELIGRO LES ESPACES PRODUCTIFS}

PLUS DE 80\% DU TERRITOIRE EST PRODUCTIF. IL EXISTE MEME DES PRODUCTIONS INTENSIVES DANS CERTAINS LOTISSEMENTS URBAINS. A LA CEINTURE MARAICHERE, SUCCEIJE L'AIRE D'AGRICULTURE COMMERCIALE ET D'ELEVAGE PROPRE DE LA "PAMPA"

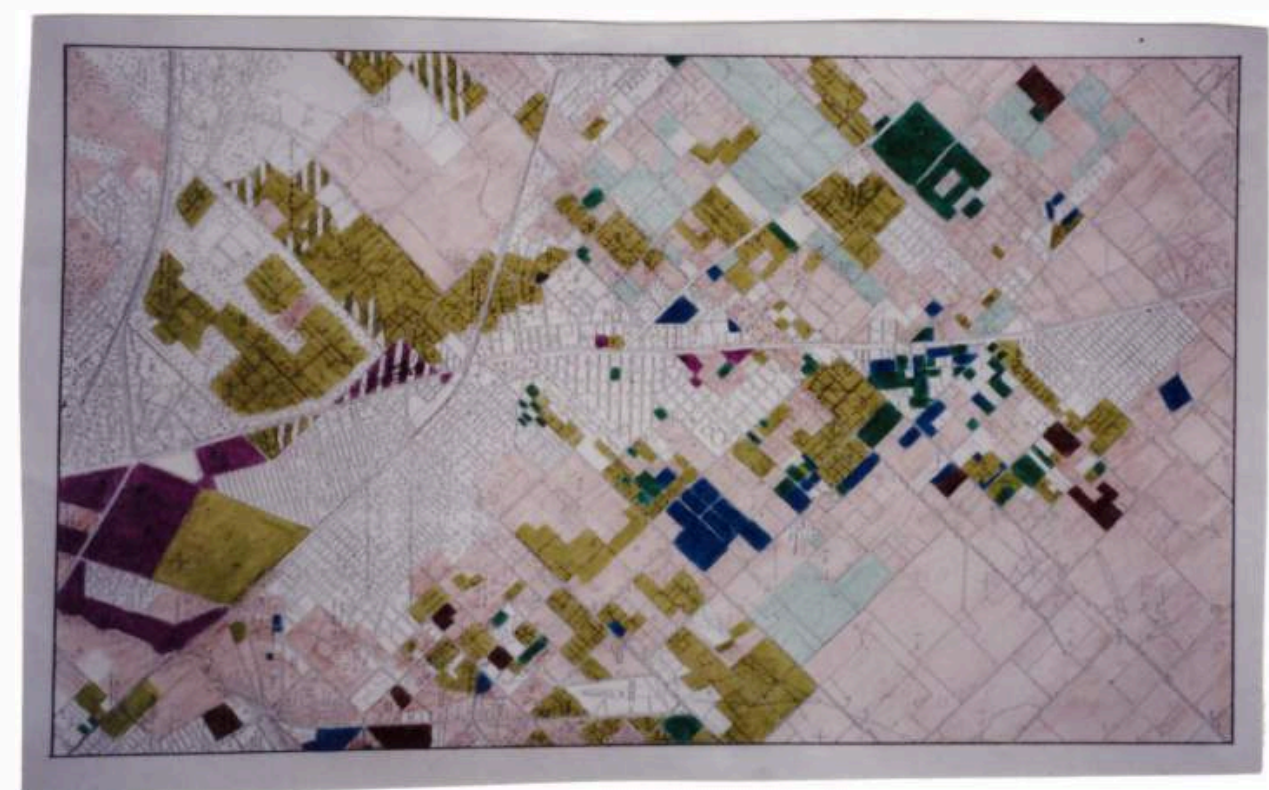




\section{FIGURE 32}

LA MiCRO-REgiON DE ING. ALLAN-EL PATO-EL PELIGRO EVOLUTION DE LA SUBDIVISION DE LA TERRE

\section{LOTISSEMENTS URBAINS}

PETITES SUBDIVISIONS RURALES

(PARCELLES INFERIEURES DE 5 HA)

$1945 / 1951$

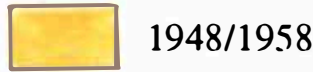

$1952 / 1968$

$1958 / 1990$

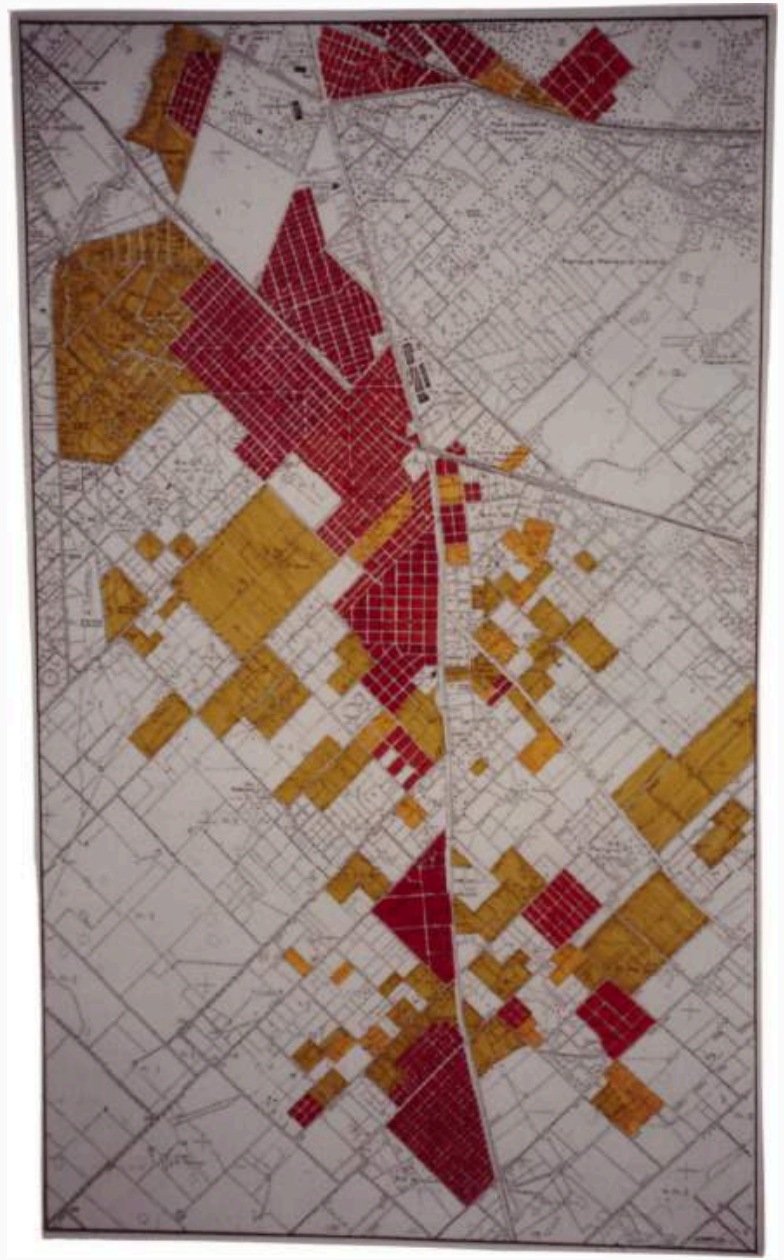

Sources: Elaboration personnelle à partir de: a) Municipalidad de Berazategui (1990) Evolución de la subdivisión de la tierra; el b) Peirano,G. A.González et H.Bozzano. Op.cit. 
FIGURE 33

LE "CENTRE INDUSTRIEL ROUTE 2 CIR 2" DANS L'ANCIENNE USINE "PEUGEOT", EN COURS DE RECONVERSION PRODUCTIVE.

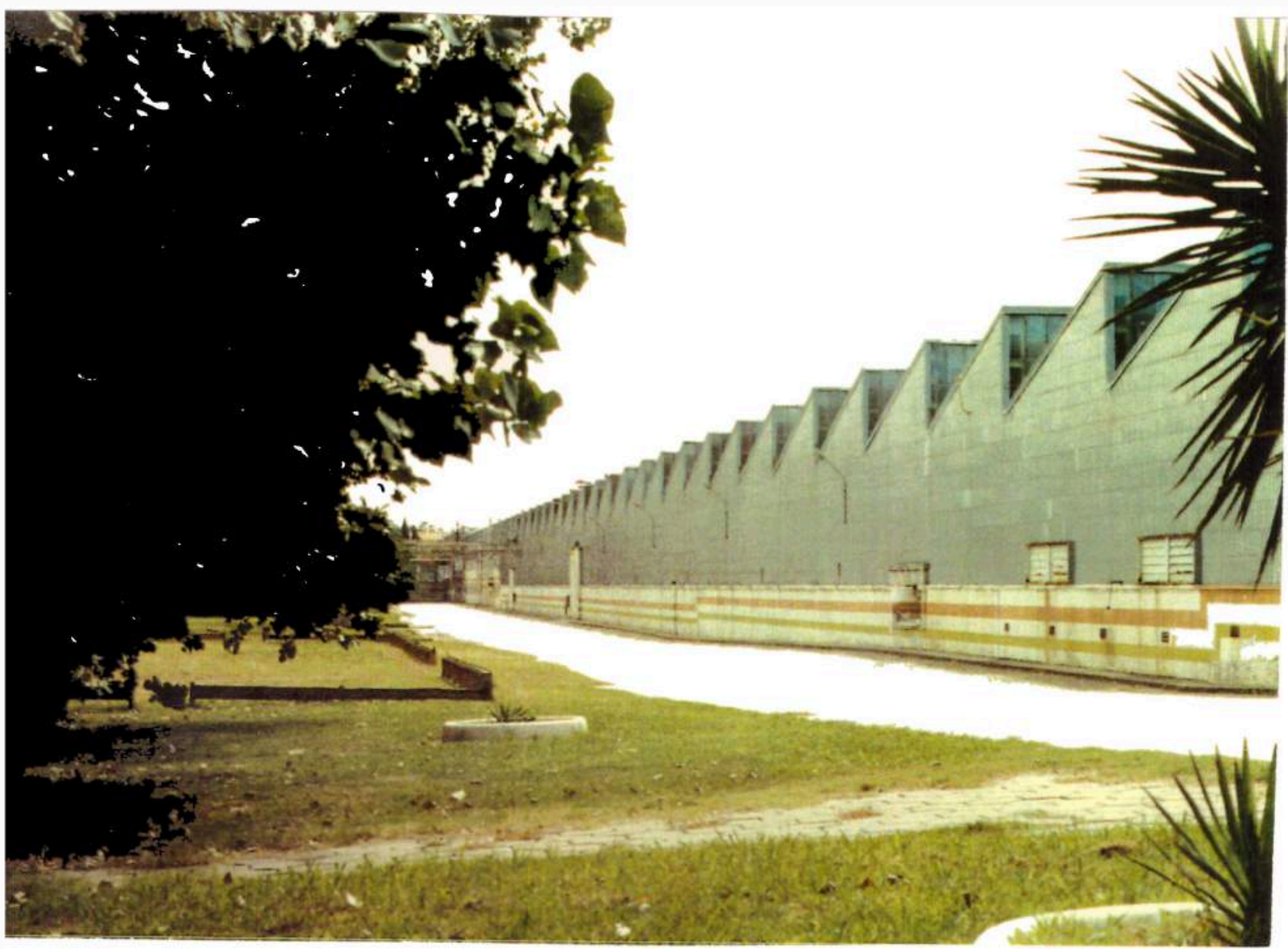

Photo: Courtoisic du "CllR2-Centro Industrial Ruta 2" 
FIGURE 34

L'INTERIEUR DE L'USINE "PEUGEOT", REFONCTIONNALISE EN 1989, EN COURS DE RECONVERSION TECHNOLOGIQUE ACTUELLEMENT.

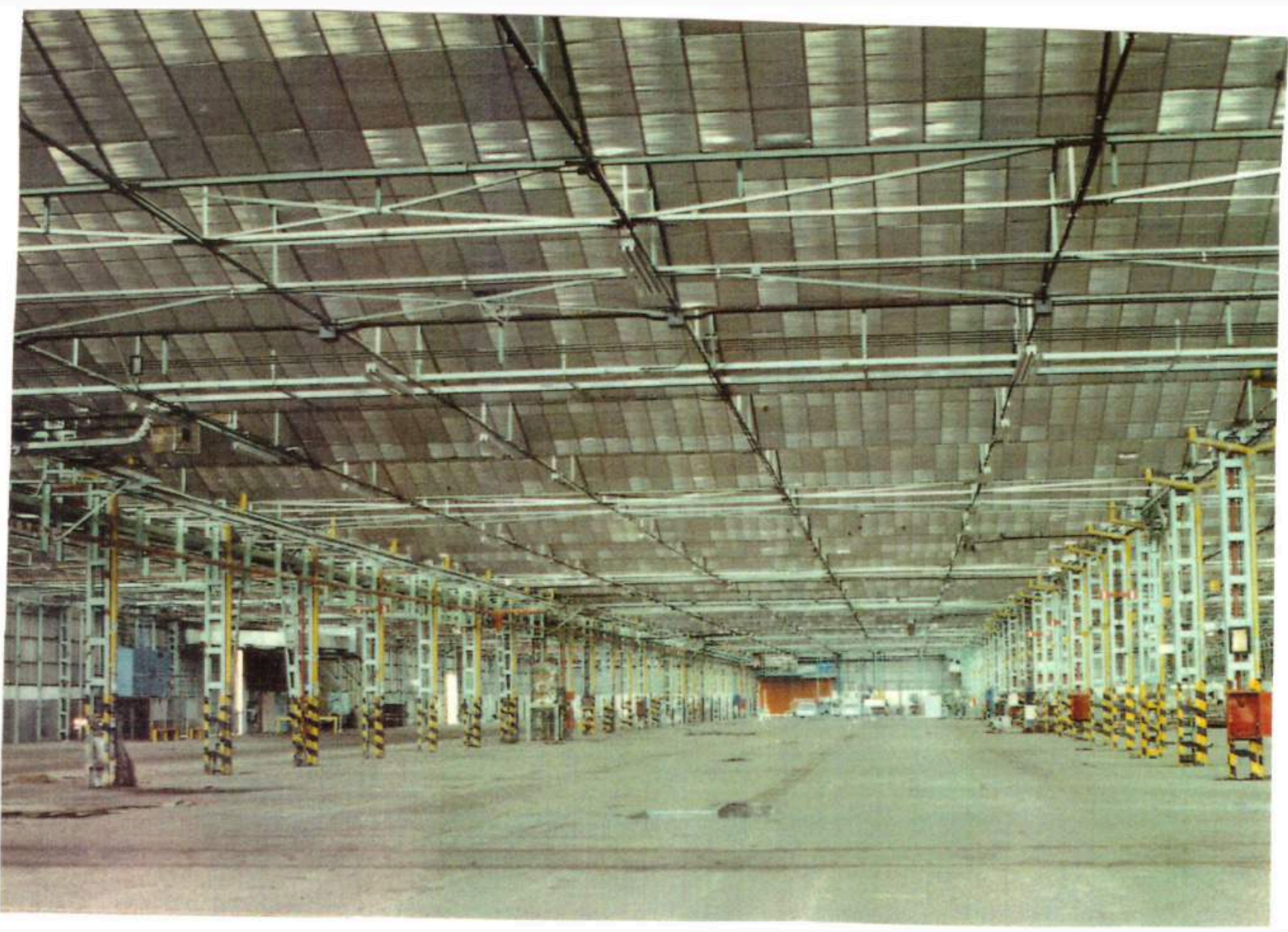

Photo: Courtoisie de "CIR2-Centro Industrial Rula 2" 


\section{V - CONCLUSIONS}

A partir de l'analyse des transformations territoriales et des mutations industrielles et agricoles dans la Région Métropolitaine de Buenos Aires, il est possible de tirer de nombreuses conclusions.

Le traitement à différentes échelles -la région et les micro-régionscontribue à approfondir l'étude de l'objet, soit comme "processus", soit en ce qui concerne ses manifestations ponctuelles. Ce travail essaie de trouver les relations entre des situations de transformation et de mutation générale et la forme qu'elles présentent à l'échelle locale.

L'organisation des conclusions, donc, se réalise: a) en fonction de la nature des mutations industrielles ou agricoles et b) en fonction du niveau d'approximation générale (à travers des processus); ou ponctuelle (à travers l'analyse des acteurs).

Il s'agit de 1-Territoire et production industrielle 2-Territoire et production agricole intensive et 3-Territoire, production et acteurs.

\section{1 - TERRITOIRE ET PRODUCTION INDUSTRIELLE.}

a-Les mutations industrielles dans la région métropolitaine sont en rapport avec des modèles économiques dominants en Argentine.

La succesion des modèles qui ont privilegié des économies agropastorales, industrielles et financières, se manifestent sur le territoire métropolitain. Les "saladeros" et les frigorifiques de la région proviennent de modèles agro-pastoraux. Les deux périodes de substitution d'importations prennent cette région de manière dominante pour se déployer. La désindustrialisation et la "financiérisation" de l'économie donnent lieu à un nouveau scénario dans la région. La reconversion productive actuelle choisit cette région pour concentrer la plupart des réseaux de connaissance, d'informations et de technologies. 
b-Les transformations territoriales à la régior métropolitaine s'accélèrent à partir des périodes de croissanc d'emploi industriel.

Chaque période de croissance d'emploi industriel est suivie d'unt expansion urbaine explosive. Premièrement, à partir de la spécialisatior du territoire à Barracas-Avellaneda-Lanús ; à Berisso-Ensenada ; et dans certains quartiers de la Capitale Fédérale. Durant ce siècle, des lotissements au delà de l'installation d'industries la première couronne entre 1915 et 1945 et certains axes et la deuxième couronne, entre 1945 et 1965. Le premier cas donne lieu à la formation d'un tissu mixtc résidentiel-industriel. Pendant ce siècle le lotissement entoure l'établissement et la station ferroviaire, mais la plupart se situe er dépassant les nouvelles usines, en faisant croître la périphérie urbaine significativement.

c-Les dernières localisations des grandes industries à la périphérie pendant les décennies de 1940 et de 1950 : n'arrêtent pas le rythme d'accroissement de lotissements plus périphériques.

La nécessité de grands espaces pour des grandes usines contribue à développer une tendance de localisation des industries dans des zones presque rurales. L'usine "Peugeot" à "El Pato" en est un exemple, où se produit la prolifération des lotissements au delà l'établissement, effel mésurable encore neuf ans après.

d-La proximité des industries et des lotissements contigüs n'est pas signe d'une relation directe et dominante en matière d'emploi et de résidence.

A l'exception des périodes où les moyens de transport étaienı primitifs, la localisation de proximité entre l'usine et la résidence n'étail pas absolue. Le développement des réseaux de transport public assez effectifs et accesibles contribue à relativiser ce rapport. Pendant les périodes de substitution d'importations, les industries se trouvent plus éloignées du centre-ville que les endroits de résidence ouvrière. Les usines "Alpargatas" et "Peugeot" emploient habitants des villes et des quartiers plus proches de Buenos Aires: Quilmes, Berazategui, Avellaneda. 
e-Les logiques qui président à l'appropriation d'espaces périphériques varient selon s'agit de l'industrie ou de la résidence.

Bien qu'il s'agit pour ces deux termes d'une question de disponibilté de terrain, les logiques sont diverses. Tandis que l'industrie a besoin de grandes parcelles inexistantes dans la ville ou le "suburbio" ; il subsiste de nombreux lots suburbains inoccuppés ; mais le coût de la terre la rend inaccesible pour les nouveaux migrants à moindres ressources ils doivent donc acquèrir terrains situés dans des aires rurales.

f-La reconversion industrielle tend à profiter des avantages comparatifs de la région métropolitaine par rapport à d'autres régions d'Argentine.

L'existence des centres de recherche et d'excellence les plus importantes d'Argentine, dans la Région Métropolitaine de Buenos Aires, est le facteur déterminant d'une future territorialité de "pôles" et de "technopoles" Les exemples des Pôles Petrochimique de Ensenada et Informatique de Berisso sont actuellement les plus saillants. Ils fonctionnent avec des avantages de complémentarité entre entreprises, en profitant à la fois de la croissante relation université-entreprise.

\section{g La reconversion industrielle tend à produire un décalage plus grand entre les usines et les localités où elles se trouvent, en provoquant des comportements d'"enclave".}

La reconversion technologique actuelle a besoin de personnel de haute qualification, souvent des professionnels, qui n'habitent pas près des usines et des pôles. La plupart des employés des enterprises les plus automatisées et robotisées ne procèdent pas des localités où celles-là se trouvent. Voilà le cas de "Petrokén" avec Ensenada et des enterprises du Pôle Informatique avec Berisso. Il existe des élements pour affirmer que la même situation va se répéter à "Sevel/Fiat-Peugeot" avec El Pato et Ingeniero Allan. 
a La majeure expansion d'espaces agricoles périurbains coïncide avec des périodes de plus large accroissemen1 économique global et d'expansion du marché de consommation interne.

Le ceinture maraîchère de la région métropolitaine se répand plus notablement pendant les années 1910, 1920, 1940 et 1960, coincidan 1 avec les périodes d'expansion économique globale. Dans tous les cas, il $n$ y a pas de mutation productive importante le rapport "terre-travail" est le facteur déterminant de cette activité.

b - Les productions agricoles intensives ont été déplacées: pas toujours dans les directions des axes de croissance.

L'expansion urbaine a déterminé le déplacement des productions intensives et la spécialisation de territoires durant certaines périodes. Des secteurs anciennement urbanisés du nord de Buenos Aires (San Isidro), on passe à ceux de l'ouest (Merlo, General Sarmiento et Morón) et du sud-est (La Plata et Berisso). Les deux zones les plus importantes pendant les quatre dernières décennies sont le sud-est (La Plata, Berazategui et Florencio Varela) et le nord-ouest (Escobar et Pilar).

c - La différentiation de groupes selon leurs niveaux technologiques de production ne produit pas de ségregation spatiale, mais la sujétion ou la margination dans des phases diverses des circuits économiques respectifs.

L'incorporation de nouvelles technologies de production n'a pas de relation avec la localisation de chaque producteur. Elles se présenteni juxtaposées à des producteurs traditionnels, donc il n'existe pas de ségregation spatiale. La possibilité d'avoir des technologies signifie une amélioration de la productivité cette amélioration se traduit, ensuite, dans une meilleure insertion dans les phases de commercialisation du circuit correspondant, soit horticole, soit floricole.

d La présence de groupes de producteurs agricoles intensifs dans des espaces contigüs et adjacents, tend à produire les situations de permanence les plus remarquables dans la périphérie métropolitaine. 
La consolidation d'espaces homogènes en ce qui concerne la production agricole intensive, malgré des différences de taille d'exploitation, contribue à donner lieu à des territoires qui ont pu subir l'influence de la croissance urbaine. Les cas les plus saillantes sont ceux des colonnies agricoles créees à une telle fin; dans la micro-région il s'agit des exploitations fractionnées et appropriées durant la même période: les Portugais à El Pato et les Japonais à El Peligro. Dans cettes situations, la permanence territoriale ne signifie ni une "ankylose productive" ni la non-introduction des nouvelles technologies.

e Dans la répartition des espaces agricoles, la différentiation entre les producteurs traditionnels de la "pampa" et les producteurs d'agriculture intensive est principalement dûe à des gradients des rentes différentielles agraires.

L'agriculture intensive périurbaine a une productivité plus importante que sa voisine de la "pampa" proprement dite. La productivité est en rapport avec la nature capital-intensif de l'exploitation, soit en machines, soit en technologie, soit en travail. La composant du coût de transport dans la rente différentielle, jusqu'à quarante ou vingt années en arrière, était déterminante, selon les produits. Pour une ou autre raison, la différentiation des systèmes productifs agricoles entre les régions métropolitaine et la "pampa" a toujours été une question de gradient de rentes agraires, même en considérant sa juxtaposition à des gradients de rentes urbaines.

f L'introduction de mutations technologiques à la production agricole intensive tend à s'accélérer actuellement à cause du processus de diffusion internationale de biens alimentaires de consommation.

Deux ou trois décennies après, la consommation d'aliments dans chaque société se réduisait à un nombre determiné de produits. Actuellement l'augmentation du nombre de biens de consommation alimentaire, d'une part et la diffusion des innovations biogénétiques el des techniques de production, ont notamment changé le cadre des productions intensives. Le rapprochement entre la recherche agricole el l'application à l'exploitation démontrent la nature de la mutation productive actuelle. 


\section{3 - TERRITOIRE, PRODUCTION ET ACTEURS}

a Les périodes des mutations productives les plus profondes sont celles de plus importante hétérogénéité structurelle de la formation sociale.

L'introduction de mutations productives, quelles que soient leur nature et leurs caractéristiques, suppose une augmentation du décalage entre les acteurs directement concernés et les acteurs non-impliqués. La difficulté d'accès d'une grande proportion de la population à des niveaux de qualification et à l'utilisation des innovations tend à produire une forte différentiation sociale. Le cas actuel de Berisso est remarquable.

b - L'appui de l'Etat central à l'installation ou à la reconversion d'industries ne coincident pas toujours avec le pouvoir local, à cause des différents niveaux d'articulation aux acteurs locaux.

Bien que la génération d'emploi industrielle soit impérative, dans le cadre actuel de crise générale; dans certains cas la dévalorisation environementale du milieu urbain subit la résistance des populations locales concernées avec l'appui des gouvernements municipaux. Il y a deux cas éloquents à Ensenada et Berisso: respectivement les fabriques de coke résiduel et d'acide sulfurique.

c Les grandes enterpreneurs sont ceux qui bénéficient le plus des politiques d'appui aux investissements productifs en vigueur dans la région métropolitaine.

La politique nationale de privatisations et de concessions des entreprises publiques et d'infrastructures est un moyen de consolidation économique pour les grandes groupes. Les investissements productifs sont fréquemment promus à travers des mecanismes de capitalisation de la dette extérieure. La Province appuie l'installation d'industries à travers l'exemption de tous les impôts. Dans ce cadre on peut identifier, deux enterprises publiques en cours de privatisation: "Petroquímica General Mosconi" et "YPF"; et la dernière entreprise pétrochimique du Pôle avec des bénéfices des titres de la dette.

d Les stratégies de sous-emploi tendent à articuler de façon subordonnée les acteurs de la micro-région de Ingeniero Allan-El Pato-El Peligro ; particulièrement entre les secteurs 
des plus bas revenus, les propriétaires de résidences secondaires et les producteurs d'agriculture intensive.

La diffusion de la crise et le coût relative élevé de transport au centreville, contribuent à développer des stratégies de sous-emploi non qualifié de basse rémuneration. L'impossibilité de dépenser $45 \%$ du salaire en transport, induit des travaux dans des services de nettoyage, de jardinerie et de securité dans des résidences-sécondaires, et en moindre mésure, comme journalier dans des exploitations horticoles.

\section{REFLEXION FINALE}

Mutations productives: Objet de traitement dans l'étude des processus propres des pays centraux. Comment se manifestent-elles dans les pays périphériques ? L'un des aspects les plus saillantes en est précisement, la possibilité de trouver des analogies et des différences entre ces deux situations.

A partir de cette recherche on identifie des types de mutations productives l'une, porteuse et diffuseuse d'innovations, propre aux pays centraux; l'autre, qui reçoive ou qui acquiert ces innovations-là, après un temps de diffusion variable, ou qui les développe de manière indépendante, même en ayant un certain retard par rapport à l'innovation originale. On les appelera respectivement des "mutations innovatrices" et des "mutations résiduelles"

La tendance actuelle paraît indiquer le raccourcissement de temps entre l'application d'une mutation comme "innovatrice" et comme "mutation residuelle" Il existe une relation avec le temps de remplacement d'une technologie innovatrice par un autre plus nouvelle. Ces périodes sont de plus en plus courtes. Donc, il y a aussi une diminution des temps d'amortissements des innovations.

Dans ce contexte d'accéleration des innovations technologiques. Existe-t-il l'accéleration du rythme des transformations territoriales correspondante ? Le cas étudié paraît mettre en évidence un décalage entre la vitesse des mutations productives et la permanence du territoire. Tous les acteurs du territoire ne sont pas inclus dans les mutations productives. La transformation territoriale va vers une différentiation, due à ceux qui reçoivent et à ceux qui ne reçoivent pas les diverses mutations. 


\section{BIBLIOGRAPHIE}

AIT EL HADJ, Smail (1985a) "Aux origines des mutations technologiques" In: Les Cahiers Français. Mutations technologiques et formations. $\mathrm{N}^{\circ} 223$. La Documentation Française. Paris

AIT EL HADJ, Smail (1985b) ""De la société de la matière à la société de l'information" In: Les Cahiers Français. op.cit. Paris.

AZPIAZU, Daniel, E.BASUALDO et M.KHAVISSE (1986) El nuevo poder económico en la Argentina de los años ochenta. Legasa, Buenos Aires.

AZPIAZU, Daniel, E.BASUALDO et H.NOCHTEFF (1989) La Revolución Tecnológica y las políticas hegemónicas. El complejo electrónico en la Argentina. Chapitres I et V. Legasa, Buenos Aires

BIDAULT, Francis(1985) "Changements techniques et mutations de l'appareil productif" In: Les Cahiers Français...op.cit. Paris.

BORON, Atilio (1989) "Los actores y el libreto: Los sujetos sociales de la reconversion industrial en Argentina" In: Industria, Estado y Sociedad. La reestructuración industrial en América Latina y Europa.(pp.365-391) Eural. Nueva Sociedad. Caracas.

BOZZANO, Horacio et A.VIVES (1982) "Evolución del uso de la tierra en el Gran La Plata. 1580-1982" (pp.65-79) In: La Plata Una Obra de Arte. Posban \& Saltzmann, Buenos Aires.

BOZZANO, Horacio (1989) "Límites a la estructuracuión de espacios periurbanos: $i$ Legalidades naturales o efectos útiles de aglomeración ? El caso del Sureste de la RMBA"(pp.11-15). In: II Jornadas Regionales sobre Medio Ambiente. Facultad Cs.Naturales-UNLP. Municipalidad de La Plata.

BOZZANO, Horacio (1991) "Los procesos de estructuración de espacios periurbanos. Hacia una definición del borde metropolitano de Buenos Aires"(pp.264-284) In: Revista Interamericana de Planificación SIAP. Vol.XXIII-N89. SIAP, Guatemala.

CAPPANNINI, Dino et V.MAURIÑO (1966) "Suelos de la zona litoral estuárica entre las ciudades de Buenos Aires y La Plata" I.N.T.A., Buenos Aires

CORAGGIO, José Luis (1987) Territorios en transición-Chapitre I. Ciudad, Quito. 
CHIOZZA, Elena -direction générale- (1977) El País de los ArgentinosVolume VI "Región Metropolitana" Centro Editor de América Latina, Buenos Aires.

CHIOZZA., Elena (1983) "La integración del Gran Buenos Aires" In: Buenos Aires Historia de Cuatro Siglos (pp.421-449) Abril, Buenos Aires.

CHIOZZA, Elena -direction- (1985) Atlas Total de la República Argentina-Atlas Económico.Región Metropolitana. Centro Editor de América Latina CEAL, Buenos Aires.

CREVOISIER,O. et D.MAILLAT (1989) "Milieu, organisation industrielle et système productif territorial: vers une typologie" In: GREMI. IRER Neufchatel.

DE BIASSI, Mirta et P.GRATTI (1991) Plan de reactivación del sector hortícola: Una propuesta para pequeños productores. Departamento de Geografía, UNLP. La Plata (inédit)

DE MATTOS,Carlos (1990) "Reestructuración social, grupos económicos y desterritorialización del capital. El caso de los países del Cono Sur. In: Revolución tecnológica y reestructuración productiva Impactos y desafíos territoriales. Grupo Editor Latinoamericano, Buenos Aires.

DOLLFUS, Olivier (1976) L'espace géographique. Presses Universitaires de France, Paris.

DORFMAN, Adolfo (1982) Historia de la Industria Argentina. Solar, Buenos Aires.

EVERS, Tilman (1987) El Estado en la periferia capitalista. Siglo XXI, México (1ère édition allemande: 1977)

FIDALGO, Francisco et O.MARTINEZ (1983) "Algunas características geomorfológicas dentro del Partido de La Plata".(pp.263-279) In: Revista de la Asociación Geológica Argentina. Tomo XXXVIII. №2. Buenos Aires.

FIGUEIRA, Ricardo (1983a) "Del barro al ladrillo" In: Buenos Aires Historia de Cuatro Siglos (pp.102-119) Abril, Buenos Aires.

FIGUEIRA, Ricardo (1983b) "La Gran Aldea" In: Buenos Aires Historia de Cuatro Siglos (pp.285-303)

FINQUELIEVICH, Susana et E.LAURELLI (1990) "Innovación Tecnológica y reestructuración desigual del territorio: Países desarrollados-América 
Latina" In: Revista Interamericana de Planificación Vol XXIII-N8 9 (pp.191-212) SIAP, Guatemala.

GILLE, Bertrand (1978) Histoire des techniques. Gallimard, Pléiade. Paris

GIBERTI, Horacio (1981) Historia Económica de la Ganadería Argentina. (Chapitre VI) Solar, Buenos Aires.

GODET, Michel (1985) "Attention au mirage technologique" In: Les Cahiers Français...op.cit. Paris.

GOLD,D.,C.LO et E.O.WRIGHT (1985) "Recientes desarrollos en la teoría del Estado capitalista".(pp.171-205) In: Capitalismo y Estado. Revolución, Madrid.

GUEDON, Jean-Claude (1985). "Ce que nous apprend l'histoire des techniques" In: Les Cahiers Français....op cit. Paris

GUTMAN, Pablo, G.GUTMAN et G.DASCAL (1987) El campo en la ciudad. La producción agrícola en el Gran Buenos Aires. Sèrie Informes №6. CEUR, Buenos Aires.

HARVEY, David (1983) Teorías, leyes y modelos en Geografía (Explanation in Geography:1969) Chapitre XIV. Alianza Editorial, Madrid.

IGLESIAS de CUELLO, Alicia et G.SILVA (1986) "El sistema de comercialización frutihortícola en el Area Metropolitana" (pp.96-113) In: Aportes para el estudio del espacio socio-económico Volume I. El Coloquio, Buenos Aires.

KATZ, Jorge (1989) "Reestructuración industrial y teoría del crecimiento económico" In: Industria, Estado y Sociedad. La reestructuración industrial en América Latina y Europa (pp.17-28) Eural. Nueva Sociedad, Caracas.

KISHIMOTO, Nobuyasu et K.KOSHIRO (1992) Preliminary Study on a Master Plan for the La Plata Port. Municipality of La Plata. La Plata.

LAURELLI, Elsa et H.BOZZANO (1991) Nuevos escenarios tecnológicos y territorio. Los Polos Informático y Petroquímico de Berisso y Ensenada. In: Revista Interamericana de Planificación. Volume XXIV-Nº92. SIAP, Guatemala.

LAURELLI, Elsa et H.BOZZANO (1992) "Reestructuración productiva y Periferia. Territorios comprometidos. El caso de la microrregión de 
Ing.Allan-El Pato-El Peligro en la R.M.B.A.". CREDAL-Université Paris III (inédit)

LAURELLI, Elsa et J.LINDENBOIM (1992) Reestructuración de los mercados mundiales y transformación del territorio. Serie Cuadernos No30. CEUR, Buenos Aires.

LINDENBOIM, Javier (1987) Desarrollo regional y leyes promocionales. Serie Informes de Investigación N5. CEUR, Buenos Aires.

LYNCH, John (1983) "El crecimiento del comercio" In: Buenos Aires Historia de Cuatro Siglos.... (pp.187-205)

MARGER, Pierre Louis (1985) Nécessité d'une culture industrielle. In: Les Cahiers Français. $\mathrm{N} \circ 223$ Mutations technologiques et formations. La Documentation Française. Paris.

MASELIS, Analía (1982) "Puerto La Plata" (pp.196-212) In: La Plata una obra de arte. Posban \& Saltzmann, Buenos Aires.

McAUSLAN (1987) Tierra urbana y vivienda. Las opciones de los pobres. Earthscan, Nottingham.

MINSBURG, Naum (1987) Capitales extranjeros y grupos dominantes argentinos (análisis histórico y contemporáneo). Biblioteca Política Argentina $N^{\circ}$ 197. Centro Editor de América Latina, Buenos Aires.

MUNICIPALIDAD DE BERAZATEGUI (1989) Los usos del suelo y la subdivisión de la tierra en el Partido de Berazategui. Accord Departamento de Geografía,UNLP-Municipalidad Berazategui. La Plata.

ORTIZ, Ricardo (1978) Historia Económica de la Argentina (Quatrième Section) Plus Ultra, Buenos Aires.

PEET, Richard (1986) International capitalism and industria restructuring. Chapitre 2. Allen \& Unwin, Boston.

PEREZ BARRERO, María D.(1987) "Las Transformaciones socioeconómicas del Area Metropolitana. Reconversión industrial ) empleo. 1960-1987". Volume 1. CFI, Consejo Federal de Inversiones CFI Buenos Aires.

PERRIN, Jean-Claude (1990) "Organisation industrielle: La composant territoriale" In: Revue d'Economie Industrielle. N51 (pp.276-303) 
PRADA, Miriam et C.LILIU (1982) Estructura industrial del aglomerado platense. In: Revista de la Universidad N²9 (pp.81-94) UNLP, La Plata.

PROVINCIA DE BUENOS AIRES (1979) Estudio de la radicación industrial en la Prov.de Buenos Aires. Volume I. Secretaría de Planeamiento y Desarrollo, La Plata.

PROVINCIA DE BUENOS AIRES(1990) Polo Tecnológico Industrial de Berisso. Primera Etapa. Subsecretaría de Informática y Telecomunicaciones. La Plata.

PROVINCIA DE BUENOS AIRES (1990) Ley de Promoción Industrial 10547. Resolución 382. La Plata.

RAFFESTIN, Claude (1978) Pour une geographie du pouvoir. Paris.

RAFFESTIN, Claude et A.TURCO (1984) "Espace et pouvoir" In: Les concepts de la Géographie Humaine. Masson, Paris.

RANDLE, Patricio et N.GUREVITZ (1979) Atlas Histórico de la Pampa Anterior. Oikos Tau, Buenos Aires.

RECCHINI de LATTES, Zulma (1983) "La Población: Crecimiento explosivo y desaceleración, 1855-1980" In: Buenos Aires Historia de Cuatro Siglos (pp.241-254). Abril, Buenos Aires.

REVEL-MOUROZ,Jean (coord) (1989) Pouvoir local, régionalismes, décentralisation. Enjeux territoriaux et territorialité en Amérique Latine. IHEAL, Travaux et Memoires No47. Paris.

SANTAMARIA, Daniel (1983) "La Población: Estancamiento y expansión, 1580-1855" In: Buenos Aires Historia de Cuatro Siglos. Abril, Buenos Aires

SARRAILH, Eduardo (1983) "Lámparas y adoquines" In Buenos Aires Historia de Cuatro Siglos.(pp.403-420) Abril, Buenos Aires

SCHVARZER, Jorge (1983) "La implantación industrial" In: Buenos Aires Historia de Cuatro Siglos.(pp.223-239) Abril, Buenos Aires

SCOBIE, James et A.RAVINA de LUZZI (1983) "El centro, los barrios y el suburbio" In: Buenos Aires Historia de Cuatro Siglos.(pp.173-190) Abril, Buenos Aires. 
SIDOTTI, Juan, Y.SAFAR et R.AGUERO (1972) "Evolución en la localización industrial de Ensenada" In Semana de Geografía GAEA, Buenos Aires.

TADEO, Nidia (1991) La actividad florícola en el Sureste de la Región Metropolitana de Buenos Aires. Una producción en retroceso ?. Departamento de Geografía, UNLP. La Plata

TOPALOV, Christian (1979) La urbanización capitalista-Elementos para su análisis. Edicol, México.

TORRES, Horacio (1978) "Evolución de los procesos de estructuración espacial urbana. El caso de Buenos Aires" In: Revista Desarrollo Económico. Buenos Aires.

URBANSKI, Horacio (1982) "Berisso y el desarrollo del Partido de La Plata" In: La Plata, Ciudad Milagro. (pp.651-657)

VELUT, Sébastien (1990) Stratégies Industrielles à La Plata. Paris

YUJNOVSKY, Oscar (1983) "Del conventillo a la villa miseria" In: Buenos Aires Historia de Cuatro Siglos (pp451-487) 


\section{STATISTIQUES}

ADMINISTRACION GENERAL DE PUERTOS (1991) Anuario Puertos Argentinos-1990. Buenos Aires.

BOZZANO, H., M.A.GONZALEZ et G.PEIRANO (1990) "Evolución de la subdivisión de la tierra en los Partidos de La Plata, Ensenada y Berisso 1948-1989" La Plata (inédit)

DIRECCION GENERAL DE ESTADISTICA (1990) Censo Nacionai Agropecuario 1988-Provincia de Buenos Aires. Chapitres I et II. La Plata.

DIRECCION GENERAL DE ESTADISTICA (1991) Censo'91-Provincia dt Buenos Aires-Viviendas, Hogares y Población por Partidos y Localidades. Pcia.de Bs.As. La Plata.

INDEC (1992) Censo Nacional de Población y Vivienda 1991-Resultado: Provisionales. Instituto Nacional de Estadística y Censos, Buenos Aires.

INDEC (1972) Censo Nacional Agropecuario 1969-Datos de relevamiento agrícola. Instituto....., Buenos Aires.

INDEC (1982) Censo Nacional de Población y Vivienda 1980-Resultado. Provisionales. Instituto....., Buenos Aires

INDEC (1986) Censo Nacional Económico 1985-Resultado. Provisionales. Instituto..., Buenos Aires.

REPUBLICA ARGENTINA (1919) Tercer Censo Nacional-1914. Tomo V Explotaciones Agropecuarias. Buenos Aires. 


\section{CARTOGRAPHIE}

BOZZANO, Horacio et Patricia PINTOS (1989) Usos del suelo por parcela rural y manzana 1984 en La Plata, Ensenada, Berisso, Berazategui y Florencio Varela" Echelle 1:25.000. La Plata (inédit)

DIRECCION DE GEODESIA (1962) "Carta Parcelaria Rural de la Provincia de Buenos Aires" Echelles 1:400.000 et 1:200.000. Ministerio de Obras Públicas, La Plata.

DIRECCION DE GEODESIA (1973) Carte Topographique et Parcellaire "Villa Elisa". Feuille 3557-13-4. Echelle 1:25.000. Ministerio de Obras Públicas, Pcia de Bs As, La Plata.

DIRECCION DE GEODESIA (1974) Carte Topographique et Parcellaire "La Plata" Feuille 3557-14-3 et 14-1. Echelle 1:25.000. Ministerio de Obras Publicas. Pcia de bs As. La Plata.

DIRECCION DE GEODESIA (1980) Buenos Aires y Alrededores. Echelle 1:100.000. Ministerio de Obras Públicas Provincial. La Plata.

INSTITUTO GEOGRAFICO MILITAR ARGENTINO (1951) Carte Topographique "Villa Elisa"-Feuille 3557-13-4. Echelle 1:50.000. IGM, Buenos Aires.

INSTITUTO GEOGRAFICO MILITAR ARGENTINO (1951) Carte Topographique "La Plata"-Feuille 3557-14-3 et 14-1. Echelle 1:50.000. IGM, Buenos Aires.

MAPA RURAL (1988) Partido de La Plata. Echelle 1:105.000. Editorial Mapa Rural, La Plata.

VITAL, Adolfo et E.ROUX (s/d) "Nuevo Plano Catastral de los Alrrededores de Buenos Aires" Echelle 1:120.000. Date approximative 1915-1920. Publié par Hugo Bonvicini. 


\section{PRESSES}

PGM-Petroquímica General Mosconi: "Revista Temas" (1982-1987) No25 à No 37. PGM SAIyC, Buenos Aires

SAFRAR-Peugeot, Revista Peugeot

SHELL. "Petrokén, una carrera de obstáculos"(pp.6-7) In: Revue "Nosotros Shell" No81. Año VIII. Buenos Aires.

CIR2 (1992) Centro Industrial Ruta 2 (anciennement-Peugeot)

MAGAZINE LA PLATA. "La historia de Peugeot" №5. 1992. La Plata.

SIN LIMITES. "La ex-Peugeot levanta sus cortinas" El Pato, Berazategui. 1992

TRES LIMITES. Cooperativa Tres Límites Unidos. El Pato Berazategui. Plusieurs numéros(1987-1992)

-"LA NACION", Buenos Aires: 8 Octobre 1990; 23 Décembre 1990; 9 Mars 1991; 10 Mai 1991; 17 Juin 1991; 23 Juin 1991; 28 Juin 1992; 26 Juillet 1992; 16 Août 1992; 30 Août 1992; 6 Septembre 1992

-"CLARIN", Buenos Aires: 18 Mars 1983; 27 Juin 1991; 13 Octobre 1991; 14 Août 1992

-"EL DIA", La Plata: 26 Août 1980; 22 Février 1981; 6 Novembre 1981; 4 Mars 1984; 24 Novembre 1986; 27 Juin 1988; 27 Juin 1990; 26 Août 1990; 2 Décembre 1990; 22 Mai 1991; 9 Juin 1991; 16 Juin 1991; 20 Juin 1992; 29 Juin 1992; 20 Juillet 1992; 14 Août 1992; 16 Aout 1992; 26 Août 1992; 31 Août 1992; 1 Septembre 1992

-"PAGINA 12", Buenos Aires: 7 Juillet 1990 


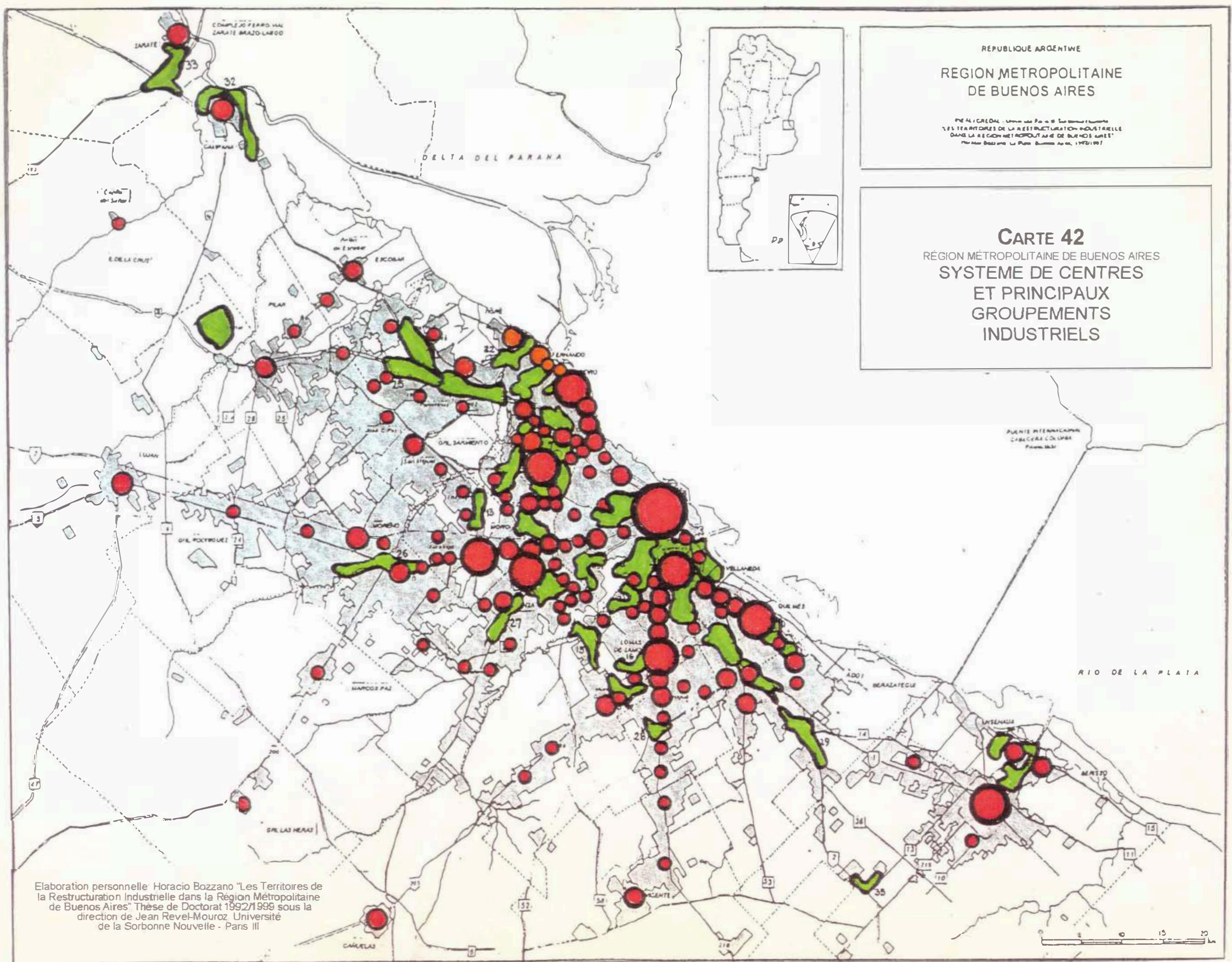




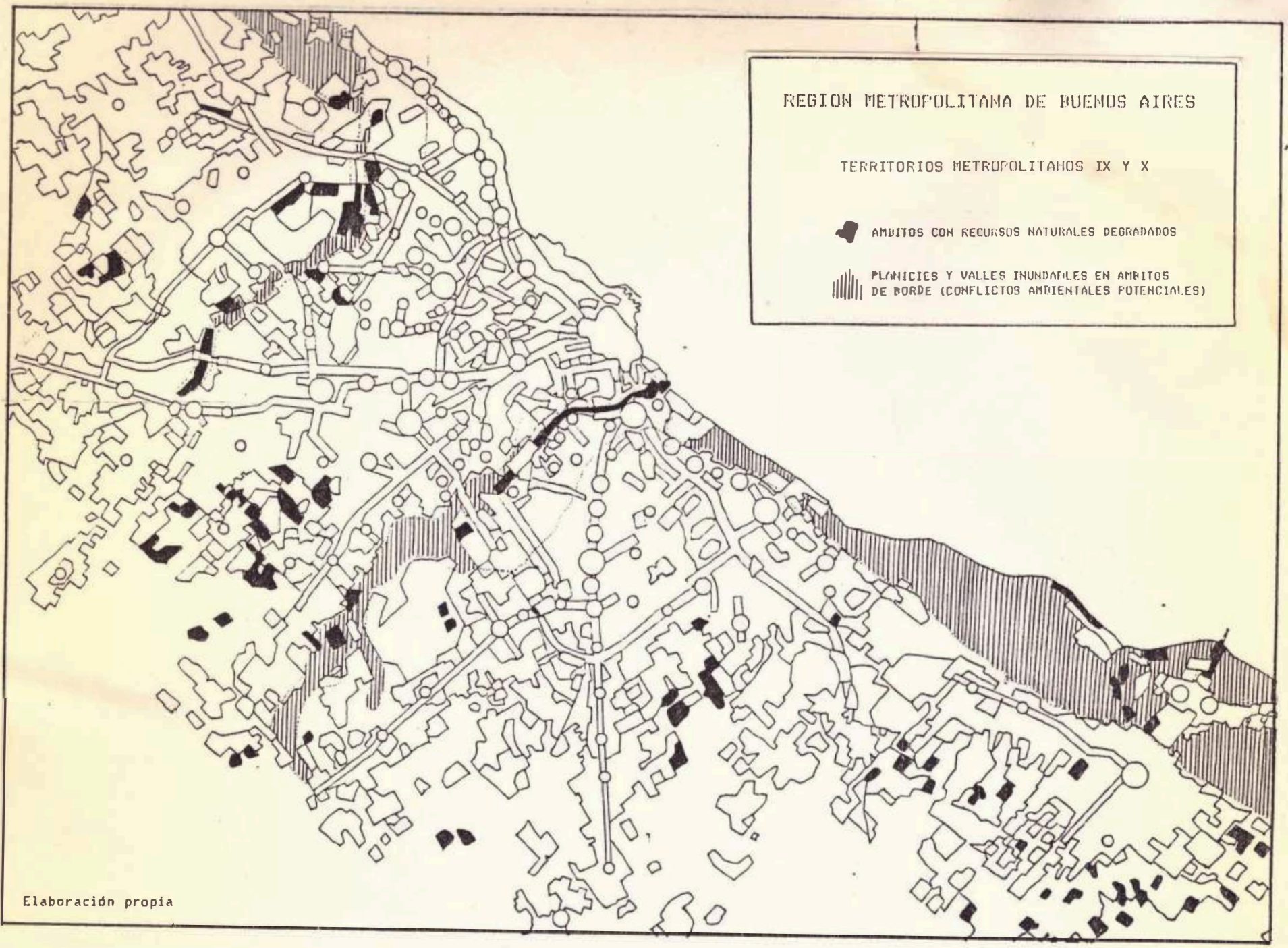




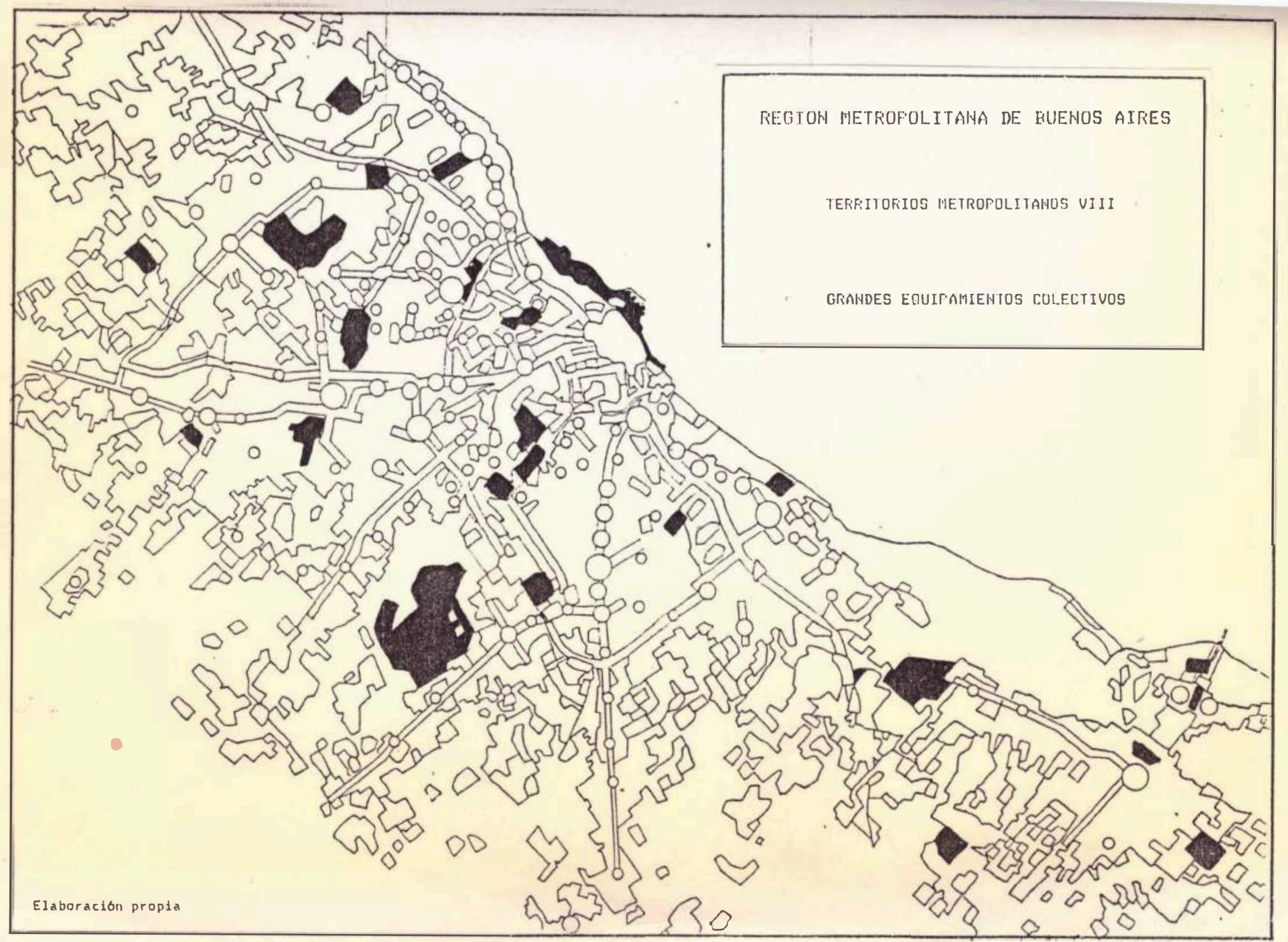




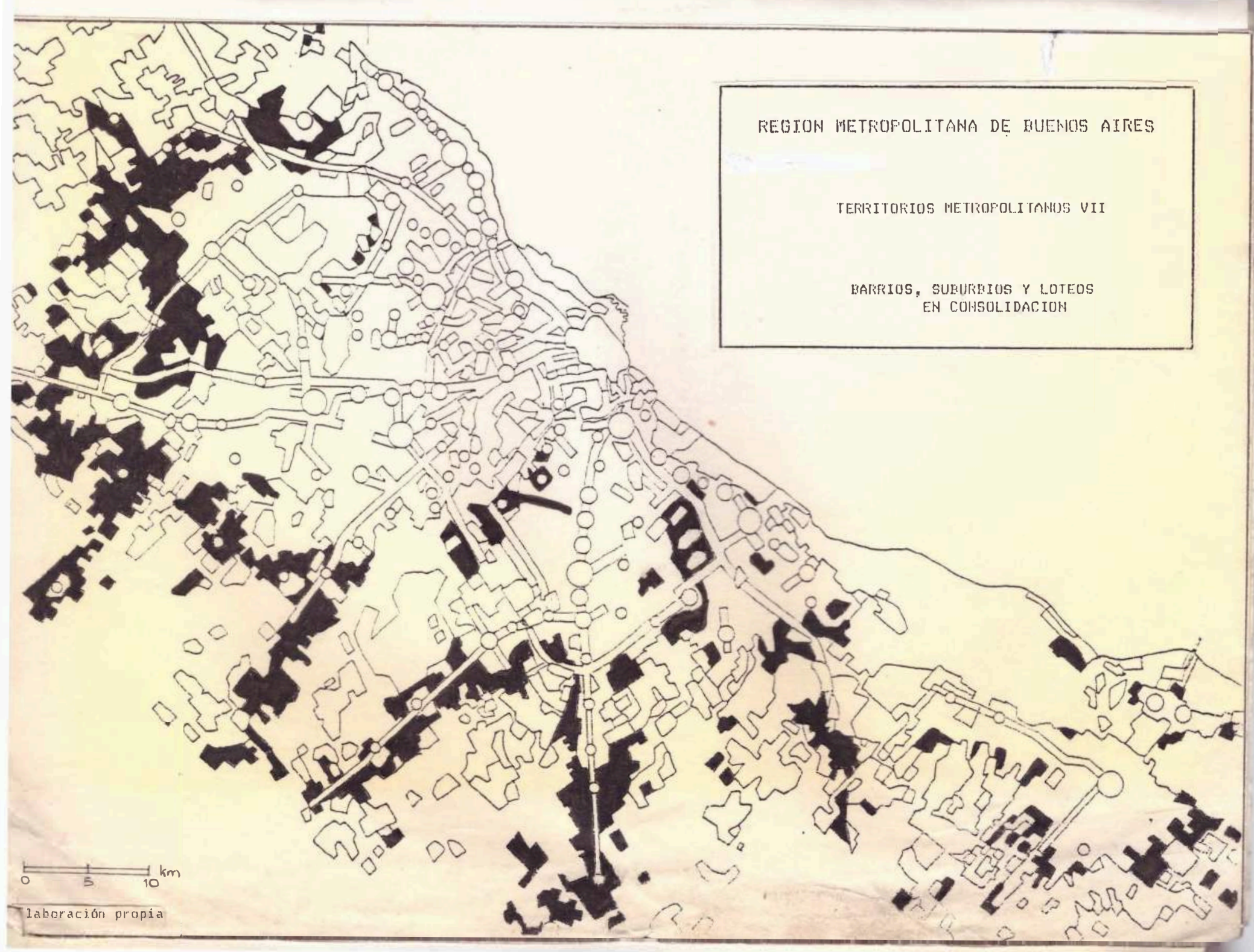




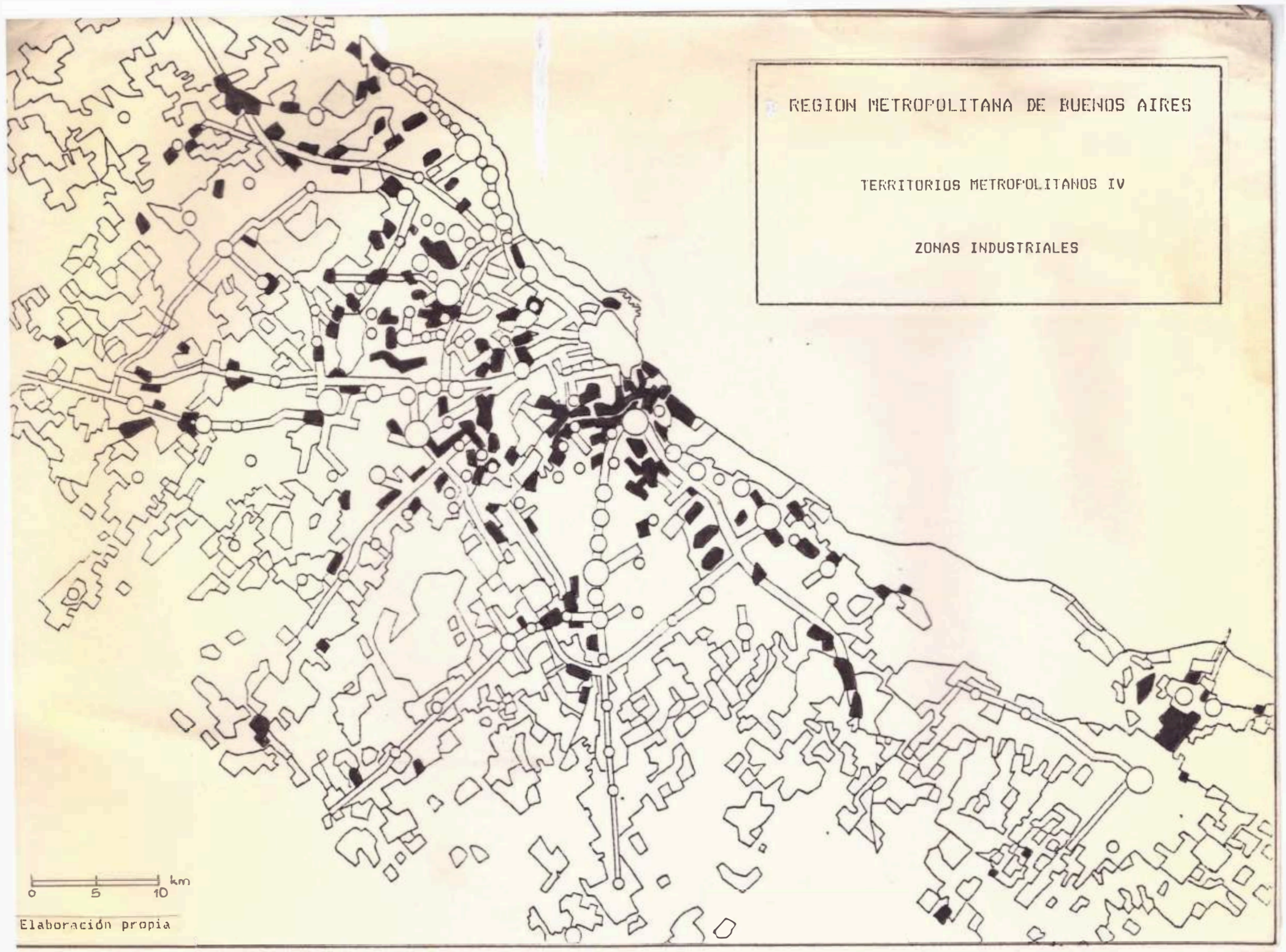




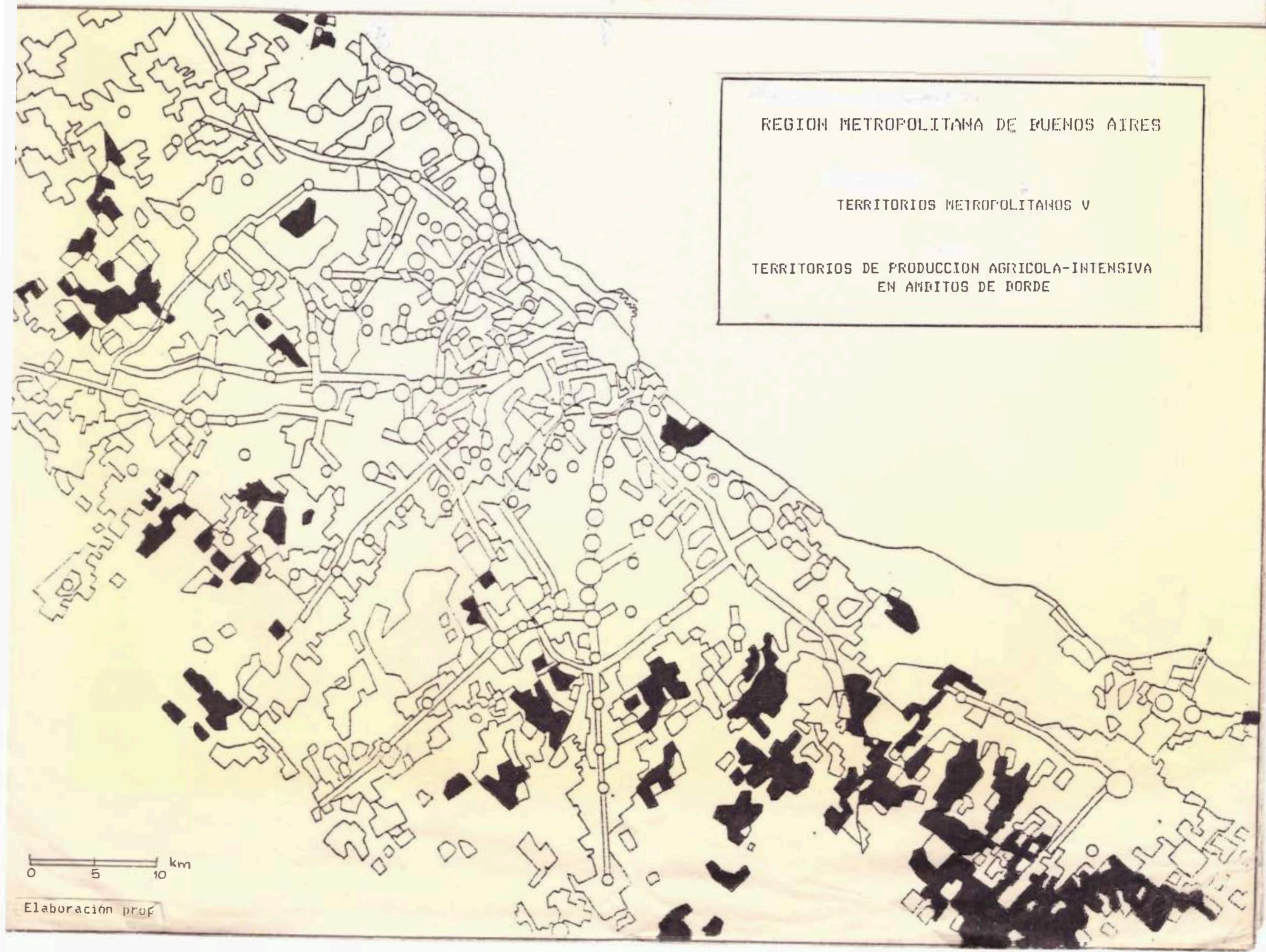




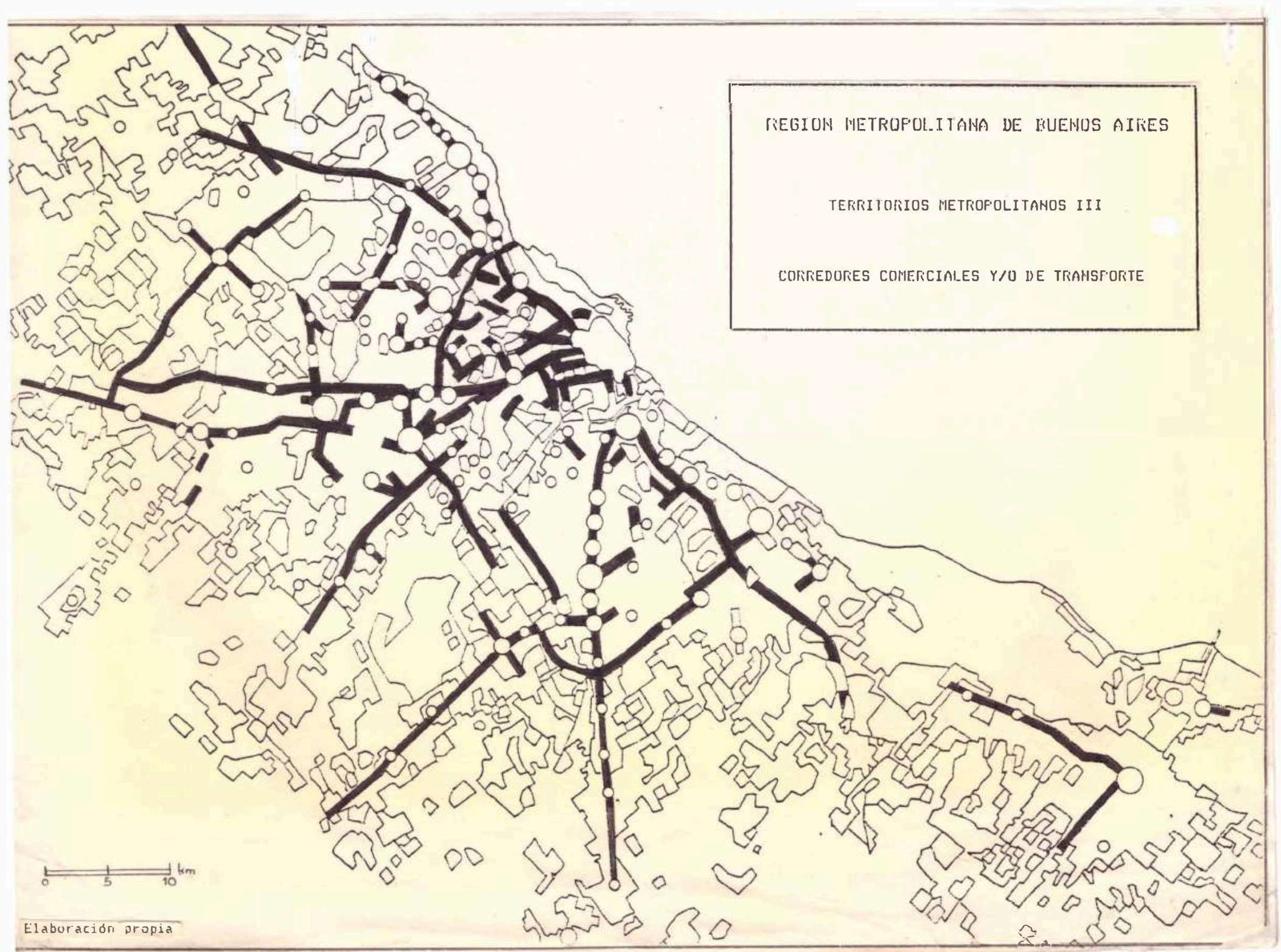

